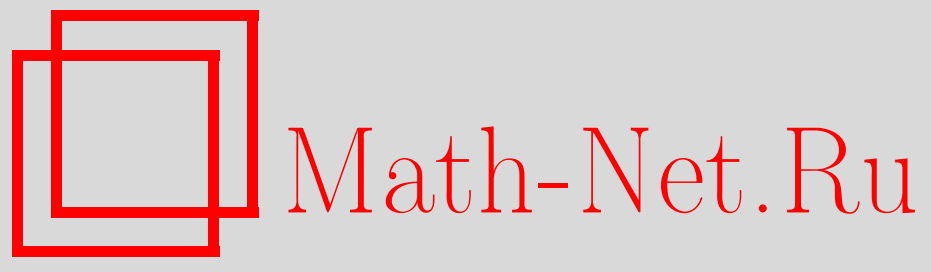

А. В. Арутюнов, Гладкие анормальные задачи теории экстремума и анализа, УМН, 2012, том 67, выпуск 3, 362

DOI: https://doi.org/10.4213/rm9478

Использование Общероссийского математического портала Math-Net.Ru подразумевает, что вы прочитали и согласны с пользовательским соглашением http: //www . mathnet.ru/rus/agreement

Параметры загрузки:

IP : 54.147 .182 .235

26 апреля 2023 г., 17:19:28

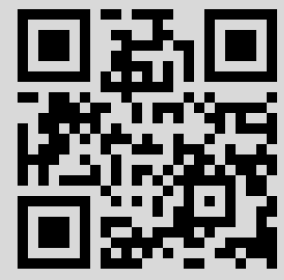




\title{
Гладкие анормальные задачи теории экстремума и анализа
}

\author{
А. В. Арутюнов
}

Работа содержит обзор результатов, связанных с теоремой об обратной функции, а также необходимых и достаточных условий экстремума первого и второго порядка для гладких экстремальных задач с ограничениями. Главным отличием приводимых результатов от классических является то, что они справедливы и содержательны без априорных предположений нормальности.

Библиография: 48 названий.

Ключевые слова: анормальная точка, принцип Лагранжа, необходимые и достаточные условия экстремума второго порядка, теорема об обратной функции, 2-регулярность, 2-нормальность, квадратичное отображение.

\section{СОДЕРЖАНИЕ}

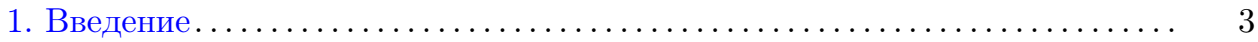

2. Необходимые условия экстремума второго порядка. Индексный подход 6

3. Необходимые условия экстремума первого и второго порядка. 2-регу-

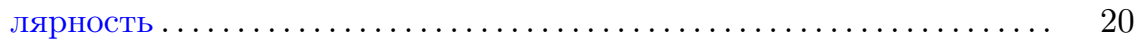

4. Достаточные условия экстремума второго порядка .............. 27

5. Квадратичные отображения и квадратичные задачи .............. 34

6. Теоремы об обратной функции ........................... 45

7. Необходимые условия второго порядка в задачах оптимального управления ....................................... 50

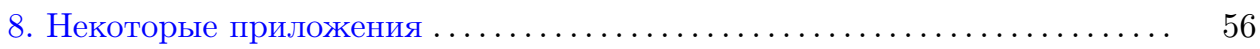

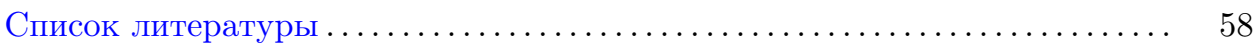

\section{1. Введение}

Исследование нелинейных экстремальных задач с ограничениями (задач на условный экстремум) основано на принципе Лагранжа, предложенном Ж. Лагранжем в конце XVIII века. Строгое обоснование принципа Лагранжа для широкого класса задач потребовало серьезных усилий многих математиков и было в основном закончено во второй половине XX века (см. [1] и библиографию

Работа выполнена при поддержке РФФИ (грант № 12-01-00427).

(C) А. В. Арутюнов, 2012 
там же), что в принципе ознаменовало окончание классического этапа развития теории. Однако, как мы поясним ниже, необходимые условия первого порядка в форме принципа Лагранжа содержательны лишь в предположении выполнения условий невырожденности (нормальности) ограничений, а в анормальном случае принцип Лагранжа не информативен.

Родственной тематикой являются теоремы об обратной или, более общо, о неявной функции. Они дают важнейший инструмент исследования нелинейных отображений и имеют огромное значение для теории и приложений. Первые теоремы об обратной функции появились во второй половине XIX века, а к концу XX века их обобщили на максимально общую ситуацию, включая негладкие отображения. Однако эти результаты были получены в предположении невырожденности (нормальности) рассматриваемого отображения, что в гладком случае означает сюръективность линейного оператора, определяемого его производной в рассматриваемой точке. Итак, к концу прошлого века в основном был завершен классический этап развития этой теории (см., например, [2]). На примере двух описанных задач поясним подробнее, что же такое анормальность.

Теория экстремальных задач. Пусть $X$ - векторное пространство. Рассмотрим задачу минимизации с ограничениями:

$$
f_{0}(x) \rightarrow \min , \quad F(x)=0 .
$$

Здесь $F: X \rightarrow Y=\mathbb{R}^{k}$ - заданное отображение, а минимум заданной функции $f_{0}: X \rightarrow \mathbb{R}$ ищется на допустимом множестве $M=\{x \in X: F(x)=0\}$. Для простоты вначале предположим, что $X$ - банахово пространство (можно даже считать, что $X=\mathbb{R}^{n}$ ), а $f_{0}$ и $F$ дважды непрерывно дифференцируемы в некоторой окрестности точки $x_{0},{ }^{1}$ являющейся локальным минимумом задачи (1.1). Тогда в точке $x_{0}$ выполнен принцип Лагранжа. Для его формулировки введем в рассмотрение функцию Лагранжа

$$
L(x, \lambda)=\lambda^{0} f_{0}(x)+\left\langle y^{*}, F(x)\right\rangle ; \quad \lambda=\left(\lambda^{0}, y^{*}\right), \quad \lambda^{0} \in \mathbb{R}, \quad y^{*} \in Y^{*},
$$

где $(k+1)$-мерный вектор $\lambda=\left(\lambda^{0}, y^{*}\right)$ и его компоненты называются множителями Лагранжа, а угловые скобки, как обычно, означают скалярное умножение ${ }^{2}$.

ТЕоремА 1.1 (принцип Лагранжа). Пусть $x_{0}$ является точкой локалъного минимума задачи (1.1). Тогда существует такой множитель Лагранжа $\lambda$, чmo

$$
\frac{\partial L}{\partial x}\left(x_{0}, \lambda\right)=0, \quad \lambda^{0} \geqslant 0, \quad \lambda \neq 0 .
$$

Принцип Лагранжа дает необходимые условия экстремума первого порядка и хорошо известен (см. [1], [3] и т. д.).

\footnotetext{
${ }^{1}$ Во введении для простоты накладываются избыточные предположения гладкости

${ }^{2}$ Множитель Лагранжа $y^{*}$ берут из пространства $Y^{*}$, топологически сопряженного к $Y$. Однако там, где $Y$ предполагается конечномерным арифметическим пространством, мы будем отождествлять $Y$ с его сопряженным и считать множитель Лагранжа $y^{*}$ элементом пространства $Y$.
} 
Рассмотрим два случая. Пусть вначале точка $x_{0}$ является нормальной, т. е. $\operatorname{im} F^{\prime}\left(x_{0}\right)=Y$. (В отечественной литературе в этом случае часто говорят, что в точке $x_{0}$ выполнено условие Люстерника. Кроме того, наряду с термином "нормальная" точка, часто употребляют также термины "невырожденная" или "регулярная" точка.) Итак, если точка минимума $x_{0}$ нормальна, то в силу $(1.3)$ $\lambda^{0}>0$ и, следовательно, учитывая положительную однородность соотношений (1.3) по переменной $\lambda$, можно считать, что $\lambda^{0}=1$. При этом существует единственный множитель Лагранжа, имеющий вид $\lambda=\left(1, y^{*}\right)$, причем для него выполняются классические необходимые условия второго порядка

$$
\frac{\partial^{2} L}{\partial x^{2}}\left(x_{0}, \lambda\right)[x, x] \geqslant 0 \quad \forall x \in X: F^{\prime}\left(x_{0}\right) x=0
$$

(см. [3; § 3.4, с. 287]). Здесь квадратные скобки обозначают действие билинейной формы.

Рассмотрим второй случай: точка $x_{0}$ анормальна, т. е. $\operatorname{im} F^{\prime}\left(x_{0}\right) \neq Y$. Тогда в ней принцип Лагранжа (1.3) выполняется при $\lambda^{0}=0$ и произвольном $y^{*} \neq 0$, принадлежащем ядру сопряженного оператора $\operatorname{ker} F^{\prime}\left(x_{0}\right)^{*}$, которое является ненулевым, поскольку im $F^{\prime}\left(x_{0}\right) \neq Y$. Таким образом, в любой анормальной точке принцип Лагранжа автоматически выполняется независимо от минимизируемого функционала $f_{0}$ и является лишь прямым следствием определения анормальности. Поэтому принцип Лагранжа бесполезен при исследовании анормальной точки на экстремум. Что же касается классических необходимых условий второго порядка (1.4), то в анормальной точке минимума они могут нарушаться. Вот простой двумерный пример, подтверждающий это:

$$
\begin{gathered}
X=\mathbb{R}^{2}, \quad f_{0}(x)=-|x|^{2} \rightarrow \min , \\
F_{1}(x)=x_{1}^{2}-x_{2}^{2}=0, \quad F_{2}(x)=x_{1} x_{2}=0,
\end{gathered}
$$

где $x=\left(x_{1}, x_{2}\right) \in \mathbb{R}^{2}$. В этой задаче ограничениям удовлетворяет единственная точка $x_{0}=0$, естественно являющаяся точкой минимума. Однако в ней условия (1.4) не выполняются ни при каком удовлетворяющем (1.3) множителе Лагранжа $\lambda$. Итак, в анормальной точке принцип Лагранжа неинформативен, а классические необходимые условия второго порядка могут нарушаться. Таким образом, возникает проблема нахождения содержательных необходимых условий минимума в задаче (1.1) без априорных предположений нормальности исследуемой точки.

Теорема об обратной функции. Пусть отображение $F: X \rightarrow Y$ непрерывно дифференцируемо в окрестности точки $x_{0} \in X$ и $y_{0}=F\left(x_{0}\right)$. Спрашивается, существует ли такая окрестность $V$ точки $y_{0}$, что для всех $y \in V$ уравнение

$$
F(x)=y
$$

имеет такое решение $x(y)$, что $x\left(y_{0}\right)=x_{0}$ и отображение $x(\cdot)$ непрерывно в точке $y_{0}$, или даже более того, непрерывно на всей окрестности $V$. Если точка $x_{0}$ нормальна, то классическая теорема об обратной функции дает на этот вопрос положительный ответ, причем отображение $x(\cdot)$ можно выбрать непрерывно 
дифференцируемым. Если же точка $x_{0}$ анормальна, то это уже не так. Например, скалярное уравнение $x_{1}^{2}+x_{2}^{2}=y$, рассматриваемое в окрестности нуля, для $y<0$ не имеет решений, а уравнение $x_{1}^{2}-x_{2}^{2}=y$ имеет бесконечно много непрерывных решений, для которых $x(0)=0$, но все они в нуле не дифференцируемы и даже, более того, не удовлетворяют условию Липшица. Итак, возникает проблема нахождения условий, более слабых, чем нормальность, которые гарантируют существование решения $x(\cdot)$ уравнения (1.6) с нужными свойствами.

Обсуждению этих двух, а также других, близких к ним, задач и посвящен настоящий обзор. При этом основное внимание акцентируется на последних достижениях, а история вопроса и ранние работы, усиленные впоследствие, как правило, оставлены в стороне. Следующие разделы 2 и 3 содержат необходимые условия экстремума первого и второго порядка для задач с различными типами ограничений. Отличительной чертой этих результатов является то, что они получены и содержательны без априорных предположений нормальности, а в нормальном случае превращаются в классические условия. Эти два раздела отличаются тем, что они содержат два различных подхода: в первом из них результаты получены в терминах индексов вторых производных классической функции Лагранжа $L$, а в разделе 3 исследования основаны на обобщенной функции Лагранжа $\mathscr{L}_{\mathscr{A}}$. Раздел 4 содержит достаточные условия второго порядка. В нем наряду с классическими приведены достаточные условия для задач с незамкнутым образом и достаточные условия в терминах функции $\mathscr{L}_{\mathscr{A}}$ для тех классов анормальных задач, в которых классические достаточные условия автоматически вырождаются. В разделе 5 изучаются квадратичные задачи, дающие типичный пример класса анормальных задач, а также получены некоторые результаты по теории квадратичных отображений. Раздел 6 содержит различные варианты теорем об обратной и неявной функции. Так же как и указанные выше результаты, они получены и содержательны без априорных предположений нормальности, а в нормальном случае превращаются в классические теоремы. В разделе 7 в качестве приложения абстрактной теории, изложенной в разделах 2 и 3 , получены необходимые условия оптимальности второго порядка для различных задач оптимального управления. Раздел 8 посвящен приложению изложенных абстрактных результатов к теории бифуркаций, теории чувствительности, управляемости управляемых динамических систем и теории квадратичных отображений.

\section{2. Необходимые условия экстремума второго порядка. Индексный подход}

Экстремальная задача с ограничениями типа равенств и неравенств. Пусть по-прежнему $X$ - векторное пространство и $Y=\mathbb{R}^{k}$. Заданы функции $f_{i}: X \rightarrow \mathbb{R}, i=\overline{0, l}$, и отображение $f: X \rightarrow Y$. Рассмотрим экстремальную задачу с ограничениями типа равенств и неравенств

$$
f_{0}(x) \rightarrow \min , \quad f_{j}(x) \leqslant 0, \quad j=\overline{1, l}, \quad f(x)=0 .
$$


Пусть точка $x_{0} \in X$ удовлетворяет ограничениям задачи (2.1). В дальнейшем для простоты будем считать, что все индексы, отвечающие ограничениям типа неравенств, активны, т. е. $f_{j}\left(x_{0}\right)=0, \quad j=\overline{1, l}$. Это не нарушает общности, так как при локальных рассмотрениях, которыми мы занимаемся, ограничения $f_{j}: f_{j}\left(x_{0}\right)<0$ можно опустить.

Заданные функции $f_{j}$ и отображение $f: X \rightarrow Y$ будем предполагать гладкими в следующем смысле. Обозначим через $\mathscr{M}$ множество всевозможных конечномерных подпространств $M \subseteq X$, каждое из которых наделим единственной отделимой векторной топологией. Через $\|\cdot\|_{M}$ будем обозначать какую-нибудь из (эквивалентных) норм, порождающих в $M$ эту топологию. В векторном пространстве $X$ введем так называемую конечную топологию. В ней открытыми считаются те и только те множества, пересечение которых с любым $M \in \mathscr{M}$ открыто. Если пространство $X$ бесконечномерно, то оно, будучи наделенным конечной топологией, вообще говоря, не является векторным топологическим пространством, так как в нем сложение, как правило, разрывно. С другой стороны, конечная топология сильнее всех топологий, превращающих $X$ в векторное топологическое пространство. При этом локальный относительно конечной топологии минимум является слабейшим среди всех рассматриваемых типов минимума.

Относительно отображения $f$ будем предполагать, что оно дважды непрерывно дифференцируемо в окрестности точки $x_{0}$ относительно конечной топологии $\tau$. Последнее означает, что для произвольного конечномерного линейного подпространства $M$ сужение $f$ на $M$ дважды непрерывно дифференцируемо в некоторой (зависящей от $M$ ) окрестности $x_{0}$. Следовательно, существуют линейный оператор $A: X \rightarrow Y$ и билинейное отображение $Q: X \times X \rightarrow Y$, а также отображение $\alpha: X \rightarrow \mathrm{Y}$ такие, что

$$
f(x)=f\left(x_{0}\right)+A\left(x-x_{0}\right)+\frac{1}{2} Q\left[x-x_{0}, x-x_{0}\right]+\alpha\left(x-x_{0}\right) \quad \forall x \in X
$$

и для произвольного конечномерного подпространства $M$ имеет место

$$
\frac{\left\|\alpha\left(x-x_{0}\right)\right\|}{\left\|x-x_{0}\right\|_{M}^{2}} \rightarrow 0, \quad x \rightarrow x_{0}, \quad x \in M .
$$

Отображения $A$ и $Q$ обозначаются через $f^{\prime}\left(x_{0}\right)$ (или $\left.\frac{\partial f}{\partial x}\left(x_{0}\right)\right)$ и $f^{\prime \prime}\left(x_{0}\right)$ (или $\left.\frac{\partial^{2} f}{\partial x^{2}}\left(x_{0}\right)\right)$ и называются первой и второй производными $f$. Аналогичные предположения считаются выполненными относительно всех функций $f_{j}$. Предположение гладкости относительно конечной топологии является слабейшим среди обычно рассматриваемых.

Важно отметить, что последующие результаты остаются содержательными и в случае, когда $X=\mathbb{R}^{n}$, a $f, f_{j}$ дважды непрерывно дифференцируемы в обычном смысле. Поэтому если не интересоваться бесконечномерной спецификой, то можно без ущерба для понимания общего замысла предполагать, что $X=\mathbb{R}^{n}$. 
Определим функцию Лагранжа для задачи (2.1):

$$
L(x, \lambda)=\sum_{j=0}^{l} \lambda^{j} f_{j}(x)+\left\langle y^{*}, f(x)\right\rangle ; \quad \lambda=\left(\lambda^{0}, \ldots, \lambda^{l}, y^{*}\right), \quad \lambda^{j} \in \mathbb{R}, \quad y^{*} \in Y^{*} .
$$

Рассмотрим множество нормированных множителей Лагранжа задачи (2.1), отвечающих точке $x_{0}$ в силу принципа Лагранжа:

$$
\Lambda\left(x_{0}\right)=\left\{\lambda: \frac{\partial L}{\partial x}\left(x_{0}, \lambda\right)=0, \lambda^{j} \geqslant 0 \forall j, \sum_{j=0}^{l} \lambda^{j}+\left|y^{*}\right|=1\right\} .
$$

(Отметим, что условия дополняющей нежесткости $\lambda^{j} f_{j}\left(x_{0}\right)=0, j=\overline{1, l}$, здесь опущены, так как в силу сделанного предположения $f_{j}\left(x_{0}\right)=0, j=\overline{1, l}$.)

Для целых неотрицательных $s$ введем в рассмотрение множества (возможно, некоторые из них пусты) $\Lambda_{s}\left(x_{0}\right)$, состоящие из тех множителей Лагранжа $\lambda \in \Lambda\left(x_{0}\right)$, для которых существует такое (зависящее от $\lambda$ ) линейное подпространство $\Pi \subseteq X$, что

$$
\operatorname{codim} \Pi \leqslant s ; \quad \Pi \subseteq \operatorname{ker} F^{\prime}\left(x_{0}\right) ; \quad \frac{\partial^{2} L}{\partial x^{2}}\left(x_{0}, \lambda\right)[x, x] \geqslant 0 \quad \forall x \in \Pi .
$$

Здесь и ниже отображение $F=\left(f_{1}, \ldots, f_{l}, f\right)$ действует из $X$ в $\mathbb{R}^{m}$ и

$$
m=k+l \text {. }
$$

Введем в рассмотрение конус

$$
\mathscr{K}\left(x_{0}\right)=\left\{h \in X:\left\langle f_{j}^{\prime}\left(x_{0}\right), h\right\rangle \leqslant 0, j=\overline{0, l} ; f^{\prime}\left(x_{0}\right) h=0\right\},
$$

называемый критическим. Он, очевидно, выпуклый и непустой $\left(0 \in \mathscr{K}\left(x_{0}\right)\right)$.

ТЕОРема 2.1 (необходимые условия второго порядка). Пусть в задаче (2.1) точка $x_{0}$ является локальным минимумом относительно конечной топологии. Тогда множество $\Lambda_{m}\left(x_{0}\right)$ непусто и, более того,

$$
\max _{\lambda \in \Lambda} \frac{\partial^{2} L}{\partial x^{2}}\left(x_{0}, \lambda\right)[x, x] \geqslant 0 \quad \forall x \in \mathscr{K}\left(x_{0}\right), \quad \Lambda=\Lambda_{m}\left(x_{0}\right) .
$$

Доказательство теоремы использует следующую конструкцию.

Оценка снизу верхнего топологического предела последовательности подпространств. Если $\left\{\Pi_{i}\right\}$ - последовательность подмножеств метрического пространства, то $\operatorname{Ls}\left\{\Pi_{i}\right\}$ обозначает ее верхний топологический предел, который состоит из всевозможных предельных точек последовательностей $\left\{x_{i}\right\}: x_{i} \in \Pi_{i} \forall i$.

Пусть $X$ - банахово пространство и $\left\{A_{i}\right\}_{i=1}^{\infty}-$ последовательность линейных непрерывных операторов, действующих из $X$ в $Y=\mathbb{R}^{k}$. Будем предполагать, что последовательность $\left\{A_{i}\right\}$ сходится по норме к линейному оператору $A: X \rightarrow Y$. Положим $M=\operatorname{Ls}\left\{\operatorname{ker} A_{i}\right\}$. Очевидно, что $M$ замкнуто, непусто $(0 \in M)$ и $M \subseteq \operatorname{ker} A$. Если оператор $A$ сюръективен, то $M=\operatorname{ker} A$. Если же $A$ сюръективным не является, то, вообще говоря, $M \neq \operatorname{ker} A$ и множество $M$ может даже не быть выпуклым. 
Tеорема 2.2. В Х существует такое замкнутое подпространство П, что $\operatorname{codim} \Pi \leqslant k ; \quad \Pi \subseteq \operatorname{Ls}\left\{\operatorname{ker} A_{i}\right\} ; \quad \Pi \subseteq \operatorname{ker} A$.

Справедливость этой теоремы непосредственно вытекает из следующего утверждения, имеющего и самостоятельное значение.

Теорема 2.3. Пусть $\left\{\Pi_{i}\right\}$ - последовательность замкнутых подпространств из $X$ и codim $\Pi_{i} \leqslant k \forall i$. Тогда существует такое замкнутое подпространство $\Pi \subseteq X$, что

$$
\operatorname{codim} \Pi \leqslant k ; \quad \Pi \subseteq \operatorname{Ls}\left\{\Pi_{i}\right\} .
$$

Из теоремы 2.3 вытекает замкнутость, а значит, и компактность множеств $\Lambda_{s}\left(x_{0}\right)$ при любом $s$ в случае, когда $X$ - банахово пространство, а отображения $f_{i}, f$ гладкие. Это важное свойство используется ниже. Отложим доказательство теоремы 2.3 до конца раздела.

Доказательство теоремы 2.1. Следуя [4]--[6], доказательство теоремы 2.1 проведем в три этапа. Вначале (этап I) в предположении конечномерности $X$ докажем непустоту $\Lambda_{m}\left(x_{0}\right)$. На этапе II докажем теорему для конечномерных $X$. Наконец, на этапе III докажем теорему в полной общности (т. е. избавимся от предположения $\operatorname{dim} X<\infty)$.

Этап I. Будем предполагать, что пространство $X$ конечномерно. Тогда, не теряя общности, можно считать, что $X=\mathbb{R}^{n}$. Выберем $\delta>0$ так, чтобы в задаче

$$
f_{0}(x) \rightarrow \min , \quad f_{j}(x) \leqslant 0, \quad j=\overline{1, l}, \quad f(x)=0, \quad\left|x-x_{0}\right| \leqslant \delta
$$

точка $x_{0}$ являлась минимумом. Снимем в этой задаче ограничения (все, кроме последнего) с помощью метода штрафов. Для каждого натурального $i$ положим

$$
\varphi_{i}(x)=f_{0}(x)+i\left(\sum_{j=1}^{l}\left(f_{j}(x)^{+}\right)^{4}+|f(x)|^{4}\right)+\left|x-x_{0}\right|^{4},
$$

где $a^{+}=\max (a, 0)$. Рассмотрим последовательность задач минимизации

$$
\varphi_{i}(x) \rightarrow \min , \quad\left|x-x_{0}\right| \leqslant \delta,
$$

называемых $i$-задачами. Решение в $i$-задаче существует в силу непрерывности $\varphi_{i}$ и компактности замкнутого шара в конечномерном пространстве $X$. Пусть $x_{i}$ - какое-нибудь из решений $i$-задачи.

Докажем, что $x_{i} \rightarrow x_{0}$. Действительно, учитывая конечномерность $X$ и переходя к подпоследовательности, добьемся того, что $x_{i} \rightarrow \bar{x}$. Покажем, что $\bar{x}=x_{0}$. Имеем

$$
\begin{aligned}
\varphi_{i}\left(x_{i}\right) \leqslant \varphi_{i} & \left(x_{0}\right)=f_{0}\left(x_{0}\right) \forall i \\
& \Rightarrow \lim _{i \rightarrow \infty} f_{j}\left(x_{i}\right) \leqslant 0, j=\overline{1, l}, f\left(x_{i}\right) \rightarrow 0 \\
& \Rightarrow f_{j}(\bar{x}) \leqslant 0, j=\overline{1, l}, f(\bar{x})=0 \\
& \Rightarrow f_{0}(\bar{x}) \geqslant f_{0}\left(x_{0}\right) .
\end{aligned}
$$


Кроме того, из первого неравенства имеем

$$
\begin{aligned}
& f_{0}\left(x_{i}\right)+\left|x_{i}-x_{0}\right|^{4} \leqslant f_{0}\left(x_{0}\right) \forall i \\
& \quad \Rightarrow f_{0}(\bar{x})+\left|\bar{x}-x_{0}\right|^{4} \leqslant f_{0}\left(x_{0}\right) \leqslant f_{0}(\bar{x}) \Rightarrow \bar{x}=x_{0} .
\end{aligned}
$$

Таким образом, доказано, что $x_{i} \rightarrow x_{0}$. Поэтому при всех больших $i$, которые мы только и будем рассматривать, имеет место неравенство $\left|x_{i}-x_{0}\right|<\delta$ и, значит, $i$-задача локально эквивалентна задаче минимизации гладкой функции без ограничений, и, значит, необходимые условия первого и второго порядка для нее заключаются в том, что

$$
\varphi_{i}^{\prime}\left(x_{i}\right)=0, \quad \varphi_{i}^{\prime \prime}\left(x_{i}\right)[x, x] \geqslant 0 \quad \forall x .
$$

Расшифровывая их, получаем

$$
\begin{gathered}
\frac{\partial L}{\partial x}\left(x_{i}, \lambda_{i}\right)+\kappa_{i} \cdot 4\left(x_{i}-x_{0}\right)\left|x_{i}-x_{0}\right|^{2}=0 \\
\frac{\partial^{2} L}{\partial x^{2}}\left(x_{i}, \lambda_{i}\right)[x, x]+12 \kappa_{i}\left(i \sum_{j=1}^{m}\left(f_{j}\left(x_{i}\right)^{+}\right)^{2}\left|\left\langle f_{j}^{\prime}\left(x_{i}\right), x\right\rangle\right|^{2}\right. \\
\left.+i\left|f\left(x_{i}\right)\right|^{2}\left|f^{\prime}\left(x_{i}\right) x\right|^{2}+1(i)|x|^{2}\right) \geqslant 0 \quad \forall x
\end{gathered}
$$

где $1(i) \rightarrow 0, i \rightarrow \infty$, а также $\lambda_{i}=\kappa_{i}\left(1, \bar{\lambda}_{i}^{1}, \ldots, \bar{\lambda}_{i}^{l}, \bar{y}_{i}^{*}\right)$,

$$
\begin{gathered}
\bar{\lambda}_{i}^{j}=4 i\left(f_{j}\left(x_{i}\right)^{+}\right)^{3}, \quad j=\overline{1, l}, \quad \bar{y}_{i}^{*}=4 i\left|f\left(x_{i}\right)\right|^{2} f\left(x_{i}\right), \\
\kappa_{i}=\left(1+\sum_{j=1}^{l}\left(\bar{\lambda}_{i}^{j}\right)^{2}+\left|\bar{y}_{i}^{*}\right|^{2}\right)^{-1 / 2} .
\end{gathered}
$$

По построению $\left|\lambda_{i}\right|=1, \bar{\lambda}_{i}^{j} \geqslant 0 \forall i, \forall j \leqslant l$. Поэтому после перехода к подпоследовательности и последующего предельного перехода в (2.6) имеем $\lambda_{i} \rightarrow$ $\lambda \in \Lambda\left(x_{0}\right)$.

Докажем существование подпространства П, удовлетворяющего (2.3) при $s=m$. Для этого рассмотрим линейные операторы $A_{i}=F^{\prime}\left(x_{i}\right)$. По теореме 2.2 существует подпространство $\Pi$, для которого $\Pi \subseteq \operatorname{ker} F^{\prime}\left(x_{0}\right), \Pi \subseteq \operatorname{Ls}\left\{\operatorname{ker} A_{i}\right\}$, $\operatorname{codim} \Pi \leqslant m$. Возьмем произвольный вектор $h \in \Pi$. В силу определения верхнего топологического предела существуют $h_{i} \in \operatorname{ker} F^{\prime}\left(x_{i}\right)$ такие, что после перехода к подпоследовательности $h_{i} \rightarrow h$. Подставляя в $(2.7) x=h_{i}$ и переходя к пределу при $i \rightarrow \infty$, получаем, что $\frac{\partial^{2} L}{\partial x^{2}}\left(x_{0}, \lambda\right)[h, h] \geqslant 0$. Отсюда, в силу произвольности $h \in \Pi$, имеем $\frac{\partial^{2} L}{\partial x^{2}}\left(x_{0}, \lambda\right)[x, x] \geqslant 0 \forall x \in \Pi$. Таким образом, доказано, что $\lambda \in \Lambda_{m}\left(x_{0}\right)$.

Этап II. Пусть по-прежнему $X=\mathbb{R}^{n}$. Докажем (2.4). Для удобства будем считать, что $x_{0}=0, f_{0}\left(x_{0}\right)=0$. Введем в рассмотрение функцию $\gamma$ :

$$
\gamma(\chi)=0 \quad \forall \chi \leqslant 1, \quad \gamma(\chi)=(\chi-1)^{4} \quad \forall \chi>1 .
$$


Зафиксируем произвольный единичный вектор $h \in \mathscr{K}\left(x_{0}\right)$, для которого $|h|=1$ (если, конечно, $\mathscr{K}\left(x_{0}\right) \neq\{0\}$ ). Для $\varepsilon=i^{-1}, i=1,2, \ldots$, рассмотрим задачу минимизации по переменной $(x, \chi) \in X \times \mathbb{R}$ :

$$
\begin{gathered}
f_{\varepsilon}(x, \chi) \rightarrow \min , \quad f_{j}(x)-\chi f_{j}(\varepsilon h) \leqslant 0, \quad j=\overline{1, l}, \\
f(x)-\chi f(\varepsilon h)=0, \quad \chi \geqslant 0, \quad|x| \leqslant \delta,
\end{gathered}
$$

называемую $\varepsilon$-задачей. Здесь $\delta$ определено выше и

$$
f_{\varepsilon}(x, \chi)=\tilde{f}_{0}(x)-\chi \tilde{f}_{0}(\varepsilon h)+\gamma(\chi), \quad \tilde{f}_{0}(x)=f_{0}(x)+|x|^{4} .
$$

При $\varepsilon<\delta$, которые только и будем рассматривать, решение $\varepsilon$-задачи существует, поскольку точка $x=\varepsilon h, \quad \chi=1$ удовлетворяет всем ее ограничениям, шар $\{x:|x| \leqslant \delta\}$ компактен и $\gamma(\chi) / \chi \rightarrow \infty$ при $\chi \rightarrow \infty$.

Покажем, что среди решений $\left(x_{\varepsilon}, \chi_{\varepsilon}\right) \varepsilon$-задачи существует такое, что $\chi_{\varepsilon}>0$. Действительно, пусть $\left(x_{\varepsilon}, 0\right)$ является ее решением. Покажем, что тогда $x_{\varepsilon}=0$. Действительно, $x_{\varepsilon}$ удовлетворяет всем ограничениям задачи $(2.1)$ и если $x_{\varepsilon} \neq 0$, то

$$
f_{\varepsilon}\left(x_{\varepsilon}, 0\right)=f_{0}\left(x_{\varepsilon}\right)+\left|x_{\varepsilon}\right|^{4} \geqslant f_{0}(0)+\left|x_{\varepsilon}\right|^{4}>0 \quad \text { и } \quad f_{\varepsilon}(\varepsilon h, 1)=0 \Rightarrow f_{\varepsilon}\left(x_{\varepsilon}, 0\right) \leqslant 0 .
$$

Полученное противоречие показывает, что $x_{\varepsilon}=0$ и, значит, минимум в $\varepsilon$-задаче равен нулю. Поэтому точка $(\varepsilon h, 1)$ также является ее решением, что доказывает требуемое.

Рассмотрим семейство решений $\left\{x_{\varepsilon}, \chi_{\varepsilon}\right\}$ в-задач. Из $\gamma(\chi) / \chi \rightarrow \infty$ при $\chi \rightarrow \infty$ вытекает ограниченность последовательности $\left\{\chi_{\varepsilon}\right\}$. Используя это и неравенство $f_{\varepsilon}\left(x_{\varepsilon}, \chi_{\varepsilon}\right) \leqslant 0$, имеем $\tilde{f}_{0}\left(x_{\varepsilon}\right) \leqslant \operatorname{const}\left|\tilde{f}_{0}(\varepsilon h)\right| \rightarrow 0, \varepsilon \rightarrow 0$. Поэтому $f_{0}(\tilde{x})+|\tilde{x}|^{4} \leqslant 0$ для любой предельной точки $\tilde{x}$ последовательности $\left\{x_{\varepsilon}\right\}$. В то же время $f_{0}(\tilde{x}) \geqslant 0$, так как $\tilde{x}$ удовлетворяет всем ограничениям задачи $(2.1)$. Значит, $\tilde{x}=0$ и, следовательно, доказано, что $x_{\varepsilon} \rightarrow 0$ при $\varepsilon \rightarrow 0$. Поэтому при всех малых $\varepsilon>0$, которые только и будут рассматриваться, $\left|x_{\varepsilon}\right|<\delta$.

Применим к решению $\left\{x_{\varepsilon}, \chi_{\varepsilon}\right\} \varepsilon$-задачи, для которого $\chi_{\varepsilon}>0$, необходимые условия, полученные на этапе I. Найдутся такие множитель Лагранжа $\lambda_{\varepsilon}=$ $\left(\lambda_{\varepsilon}^{0}, \ldots, \lambda_{\varepsilon}^{l}, y_{\varepsilon}^{*}\right)$ и линейное подпространство $\Pi_{\varepsilon}$, что $\left|\lambda_{\varepsilon}\right|=1, \lambda_{\varepsilon}^{j} \geqslant 0 \forall j$,

$$
\begin{gathered}
L\left(\varepsilon h, \lambda_{\varepsilon}\right)+\lambda_{\varepsilon}^{0} \varepsilon^{4}=\lambda_{\varepsilon}^{0} \gamma^{\prime}\left(\chi_{\varepsilon}\right) \geqslant 0, \quad \frac{\partial L}{\partial x}\left(x_{\varepsilon}, \lambda_{\varepsilon}\right)+4 \lambda_{\varepsilon}^{0} x_{\varepsilon}\left|x_{\varepsilon}\right|^{2}=0, \\
\Pi_{\varepsilon} \subseteq \operatorname{ker} F^{\prime}\left(x_{\varepsilon}\right), \quad \operatorname{codim} \Pi_{\varepsilon} \leqslant m, \\
\frac{\partial^{2} L}{\partial x^{2}}\left(x_{\varepsilon}, \lambda_{\varepsilon}\right)[x, x]+12 \lambda_{\varepsilon}^{0}\left|x_{\varepsilon}\right|^{2}|x|^{2} \geqslant 0 \quad \forall x \in \Pi_{\varepsilon} .
\end{gathered}
$$

При этом мы воспользовались тем, что ограничение $\chi \geqslant 0$ в функции Лагранжа для $\varepsilon$-задачи можно опустить, так как для рассматриваемого ее решения $\chi_{\varepsilon}>0$ и, значит, множитель Лагранжа, отвечающий этому ограничению, равен нулю (в силу условий дополняющей нежесткости).

Переходя к подпоследовательности, добьемся того, что $\left\{\lambda_{\varepsilon}\right\}$ сходится к некоторому единичному вектору $\lambda$. Переходя к пределу в полученных соотношениях, имеем $\lambda \in \Lambda\left(x_{0}\right)$. По теореме 2.3 существует такое подпространство П, что 
$\operatorname{codim} \Pi \leqslant m, \Pi \subseteq \operatorname{Ls}\left\{\Pi_{\varepsilon}\right\}$. Переходя к пределу при $\varepsilon \rightarrow 0$, как и на этапе I, получаем

$$
\Pi \subseteq \operatorname{ker} F^{\prime}\left(x_{0}\right), \quad \frac{\partial^{2} L}{\partial x^{2}}\left(x_{0}, \lambda\right)[x, x] \geqslant 0 \quad \forall x \in \Pi .
$$

Значит, $\lambda \in \Lambda_{m}\left(x_{0}\right)$. Наконец, раскладывая функцию $L\left(\cdot, \lambda_{\varepsilon}\right)$ до членов второго порядка и учитывая, что $h \in \mathscr{K}\left(x_{0}\right)$, из неравенства в $(2.8)$ получаем, что $\frac{\partial^{2} L}{\partial x^{2}}\left(x_{0}, \lambda\right)[h, h] \geqslant 0$. Последнее в силу произвольности $h$ завершает рассмотрения этапа II.

Этап III. Докажем теорему в полной общности. Возьмем произвольное $h \in$ $\mathscr{K}\left(x_{0}\right)$. Обозначим через $\widetilde{\mathscr{M}}$ множество таких подпространств $M \in \mathscr{M}$, что $h \in M, F^{\prime}\left(x_{0}\right)(M)=\operatorname{im} F^{\prime}\left(x_{0}\right)$. Возьмем произвольное $M \in \widetilde{\mathscr{M}}$ и рассмотрим задачу, полученную из (2.1) заменой $X$ на $M$. Как доказано на этапе II, для этой конечномерной задачи существуют такие множители Лагранжа $\lambda_{M}$, что

$$
\left|\lambda_{M}\right|=1, \quad \lambda_{M}^{j} \geqslant 0 \quad \forall j, \quad \frac{\partial L}{\partial x}\left(x_{0}, \lambda_{M}\right) \in M^{\perp}, \quad \frac{\partial^{2} L}{\partial x^{2}}\left(x_{0}, \lambda_{M}\right)[h, h] \geqslant 0,
$$

и существует подпространство $\Pi_{M} \subseteq \operatorname{ker} F^{\prime}\left(x_{0}\right)$, коразмерность которого относительно $M$ не превышает $m$ и для которого $\frac{\partial^{2} L}{\partial x^{2}}\left(x_{0}, \lambda_{M}\right)[x, x] \geqslant 0 \forall x \in \Pi_{M} \cdot^{3}$ Множество таких $\lambda_{M}$ обозначим через $\Lambda_{m}(M)$. В силу теоремы 2.3 оно, очевидно, замкнуто.

Для произвольных $M_{1}, \ldots, M_{s} \in \widetilde{\mathscr{M}}$, очевидно, $\bigcap_{i=1}^{s} \Lambda_{m}\left(M_{i}\right) \supseteq \Lambda_{m}\left(M_{1}+\cdots+\right.$ $\left.M_{s}\right) \neq \varnothing$. Следовательно, система непустых замкнутых множеств $\Lambda_{m}(M)$, $M \in \widetilde{\mathscr{M}}$, является центрированной. Поэтому в силу компактности единичной сферы в $\mathbb{R}^{m+1}$ пересечение $\bigcap \Lambda_{m}(M)$ непусто. Очевидно, для произвольного $M \in \widetilde{\mathscr{M}}$

вектора $\lambda$ из этого пересечения имеет место $\lambda \in \Lambda_{m}\left(x_{0}\right)$ и $\frac{\partial^{2} L}{\partial x^{2}}\left(x_{0}, \lambda\right)[h, h] \geqslant 0$. Теорема доказана.

Обсуждение теоремы 2.1. Если в задаче (2.1) ограничения типа неравенств отсутствуют (т. е. $l=0$ ), то в формулировке теоремы можно критический конус $\mathscr{K}\left(x_{0}\right)$ заменить подпространством ker $f^{\prime}\left(x_{0}\right)$ (так как в этом случае, если $h \in \operatorname{ker} f^{\prime}\left(x_{0}\right)$, то либо $h \in \mathscr{K}\left(x_{0}\right)$, либо $\left.(-h) \in \mathscr{K}\left(x_{0}\right)\right)$. Если при этом точка $x_{0}$ нормальна, то теорема 2.1 превращается в классические необходимые условия второго порядка (1.4), так как в нормальной точке множество $\Lambda\left(x_{0}\right)$ состоит из единственной точки и, значит, $\Lambda\left(x_{0}\right)=\Lambda_{k}\left(x_{0}\right)$ (при этом $(2.4)$ является следствием последнего равенства, так как в нормальной точке $\left.\Pi=\operatorname{ker} f^{\prime}\left(x_{0}\right)\right)$.

Наиболее сильными из известных ранее необходимых условий второго порядка для задачи (2.1) являются условия, полученные А.А. Милютиным (см. [7], [8]). Сформулируем их. Для этого обозначим через $\Lambda_{+}\left(x_{0}\right)$ множество тех $\lambda \in \Lambda\left(x_{0}\right)$, для которых индекс квадратичной формы $\frac{\partial^{2} L}{\partial x^{2}}\left(x_{0}, \lambda\right)$ конечен. Напомним, что индекс квадратичной формы $q$, обозначаемый через ind $q,-$ это

\footnotetext{
${ }^{3} M^{\perp}$ означает аннулятор подпространства $M$.
} 
максимум из размерностей всех тех подпространств, на которых она отрицательно определена или, что то же самое, - это минимальная из коразмерностей подпространств, на которых $q$ неотрицательно определена. Теорема Милютина заключается в том, что если $x_{0}$ - точка локального минимума, то выполняется условие, полученное из (2.4) заменой множества $\Lambda_{m}\left(x_{0}\right)$ на $\Lambda_{+}\left(x_{0}\right)$. Однако если пространство $X$ конечномерно, то, очевидно, $\Lambda_{+}\left(x_{0}\right)=\Lambda\left(x_{0}\right)$ и, значит, если точка $x_{0}$ анормальна для отображения $f$, т. е. $\operatorname{im} f^{\prime}\left(x_{0}\right) \neq Y$, то условия Милютина выполняются автоматически (так как в этом случае $\Lambda\left(x_{0}\right)$ содержит две точки: $\lambda_{1}=\left(0, \ldots, 0, y^{*}\right)$ и $\left.\lambda_{2}=-\lambda_{1}\right)$ и содержательной информации не несут. Но в любом случае необходимые условия Милютина [7], [8] слабее содержащихся в теореме 2.1, так как всегда $\Lambda_{m}\left(x_{0}\right) \subseteq \Lambda_{+}\left(x_{0}\right)$.

Впервые необходимые условия второго порядка, заключающиеся в том, что $\Lambda_{m}\left(x_{0}\right) \neq \varnothing$, без априорного предположения нормальности были получены для задачи оптимального быстродействия в [9], а для общей задачи оптимального управления и для задачи математического программирования в [10]. Условия (2.4) получены в [4].

Отметим важную особенность, которую вносят ограничения типа неравенств. Пусть в точке $x_{0}$ выполнено условие регулярности Мангасариана-Фромовица:

$$
\operatorname{im} f^{\prime}\left(x_{0}\right)=Y, \quad \exists d \in \operatorname{ker} f^{\prime}\left(x_{0}\right): \quad\left\langle f_{j}^{\prime}\left(x_{0}\right), d\right\rangle<0, \quad j=\overline{1, l},
$$

являющееся естественным обобщением условия Люстерника на задачи с неравенствами. Тогда $\lambda^{0}>0 \forall \lambda \in \Lambda\left(x_{0}\right)$, однако может не существовать "универсального" множителя Лагранжа $\lambda$, для которого $\frac{\partial^{2} L}{\partial x^{2}}\left(x_{0}, \lambda\right)[x, x] \geqslant 0 \forall x \in$ $\mathscr{K}\left(x_{0}\right)$. Соответствующий пример при $X=\mathbb{R}^{3}, l=4$ приведен в $[11 ; \S 2$, п. 2.4, c. 159].

Условие (2.4) теоремы можно представить в следующем эквивалентном виде. Для каждого $y \in \mathbb{R}^{l} \times \mathbb{R}^{k}$ положим

$$
\begin{gathered}
\omega(y)=\inf \left\{f_{0}^{\prime \prime}\left(x_{0}\right)[x, x], x \in \Omega(y)\right\} \\
\forall y: \Omega(y)=\left\{x \in \mathscr{K}\left(x_{0}\right): y=F^{\prime \prime}\left(x_{0}\right)[x, x]\right\} \neq \varnothing,
\end{gathered}
$$

причем этот инфимум может принимать и значение $-\infty$. Как легко видеть, (2.4) равносильно тому, что

$$
\forall y: \Omega(y) \neq \varnothing \quad \exists \lambda=\left(\lambda^{0}, \lambda^{1}, \ldots, \lambda^{l}, y^{*}\right) \in \Lambda_{m}\left(x_{0}\right): \quad \lambda^{0} \omega(y)+\langle\bar{\lambda}, y\rangle \geqslant 0,
$$

где $\bar{\lambda}=\left(\lambda^{1}, \ldots, \lambda^{l}, y^{*}\right)$. При этом, если $\omega(y)=-\infty$, то последнее неравенство в $(2.9)$ означает, что $\lambda^{0}=0$ и $\langle\bar{\lambda}, y\rangle \geqslant 0$.

Некоторые обобщения. При исследовании задач оптимального управления с геометрическими ограничениями (см. раздел 7 ниже) удобно использовать следующий вариант теоремы 2.1. Пусть $X$ - нормированное пространство, а $C \subseteq X-$ выпуклый замкнутый конус. Рассмотрим задачу

$$
f_{0}(x) \rightarrow \min , \quad f_{j}(x) \leqslant 0, \quad j=\overline{1, l}, \quad f(x)=0, \quad x \in C,
$$

которая отличается от (2.1) дополнительным ограничением $x \in C$. 
Приведем необходимые условия минимума для задачи $(2.10)$ в точке $x_{0}$. Обозначим через $\tilde{\Lambda}\left(x_{0}\right)$ множество нормированных множителей Лагранжа $\lambda$, отвечающих точке $x_{0}$ в силу принципа Лагранжа в задаче (2.10):

$$
\left\langle\frac{\partial L}{\partial x}\left(x_{0}, \lambda\right), x_{0}\right\rangle=0, \quad \frac{\partial L}{\partial x}\left(x_{0}, \lambda\right) \in C^{*}, \quad \lambda^{j} \geqslant 0 \quad \forall j, \quad \sum_{j=0}^{l} \lambda^{j}+\left|y^{*}\right|=1 .
$$

Здесь $C^{*}=\left\{\xi \in X^{*}:\langle\xi, x\rangle \geqslant 0 \forall x \in C\right\}-$ конус, сопряженный к конусу $C$. Положим

$$
\widetilde{\mathscr{K}}\left(x_{0}\right)=\left\{x \in C+\operatorname{span}\left\{x_{0}\right\}:\left\langle f_{j}^{\prime}\left(x_{0}\right), x\right\rangle \leqslant 0 \forall j, f^{\prime}\left(x_{0}\right) x=0\right\} .
$$

Положим $N=C \cap(-C)$ (и, значит, $N$ - максимальное линейное подпространство, лежащее в $C$ ). Обозначим через $\tilde{\Lambda}_{m}\left(x_{0}\right)$ множество тех множителей Лагранжа $\lambda \in \tilde{\Lambda}\left(x_{0}\right)$, для которых существует линейное подпространство $\Pi \subseteq N$, удовлетворяющее (2.3), где $s=m=k+l$ и codim означает коразмерность относительно подпространства $N$.

ТЕОРема 2.4. Пусть в задаче (2.10) точка $x_{0}$ является локальным минимумом. Тогда

$$
\Lambda=\tilde{\Lambda}_{m}\left(x_{0}\right) \neq \varnothing, \quad \max _{\lambda \in \Lambda} \frac{\partial^{2} L}{\partial x^{2}}\left(x_{0}, \lambda\right)[x, x] \geqslant 0 \quad \forall x \in \widetilde{\mathscr{K}}\left(x_{0}\right) .
$$

Доказательство этой теоремы аналогично доказательству теоремы 2.1 и приведено в [6]. В условии (2.11) важную роль играет структура конуса $\widetilde{\mathscr{K}}\left(x_{0}\right)$. Дело в том, что он может оказаться незамкнутым. Это является следствием того, что если конус $C$ не является конечногранным (т. е. не представим в виде пересечения конечного числа полупространств), а точка $x_{0}$ принадлежит границе $C$ и $x_{0} \neq 0$, то конус $C+\operatorname{span}\left\{x_{0}\right\}$, участвующий в определении $\widetilde{\mathscr{K}}\left(x_{0}\right)$, не обязан быть замкнутым. В то же время, в силу компактности множества $\tilde{\Lambda}_{m}\left(x_{0}\right)$ и непрерывности функции максимума по компактному множеству, неравенство (2.11) справедливо для всех $x \in \mathrm{cl} \widetilde{\mathscr{K}}\left(x_{0}\right)$. Здесь и ниже сl означает замыкание множества.

В связи с этим возникает естественный вопрос: останется ли справедливым условие $(2.11)$, если в нем конус $\widetilde{\mathscr{K}}\left(x_{0}\right)$ заменить на конус

$$
\left\{x \in \operatorname{cl}\left(C+\operatorname{span}\left\{x_{0}\right\}\right):\left\langle f_{j}^{\prime}\left(x_{0}\right), x\right\rangle \leqslant 0 \forall j, f^{\prime}\left(x_{0}\right) x=0\right\} ?
$$

(Заметим, что $\operatorname{cl}\left(C+\operatorname{span}\left\{x_{0}\right\}\right)$ совпадает с касательным конусом $T_{C}\left(x_{0}\right)$ к выпуклому конусу $C$ в точке $x_{0}$.) Пример задачи

$$
f_{0}(x)=x_{2}-x_{1}^{2} \rightarrow \min , \quad x \in C, \quad x_{3}-1=0, \quad x=\left(x_{1}, x_{2}, x_{3}\right) \in \mathbb{R}^{3},
$$

где $C$ - замкнутая коническая оболочка множества $\left\{x: x_{2} \geqslant x_{1}^{2}, x_{3}=1\right\}$ и $x_{0}=(0,0,1)$, дает на этот вопрос отрицательный ответ (подробнее см. [12]). 
В то же время в условиях второго порядка (2.11) можно вместо конуса $\widetilde{\mathscr{K}}\left(x_{0}\right)$ рассматривать конус (2.12), но для этого нужно в (2.11) к квадратичной форме $\frac{\partial^{2} L}{\partial x^{2}}\left(x_{0}, \lambda\right)[x, x]$ добавить некоторое слагаемое. Поясним сказанное на примере следующей более общей задачи (см. [13]):

$$
f_{0}(x) \rightarrow \min , \quad F(x) \in C,
$$

где $F: X \rightarrow Y=\mathbb{R}^{k}$. Здесь $C$ - произвольное замкнутое подмножество $Y$ (множество $C$ не обязательно выпуклое). Отметим, что в [13] рассмотрена даже более общая, чем (2.13), задача, а именно задача с дополнительным ограничением $x \in \widetilde{C}$, где $\widetilde{C}$ - замкнутое в конечной топологии (не обязательно выпуклое) подмножество $X$.

Для формулировки теоремы введем нужные понятия. Пусть $y_{0}=F\left(x_{0}\right) \in C$. Напомним, что вектор $d$ называется касательным к множеству $C$ в точке $y_{0}$, если существует такая последовательность $\left\{\varepsilon_{n}\right\} \downarrow 0$, что

$$
\operatorname{dist}\left(y_{0}+\varepsilon_{n} d, C\right)=o\left(\varepsilon_{n}\right) .
$$

Здесь $\operatorname{dist}(y, C)=\inf _{\xi \in C}\{\|\xi-y\|\}-$ расстояние от точки $y$ до множества $C$. Множество касательных векторов к множеству $C$ в точке $y_{0}$ образует конус (конус Булигана), который обозначается через $T_{C}\left(y_{0}\right)$.

Через $N\left(C ; y_{0}\right)$ обозначим нормальный конус Мордуховича к множеству $C$ в точке $y_{0}$ (см. [13]). Определим внешнее тангенциальное множество второго порядка к множеству $C$ в точке $y_{0} \in C$ по направлению $d \in T_{C}\left(y_{0}\right)$ по формуле

$$
O_{C}^{2}\left(y_{0}, d\right)=\left\{w \in Y: \exists\left\{\varepsilon_{n}\right\} \downarrow 0, \operatorname{dist}\left(y_{0}+\varepsilon_{n} d+\frac{1}{2} \varepsilon_{n}^{2} w, C\right)=o\left(\varepsilon_{n}^{2}\right)\right\} .
$$

Линейное подпространство $P \subseteq Y$ будем называть локально инвариантным подпространством относительно $C$ в точке $y_{0} \in C$ (ИП), если $\exists \delta>0:(C \cap$ $\left.B\left(y_{0}, \delta\right)\right)+(P \cap B(0, \delta)) \subseteq C$, где $B(y, \delta)$ - шар радиуса $\delta$ с центром в точке $y$. Очевидно, нулевое подпространство является ИП. В силу конечномерности $Y$ существует максимальное по включению ИП, которое обозначим через $\mathscr{I}_{C}$. Положим

$$
\begin{gathered}
\mathscr{K}^{C}\left(x_{0}\right)=\left\{x: F^{\prime}\left(x_{0}\right) x \in T_{C}\left(y_{0}\right),\left\langle f_{0}^{\prime}\left(x_{0}\right), x\right\rangle \leqslant 0\right\} \\
\lambda=\left(\lambda^{0}, y^{*}\right), \quad \lambda^{0} \in \mathbb{R}, \quad y^{*} \in Y^{*} \\
\Lambda\left(C ; x_{0}\right)=\left\{\lambda: \lambda^{0} \geqslant 0, y^{*} \in N\left(C ; y_{0}\right), \frac{\partial L}{\partial x}\left(x_{0}, \lambda\right)=0,|\lambda|=1\right\} .
\end{gathered}
$$

(Здесь функция Лагранжа $L$, естественно, определяется по формуле (1.2)).

Рассмотрим множество тех множителей Лагранжа $\lambda \in \Lambda\left(C ; x_{0}\right)$, для которых существует такое (зависящее от $\lambda$ ) подпространство $\Pi \subseteq X$, что

$$
\operatorname{codim} \Pi \leqslant k, \quad F^{\prime}\left(x_{0}\right)(\Pi) \subseteq \mathscr{I}_{C}, \quad \frac{\partial^{2} L}{\partial x^{2}}\left(x_{0}, \lambda\right)[x, x] \geqslant 0 \quad \forall x \in \Pi .
$$

Множество таких множителей Лагранжа обозначим через $\Lambda_{k}\left(C ; x_{0}\right)$. 
Теорема 2.5. Пусть в задаче (2.13) точка $x_{0}$ является локальным минимумом относительно конечной топологии. Тогда множество $\Lambda_{k}\left(C ; x_{0}\right)$ непусто и, более того, для любих $x \in \mathscr{K}^{C}\left(x_{0}\right) u w \in O_{C}^{2}\left(F\left(x_{0}\right), F^{\prime}\left(x_{0}\right) x\right)$ имеет место неравенство

$$
\max _{\lambda \in \Lambda}\left(\frac{\partial^{2} L}{\partial x^{2}}\left(x_{0}, \lambda\right)[x, x]-\left\langle y^{*}, w\right\rangle\right) \geqslant 0, \quad \text { əде } \quad \lambda=\left(\lambda^{0}, y^{*}\right), \quad \Lambda=\Lambda_{k}\left(C ; x_{0}\right) .
$$

Доказательство этой теоремы приведено в [13]. В силу теоремы о минимаксе (см. [13; приложение А]) из (2.15) вытекает справедливость следующего утверждения.

В предположениях теоремы 2.5 для любого выпуклого подмножества $\mathscr{T}(x) \subseteq$ $O_{C}^{2}\left(F\left(x_{0}\right), F^{\prime}\left(x_{0}\right) x\right)$ имеет место неравенство

$$
\max _{\lambda \in \Lambda}\left(\frac{\partial^{2} L}{\partial x^{2}}\left(x_{0}, \lambda\right)[x, x]-\sigma\left(y^{*}, \mathscr{T}(x)\right)\right) \geqslant 0, \quad \text { где } \quad \Lambda=\operatorname{conv} \Lambda_{k}\left(C ; x_{0}\right) .
$$

Здесь $\sigma(\cdot, T)$ - опорная функция множества $T \subseteq X$, т. е. $\sigma\left(y^{*}, T\right)=\sup _{y \in T}\left\langle y^{*}, y\right\rangle$ для $y^{*} \in Y$, a conv - выпуклая оболочка множества.

Обсудим теорему 2.5. Она естественным образом обобщает результаты [14], [15], где дополнительно предполагалось, что либо выполнено условие регулярности Робинсона $0 \in \operatorname{int}\left(F\left(x_{0}\right)+\operatorname{im} F^{\prime}\left(x_{0}\right)-C\right.$ ), либо $\operatorname{int} C \neq \varnothing$ (подробнее см. [13], [16]).

Пусть $F^{\prime}\left(x_{0}\right) x \in \mathscr{I}_{C}$. Тогда $0 \in O_{C}^{2}\left(F\left(x_{0}\right), F^{\prime}\left(x_{0}\right) x\right)$ и при $w=0$ из $(2.15)$ имеем

$$
\max _{\lambda \in \Lambda} \frac{\partial^{2} L}{\partial x^{2}}\left(x_{0}, \lambda\right)[x, x] \geqslant 0 .
$$

Если множество $C$ выпукло, то $\sigma\left(y^{*}, \mathscr{T}(x)\right) \leqslant 0$ для всех $y^{*}$ таких, что $\left(\lambda^{0}, y^{*}\right) \in$ $\Lambda\left(C ; x_{0}\right)$ для некоторого $\lambda^{0} \geqslant 0$ (см. [14; с. 178] или [13; с. 4]). Если же $C-$ выпуклый конечногранный конус (например, при $C=\mathbb{R}_{-}^{l} \times \mathbb{R}^{k}$, где $\mathbb{R}_{-}^{l}$ - неположительный ортант, мы приходим к задаче $(2.1))$, то $\sigma\left(y^{*}, \mathscr{T}(x)\right)=0$ для всех указанных $y^{*}$.

В то же время, если множество $C$ выпуклым не является, то член $\sigma\left(y^{*}, \mathscr{T}(h)\right)$ может оказаться строго положительным. Действительно, пусть $C=\{y=$ $\left.\left(y_{1}, y_{2}\right) \in \mathbb{R}^{2}: y_{1}^{l} \geqslant y_{2}^{m}\right\}$, где $l, m$ - натуральные числа, причем $m<l \leqslant 2 m$. Возьмем $h=(1,0) \in T_{C}(0)$. Тогда множество $O_{C}^{2}(0, h)$ содержит некоторый шар $B$ с центром в нуле и, значит, $\sigma(\zeta, B)>0 \forall \zeta \neq 0$. Пусть $l=2, m=1$, $f_{0}(x)=x_{1}^{2}-x_{2}, Y=\mathbb{R}^{2}, F(x) \equiv x$. Тогда $x_{0}=0$ является решением задачи $(2.13), h \in \mathscr{K}^{C}(0), y^{*}=-f_{0}^{\prime}(0)$, но $\sigma\left(-f_{0}^{\prime}(0), B\right)>0$.

Анормальные задачи. Вернемся к задаче (2.1). Если для отображения $F$ точка $x_{0}$ нормальна, то множество $\Lambda\left(x_{0}\right)$ состоит из единственной точки и, значит, теорема 2.1 дает классические необходимые условия второго порядка (при $l=0$ превращающиеся в (1.4)). Поэтому в нормальном случае их усилить нельзя. Если же для отображения $F$ точка $x_{0}$ анормальна, т. е. оператор $F^{\prime}\left(x_{0}\right)$ не является сюръективным, то теорему 2.1 усилить можно, заменив в ней множество $\Lambda_{m}\left(x_{0}\right)$ на меньшее. Сформулируем соответствующее утверждение. 
Пусть множество $C \subseteq Y$ выпукло и замкнуто. Определим внутреннее тангенциальное множество второго порядка к множеству $C$ в точке $y \in C$ по направлению $d \in T_{C}(y)$ :

$$
T_{C}^{2}(y, d)=\left\{w \in Y: \operatorname{dist}\left(y+\varepsilon d+\frac{1}{2} \varepsilon^{2} w, C\right)=o\left(\varepsilon^{2}\right), \varepsilon \geqslant 0\right\} .
$$

Оно выпукло, замкнуто и $T_{C}^{2}(y, d) \subseteq O_{C}^{2}(y, d)$, хотя в последнем включении равенства может и не быть (см. [14]).

Теорема 2.6. Пусть в задаче (2.13) точка $x_{0}$ является локальным минимумом относительно конечной топологии $u \operatorname{im} F^{\prime}\left(x_{0}\right)+\mathscr{I}_{C} \neq Y$ (в этом случае будем называть точку $x_{0}$ анормальной). Тогда множество $\Lambda_{k-1}\left(C ; x_{0}\right)$ непусто и для любого $x \in \mathscr{K}^{C}\left(x_{0}\right)$ имеет место неравенство

$$
\begin{gathered}
\max _{\lambda \in \Lambda}\left(\frac{\partial^{2} L}{\partial x^{2}}\left(x_{0}, \lambda\right)[x, x]-\sigma\left(y^{*}, T_{C}^{2}\left(F\left(x_{0}\right), F^{\prime}\left(x_{0}\right) x\right)\right)\right) \geqslant 0 \\
\text { əде } \Lambda=\operatorname{conv} \Lambda_{k-1}\left(C ; x_{0}\right) .
\end{gathered}
$$

Отметим, что в теореме 2.6 (так же как и в теореме 2.5) вместо максимального по включению ИП $\mathscr{I}_{C}$ можно брать любое ИП, например, нулевое подпространство.

Доказательство теоремы 2.6, использующее результаты вещественной алгебраической геометрии, приведено в [17], а при $C=\{0\}-$ в [18]. При $C=$ $\mathbb{R}_{-}^{l} \times\{0\} \subset \mathbb{R}^{l} \times \mathbb{R}^{k}$ получаем задачу (2.1) и, значит, из теоремы 2.6 вытекает следующая теорема.

Теорема 2.7. Пусть в задаче (2.1) точка $x_{0}$ является локальным минимумом относительно конечной топологии и точка $x_{0}$ анормальна для отображения $F\left(\right.$ m.e. $\left.\operatorname{im} F^{\prime}\left(x_{0}\right) \neq Y\right)$. Тогда множество $\Lambda_{m-1}\left(x_{0}\right)$ непусто и

$$
\max _{\lambda \in \Lambda} \frac{\partial^{2} L}{\partial x^{2}}\left(x_{0}, \lambda\right)[x, x] \geqslant 0 \quad \forall x \in \mathscr{K}\left(x_{0}\right), \quad \text { əде } \quad \Lambda=\Lambda_{m-1}\left(x_{0}\right) .
$$

Заметим, что пример задачи (1.5) показывает, что в теореме 2.6 даже при $k \geqslant 2$ заменить $(k-1)$ на $(k-2)$, вообще говоря, нельзя.

“Зазор” между необходимыми и достаточными условиями второго порядка. Важнейшей характеристикой любых необходимых условий второго порядка является величина "зазора" между ними и достаточными условиями второго порядка. Представляется естественным считать этот "зазор" минимально возможным (по отношению к необходимым условиям второго порядка), если необходимые условия превращаются в достаточные после сколь угодно малых в метрике $C^{2}$ возмущений минимизируемой функции и отображения, задающего ограничения, но не изменяющих их значений и значений их первых производных в исследуемой точке. Выясним, когда для задачи (2.1) указанный "зазор" является минимально возможным применительно к полученным выше необходимым условиям второго порядка. 
Пусть $X$ - гильбертово пространство. Предположим вначале, что для отображения $F$ точка $x_{0}$ нормальна и в ней выполнены необходимые условия второго порядка (2.4). Тогда в силу достаточных условий второго порядка (см. [7], [5] или теорему 4.1 из раздела 4 ниже) для произвольного $\varepsilon>0$ для функции $f_{0, \varepsilon}(x)=f_{0}(x)+\varepsilon\left|x-x_{0}\right|^{2}$ при ограничениях задачи (2.1) точка $x_{0}$ является строгим локальным минимумом (правда, размер окрестности, определяющей локальный минимум, может стремиться к нулю при $\varepsilon \rightarrow 0)$.

Предположим теперь, что для отображения $F$ точка $x_{0}$ анормальна. Оказывается, что тогда все зависит от того, содержит ли выпуклая оболочка conv $\Lambda_{m-1}\left(x_{0}\right)$ нуль или нет. Дело в том, что в (2.18) максимум по множеству $\Lambda=\Lambda_{m-1}\left(x_{0}\right)$ совпадает с максимумом по его выпуклой оболочке. Поэтому, если $0 \in \operatorname{conv} \Lambda$, то (2.18) выполняется автоматически. В этом случае условие (2.18) выполняется при любой минимизируемой функции $f_{0}$ и, значит, для задачи минимизации (2.1) содержательной информации не несет. Здесь повторяется та же ситуация, что и с правилом множителей Лагранжа в анормальной точке. Поэтому, если $0 \in \operatorname{conv} \Lambda$, то, вообще говоря, не следует ожидать, что при малых в $C^{2}$ возмущениях $f, f_{j}$, не изменяющих их значений и значений их первых производных в точке $x_{0}$, она станет локальным минимумом в возмущенной задаче. Сказанное подтверждает пример задачи

$$
\begin{gathered}
f_{0}(x)=x_{1} \rightarrow \min ; \\
x_{1} x_{2}=0, \quad x_{1} x_{3}=0, \quad l=0, \quad m=k=2, \quad x=\left(x_{1}, x_{2}, x_{3}\right) \in \mathbb{R}^{3} .
\end{gathered}
$$

В ней $0 \in \operatorname{conv} \Lambda_{1}(0)$, и ни при каких указанных выше возмущениях точка $x_{0}=0$ локальным минимумом не станет (это вытекает из доказываемой ниже теоремы 3.1). Совершенно по-иному обстоит дело, когда $0 \notin \operatorname{conv} \Lambda$.

Для целых $s \geqslant 0$ через $\mathscr{F}_{s}^{2}(x)$ обозначим множество таких $y^{*} \in Y^{*},\left|y^{*}\right|=1$, что $f^{\prime}(x)^{*} y^{*}=0$ и в $X$ существует подпространство П, для которого

$$
\begin{gathered}
\Pi \subseteq \operatorname{ker} f^{\prime}(x), \quad \operatorname{codim} \Pi \leqslant s ; \\
\left\langle\frac{\partial^{2} f}{\partial x^{2}}(x)[\xi, \xi], y^{*}\right\rangle \geqslant 0 \quad \forall \xi \in \Pi .
\end{gathered}
$$

Очевидно, если точка $x$ нормальна для отображения $f$, то $\mathscr{F}_{s}^{2}(x)=\varnothing \forall s$.

ОПРЕдЕлЕниЕ. Отображение $f$ называется 2-нормальным в точке $x$, если $0 \notin \operatorname{conv} \mathscr{F}_{k-1}^{2}(x)$ (в частности, если $\mathscr{F}_{k-1}^{2}(x)=\varnothing$ ). Отображение $f$ называется 2-нормальным, если оно 2-нормально в каждой точке.

Очевидно, $y^{*} \in \mathscr{F}_{k-1}^{2}(x) \Leftrightarrow\left(0, y^{*}\right) \in \Lambda_{k-1}(x)$ (здесь $l=0, m=k$ ). Данное определение является геометрическим и малопригодно для проверки 2-нормальности. В [5; гл. 1, § 1.9], приведены достаточные условия 2-нормальности, сформулированные в терминах отображения $f$. Там же доказано, что если $X=\mathbb{R}^{n}$ и $n \gg k$ (например, $\left.n>2(k-2),(n-k-1)(n-k)>2(k-1)\right)$, то условие 2-нормальности является условием общего положения, а именно, в пространстве $C_{s}^{3}\left(\mathbb{R}^{n}, \mathbb{R}^{k}\right)$, наделенном топологией Уитни (см. [19; гл. 2, §1]), множество 2-нормальных отображений массивно (т. е. содержит пересечение счетного числа всюду плотных открытых множеств) и, следовательно, само всюду плотно. 
Теорема 2.8. Пусть $X$ - гильбертово пространство. Предположим, что отображение $f$ является 2-нормальным в точке $x_{0}$ и в ней выполняются необходимые условия второго порядка (2.18). Тогда существует такой вектор $\bar{y} \in Y$, что для любого $\varepsilon>0$ в возмущенной задаче

$$
\begin{gathered}
f_{0, \varepsilon}(x)=f_{0}(x)+\varepsilon\left|x-x_{0}\right|^{2} \rightarrow \min , \\
f_{j, \varepsilon}(x)=f_{j}(x)+\varepsilon\left|x-x_{0}\right|^{2} \leqslant 0, \quad j=\overline{1, l}, \quad f_{\varepsilon}(x)=f(x)+\varepsilon\left|x-x_{0}\right|^{2} \bar{y}=0
\end{gathered}
$$

точка $x_{0}$ доставляет строгий локальный минимум.

Доказательство этой теоремы основано на достаточных условиях второго порядка и приведено в [5; гл. $1, \S 1.8]$.

Доказательство теоремы 2.3. Для произвольного банахова пространства оно приведено в [5], а здесь мы ограничимся доказательством для частного случая, предполагая, что $X$ - гильбертово пространство, что существенно облегчает рассмотрения. Впервые теорема 2.3 была приведена в [9] именно для гильбертова случая.

Не теряя общности, будем считать, что $\operatorname{codim} \Pi_{i}=k \forall i$. В каждом ортогональном дополнении $\Pi_{i}^{\perp}$ выберем свой ортонормированный базис $e_{i, 1}, \ldots, e_{i, k}$. Переходя к подпоследовательностям, будем считать, что для каждого натурального $j \in\{1, \ldots, k\}$ последовательность $\left\{e_{i, j}\right\}$ при $i \rightarrow \infty$ слабо сходится к вектору $e_{j}$. Покажем, что подпространство $\Pi=\left\{x \in X:\left\langle e_{j}, x\right\rangle=0 \forall j \in\right.$ $\{1, \ldots, k\}\}$ является искомым. Для этого достаточно, взяв произвольную точку $x_{0} \in \Pi$, построить сходящуюся к ней последовательность $\left\{x_{i}\right\}: x_{i} \in \Pi_{i} \forall i$. Сделаем это. Для этого при каждом $i$ рассмотрим задачу минимизации

$$
\left|x-x_{0}\right|^{2} \rightarrow \min , \quad\left\langle e_{i, j}, x\right\rangle=0, \quad j=1, \ldots, k
$$

Как несложно видеть, каждая из этих задач имеет решение, которое обозначим через $x_{i}$. В силу принципа Лагранжа

$$
\exists \lambda_{i, j}: x_{i}-x_{0}=\sum_{j=1}^{k} \lambda_{i, j} e_{i, j} \Rightarrow\left|x_{i}-x_{0}\right|^{2}=\sum_{j=1}^{k} \lambda_{i, j}^{2} \Rightarrow\left|\lambda_{i, j}\right| \leqslant\left|x_{i}-x_{0}\right| \quad \forall i, j .
$$

Поэтому, умножая первое равенство скалярно на $x_{i}-x_{0}$, имеем

$$
\begin{aligned}
& \left|x_{i}-x_{0}\right|^{2}=-\sum_{j=1}^{k} \lambda_{i, j}\left\langle e_{i, j}, x_{0}\right\rangle \leqslant \sum_{j=1}^{k}\left|\lambda_{i, j}\right|\left|\left\langle e_{i, j}, x_{0}\right\rangle\right| \leqslant\left|x_{i}-x_{0}\right| \sum_{j=1}^{k}\left|\left\langle e_{i, j}, x_{0}\right\rangle\right| \\
& \Rightarrow\left|x_{i}-x_{0}\right| \leqslant \sum_{j=1}^{k}\left|\left\langle e_{i, j}, x_{0}\right\rangle\right| \rightarrow \sum_{j=1}^{k}\left|\left\langle e_{j}, x_{0}\right\rangle\right|=0 .
\end{aligned}
$$

Теорема доказана. 


\section{3. Необходимые условия экстремума первого и второго порядка. 2-регулярность}

Обобщенная функция Лагранжа $\mathscr{L}_{\mathscr{A}}$. В этом разделе рассмотрим другой подход к исследованию необходимых условий в экстремальных задачах, основанный на обобщенной функции Лагранжа $\mathscr{L}_{\mathscr{A}}$ и понятии 2-регулярности. Чтобы прояснить суть дела, ограничимся рассмотрением задачи лишь с ограничениями типа равенств

$$
f_{0}(x) \rightarrow \min , \quad F(x)=0 .
$$

Здесь $f_{0}$ - заданная минимизируемая функция, $F: X \rightarrow Y$ - заданное отображение, а пространства $X$ и $Y$ те же, что и в предыдущем разделе. Пусть $x_{0}-$ точка локального (относительно конечной топологии) минимума в рассматриваемой задаче. В дальнейшем будем предполагать, что в окрестности точки $x_{0}$ функция $f_{0}$ непрерывно дифференцируема, а отображение $F$ трижды непрерывно дифференцируемо относительно конечной топологии (см. раздел 2), причем его третья производная удовлетворяет условию Липшица.

Введем необходимые понятия. Обозначим через $\pi$ оператор ортогонального проектирования $Y$ на $\left(\operatorname{im} F^{\prime}\left(x_{0}\right)\right)^{\perp}$. Рассмотрим непустой $(0 \in H)$ конус

$$
H=H\left(x_{0}\right)=\left\{h \in X: F^{\prime}\left(x_{0}\right) h=0, F^{\prime \prime}\left(x_{0}\right)[h, h] \in \operatorname{im} F^{\prime}\left(x_{0}\right)\right\} .
$$

Этот конус чрезвычайно важен для исследования допустимого множества $M=$ $\{x \in X: F(x)=0\}$. Действительно, как несложно видеть, если вектор $h$ является касательным к множеству $M$ в точке $x_{0}$, то $h \in H\left(x_{0}\right)$. Однако существенно важнее, что при дополнительных, но естественных предположениях справедливо обратное. Чтобы пояснить сказанное, введем важное понятие.

Для произвольного фиксированного $h \in H\left(x_{0}\right)$ определим линейный оператор $G\left(x_{0}, h\right): X \times \operatorname{ker} F^{\prime}\left(x_{0}\right) \rightarrow Y$ формулой

$$
G\left(x_{0}, h\right)\left(x_{1}, x_{2}\right)=F^{\prime}\left(x_{0}\right) x_{1}+F^{\prime \prime}\left(x_{0}\right)\left[h, x_{2}\right], \quad x_{1} \in X, \quad x_{2} \in \operatorname{ker} F^{\prime}\left(x_{0}\right) .
$$

ОПРЕДЕЛЕНИЕ. Отображение $F$ называется 2-регулярным в точке $x_{0}$ по направлению $h \in H\left(x_{0}\right)$, если оператор $G\left(x_{0}, h\right)$ сюръективен. ${ }^{4}$ Отображение $F$ называется 2-регулярным в точке $x_{0}$, если оно 2-регулярно в ней по любому ненулевому направлению $h \in H\left(x_{0}\right)$.

Понятие 2-регулярного направления было введено в другой (но эквивалентной) форме в [20]. Отметим, что если отображение нормально в некоторой точке, то оно в ней, очевидно, 2-регулярно, но, вообе говоря, не наоборот.

Итак, как было доказано в [20], если отображение $F$ является 2-регулярным в точке $x_{0}$ по направлению $h \in H\left(x_{0}\right)$, то вектор $h$ является касательным к множеству $M$ в точке $x_{0}$. (Это утверждение вытекает также из доказываемой ниже теоремы 3.3.) Таким образом, в частности, если отображение $F$ является 2 -регулярным в точке $x_{0}$, то конус $H\left(x_{0}\right)$ совпадает с касательным конусом

\footnotetext{
4Термин "направление" введен для образности, и мы считаем его синонимом понятия "вектор".
} 
к множеству $M$ в точке $x_{0}$. А описание касательного конуса к допустимому множеству в точке минимума дает возможность вывода необходимых условий минимума в экстремальной задаче.

Рассмотрим введенную в [20] функцию $\mathscr{L}_{\mathscr{A}}: X \times \mathbb{R} \times Y^{*} \times Y^{*} \times H\left(x_{0}\right) \rightarrow \mathbb{R}$ :

$$
\begin{gathered}
\mathscr{L}_{\mathscr{A}}\left(x, \lambda_{A}, h\right)=\lambda^{0} f_{0}(x)+\left\langle y_{1}^{*}, F(x)\right\rangle+\left\langle y_{2}^{*}, F^{\prime}(x) h\right\rangle, \\
\lambda_{A}=\left(\lambda^{0}, y_{1}^{*}, y_{2}^{*}\right), \quad \lambda^{0} \in \mathbb{R}, \quad y_{1}^{*}, y_{2}^{*} \in Y^{*}, \quad h \in H\left(x_{0}\right) .
\end{gathered}
$$

Вектор $\lambda_{A}$ и его компоненты также называются множителями Лагранжа. Отметим, что набор множителей Лагранжа $\lambda_{A}$ по сравнению с рассматривавшимся ранее классическим набором множителей Лагранжа $\lambda$ имеет дополнительную компоненту $y_{2}^{*}$, а функция $\mathscr{L}_{\mathscr{A}}$ отличается от функции Лагранжа $L$ наличием последнего слагаемого, содержащего $y_{2}^{*}$. Вектор $h$ в функции $\mathscr{L}_{\mathscr{A}}$ играет роль параметра.

ТеОрема 3.1. Пусть $x_{0}$ - точка локального относительно конечной топологии минимума в задаче (3.1). Тогда для любого $h \in H\left(x_{0}\right)$ существует $\lambda_{A}$ maкое, что

$$
\begin{gathered}
\frac{\partial \mathscr{L}_{\mathscr{A}}}{\partial x}\left(x_{0}, \lambda_{A}, h\right)=0, \\
y_{1}^{*} \in \operatorname{im} F^{\prime}\left(x_{0}\right) ; \quad y_{2}^{*} \in\left(\operatorname{im} F^{\prime}\left(x_{0}\right)\right)^{\perp}, \quad \lambda^{0} \geqslant 0, \quad \lambda^{0}+\left|y_{2}^{*}\right| \neq 0 .
\end{gathered}
$$

ТЕОрема 3.2. Пусть $x_{0}$ - точка локального относительно конечной топологии минимума в задаче (3.1) и в окрестности точки $x_{0}$ функиия $f_{0}$ дважды непрерывно дифференцируема относительно конечной топологии.

Тогда для любого вектора $h \in H\left(x_{0}\right)$ существует множитель Лагранжа $\lambda_{A}$, для которого выполняются соотношения (3.3), (3.4) и

$$
\frac{\partial^{2} \mathscr{L}_{\mathscr{A}}}{\partial x^{2}}\left(x_{0}, \lambda^{0}, y_{1}^{*}, \frac{1}{3} y_{2}^{*}, h\right)[h, h] \geqslant 0 .
$$

Теоремы 3.1 и 3.2 дают необходимые условия первого и второго порядков. Они были получены в [20] для случая, когда $X$ и $Y$ - банаховы пространства, отображения $f_{0}, F$ достаточное число раз дифференцируемы и подпространство im $F^{\prime}\left(x_{0}\right)$ замкнуто.

2-регулярность. Перед тем как доказывать эти теоремы, обсудим их. Если точка $x_{0}$ нормальна, то в силу $(3.4) y_{2}^{*}=0$, и функция $\mathscr{L}_{\mathscr{A}}$ превращается в обычную функцию Лагранжа $L$, множитель Лагранжа $\lambda_{A}=\left(\lambda^{0}, y_{1}^{*}, 0\right)$, после отбрасывания последней нулевой компоненты, превращается в обычный множитель Лагранжа $\lambda=\left(\lambda^{0}, y^{*}\right)$, а сама теорема 3.1 - в принцип Лагранжа (1.3). (Чтобы увидеть последнее, надо в теореме 3.1 взять $h=0$.) Теорема 3.2 при этом превращается в (1.4). Таким образом, в предположении нормальности приведенные теоремы эквивалентны классическим необходимым условиям второго порядка.

Если точка $x_{0}$ анормальна и, более того, отображение $F$ не является 2-регулярным по направлению $h \in H\left(x_{0}\right)$, то теореме 3.1 удовлетворяет множитель Лагранжа $\lambda_{A}=\left(0, y_{1}^{*}, y_{2}^{*}\right)$, где $y_{1}^{*}-$ произвольный ненулевой вектор из 
$\left(\operatorname{im} G\left(x_{0}, h\right)\right)^{\perp}$ и $y_{2}^{*}=\pi y_{1}^{*}$. Значит, в этом случае теорема 3.1 содержательной информации не несет (ведь $\lambda^{0}=0$ и минимизируемый функционал никак не участвует в условиях (3.3), (3.4)) и она является лишь расшифровкой нарушения условия 2-регулярности по направлению $h$. Теорема 3.2 в этом случае также содержательной информации не несет, так как наряду с множителем Лагранжа $\lambda_{A}$ условиям (3.3), (3.4) удовлетворяет также и $\left(-\lambda_{A}\right)$ и, значит, условие (3.5) выполняется автоматически.

Наиболее интересен третий случай, когда точка $x_{0}$ анормальна, но $F$ является 2-регулярным по направлению $h$. Тогда, как легко видеть, $\lambda^{0}>0$ и теорема 3.1 дает содержательную информацию относительно градиента минимизируемой функции, а теорема 3.2 - относительно ее второй производной. При этом из сюръективности линейного оператора $G\left(x_{0}, h\right)$ вытекает, что множители Лагранжа $\lambda_{A}$ определяются из условий $(3.3),(3.4)$ с точностью до нормировки единственным образом.

Доказательство теорем 3.1, 3.2. Оно основано на следующем утверждении.

Теорема 3.3. Пусть $h \in H\left(x_{0}\right)$ и $F$ является 2-регулярным в точке $x_{0}$ по направлению $h$. Тогда существуют $h_{2} \in X$ и конечномерное подпространство $\widetilde{X} \subseteq X$ maкuе, ито

$$
\begin{aligned}
& F^{\prime}\left(x_{0}\right) h_{2}+\frac{1}{2} F^{\prime \prime}\left(x_{0}\right)[h, h]=0, \quad F^{\prime \prime}\left(x_{0}\right)\left[h, h_{2}\right]+\frac{1}{6} F^{\prime \prime \prime}\left(x_{0}\right)[h, h, h] \in \operatorname{im} F^{\prime}\left(x_{0}\right), \\
& F(h(\varepsilon))=0 \quad \forall \varepsilon, \quad h(\varepsilon) \in \widetilde{X} \quad \forall \varepsilon, \quad \text { əде } \quad h(\varepsilon)=x_{0}+\varepsilon h+\varepsilon^{2} h_{2}+O\left(\varepsilon^{3}\right) .
\end{aligned}
$$

ДокАзАтельство. Для удобства здесь и ниже будем считать, что $x_{0}=0$, а в обозначении производных в точке $x_{0}$ зависимость от нее будем опускать (т. е. $f_{0}^{\prime}=f_{0}^{\prime}\left(x_{0}\right), F^{\prime \prime}=F^{\prime \prime}\left(x_{0}\right)$ и т. д.). В силу конечномерности $Y$, в $X$ существует такое конечномерное подпространство $\widetilde{X}$, что сужение линейного оператора $G\left(x_{0}, h\right)$ на $\widetilde{X} \times\left(\operatorname{ker} F^{\prime} \cap \tilde{X}\right)$ по-прежнему сюръективно. Поэтому, заменяя $X$ на $\widetilde{X}$, будем считать само $X$ конечномерным.

В силу условия 2-регулярности по направлению $h$ существуют $h_{2}, h_{3} \in X$ такие, что

$$
F^{\prime} h_{2}+\frac{1}{2} F^{\prime \prime}[h, h]=0, \quad F^{\prime} h_{3}+F^{\prime \prime}\left[h, h_{2}\right]+\frac{1}{6} F^{\prime \prime \prime}[h, h, h]=0
$$

(подробнее см. [5; $\S 5$, с. 72,73$])$. Будем искать $h(\varepsilon)$ при фиксированных $\varepsilon$ в виде

$$
h(\varepsilon)=\varepsilon h+\varepsilon^{2} h_{2}+\varepsilon^{3} h_{3}+\varepsilon^{2} r_{2}+\varepsilon^{3} r_{1},
$$

где неизвестные $r_{1}=r_{1}(\varepsilon) \in X$ и $r_{2}=r_{2}(\varepsilon) \in \operatorname{ker} F^{\prime}$ подлежат нахождению.

В окрестности нуля отображение $F$ представимо в виде

$$
F(x)=F^{\prime} x+\frac{1}{2} F^{\prime \prime}[x, x]+\frac{1}{6} F^{\prime \prime \prime}[x, x, x]+R(x)[x, x, x],
$$


где $R(x)$ при каждом $x$ - полилинейное отображение, $R(0)=0$ и $R(\cdot)$ локально липшицево. При фиксированных $\varepsilon \neq 0$ рассмотрим уравнение $F(h(\varepsilon))=0$ относительно неизвестных $r_{1} \in X, r_{2} \in \operatorname{ker} F^{\prime}$. В силу выписанного представления и (3.8), после деления на $\varepsilon^{3}$ это уравнение принимает вид

$$
G\left(x_{0}, h\right)\left(r_{1}, r_{2}\right)=\Delta\left(\varepsilon, r_{1}, r_{2}\right),
$$

где $\Delta$ - непрерывное отображение, для которого $\Delta\left(0, r_{1}, r_{2}\right) \equiv 0$ и отображение $\Delta(\varepsilon, \cdot, \cdot)$ на единичном шаре удовлетворяет условию Липшица с константой Липшица $k(\varepsilon)$ такой, что $k(\varepsilon) \rightarrow 0$ при $\varepsilon \rightarrow 0$. В силу сюръективности (накрываемости) оператора $G\left(x_{0}, h\right)$ к уравнению (3.9) применима теорема о существовании точек совпадения (теорема 1 из [21]). Из нее получаем, что для любого достаточно малого по модулю $\varepsilon$ существует такое решение $r_{1}(\varepsilon), r_{2}(\varepsilon)$ уравнения (3.9), что $\left|r_{1}(\varepsilon)\right|+\left|r_{2}(\varepsilon)\right| \leqslant$ const $|\varepsilon|$. Теорема доказана.

ДокАЗАТЕЛЬСтво теоремы 3.1. В силу сказанного выше нам достаточно рассмотреть случай, когда $F$ является 2-регулярным в точке $x_{0}=0$ по направлению $h$. Сделаем это. По теореме 3.3 векторы $h$ и $(-h)$ являются касательными к множеству $M=\{x: F(x)=0\}$ в точке $x_{0}=0$. Следовательно, $\left\langle f_{0}^{\prime}, h\right\rangle=0$.

Обозначим через $T_{H}(h)$ касательный конус к множеству $H=H\left(x_{0}\right)$ в точке $h$. Определим линейный оператор $\widetilde{G}(h): X \rightarrow Y$ формулой $\widetilde{G}(h) x=F^{\prime} x+$ $\pi F^{\prime \prime}[h, x]$. Из сюръективности оператора $G\left(x_{0}, h\right)$ вытекает сюръективность оператора $\widetilde{G}(h)$. Определим отображение $g: X \rightarrow Y$ по формуле $g(x)=\widetilde{G}(x) x$. Очевидно, что $H=\{x: g(x)=0\}$ и для отображения $g$ точка $h$ является нормальной. Поэтому по теореме Люстерника (о касательном конусе, см. [1]) $\operatorname{ker} \widetilde{G}(h) \subseteq T_{H}(h)$. Следовательно, для произвольного $x \in \operatorname{ker} \widetilde{G}(h)$ существуют такие сходящаяся к нулю последовательность положительных чисел $\left\{\alpha_{i}\right\}$ и сходящаяся к нулю последовательность векторов $\left\{a_{i}\right\}$ из $X$, что $h_{i}=h+$ $\alpha_{i} x+\alpha_{i} a_{i} \in H$ при всех больших $i$. Но в силу теоремы 3.3 все векторы, которые принадлежат $H$ и достаточно близки к $h$, также являются касательными к множеству $M$. Сказанное относится к векторам $h_{i}$ при больших $i$, и, следовательно, $\left\langle f_{0}^{\prime}, h_{i}\right\rangle \leqslant 0 \Rightarrow\left\langle f_{0}^{\prime}, x\right\rangle \leqslant 0 \forall x \in \operatorname{ker} \widetilde{G}(h)$. Следовательно, $\left\langle f_{0}^{\prime}, x\right\rangle=0$ $\forall x \in \operatorname{ker} \widetilde{G}(h)$, так как если $x$ принадлежит $\operatorname{ker} \widetilde{G}(h)$, то и $(-x)$ также принадлежит $\operatorname{ker} \widetilde{G}(h)$.

Итак, доказано, что

$$
\left\langle f_{0}^{\prime}, x\right\rangle=0 \quad \forall x \in X: \quad F^{\prime} x=0, \quad \pi F^{\prime \prime}[h, x]=0 .
$$

Отсюда в силу леммы об аннуляторе (см. [1; с. 26]) вытекает существование таких $y_{1}^{*}, y_{2}^{*}$, что для $\lambda_{A}=\left(1, y_{1}^{*}, y_{2}^{*}\right)$ выполняются условия (3.3) и (3.4). Теорема доказана.

ЗАмЕчАниЕ. Из доказательства теоремы 3.1, приведенного в [20], вытекает, что если $X$ - банахово пространство, то в теореме 3.1 достаточно предполагать лишь, что отображение $F$ дважды дифференцируемо по Фреше в точке $x_{0}$. Отметим, что в $[5 ; \S 1.13]$ получены необходимые условия экстремума высших порядков. 
ДоКАЗАТЕЛЬСтво теоремы 3.2. Возьмем $h_{2}$, для которого имеют место (3.6) и (3.7). Тогда в силу (3.7)

$$
\left\langle f_{0}^{\prime}, h_{2}\right\rangle+\frac{1}{2} f_{0}^{\prime \prime}[h, h] \geqslant 0 .
$$

Возьмем множитель Лагранжа $\lambda_{A}=\left(1, y_{1}^{*}, y_{2}^{*}\right)$, удовлетворяющий соотношениям (3.3) и (3.4). Из них в силу (3.6) имеем $\left\langle f_{0}^{\prime}, h_{2}\right\rangle=\left(\left\langle y_{1}^{*}, F^{\prime \prime}[h, h]\right\rangle+\right.$ $\left.\left\langle y_{2}^{*}, F^{\prime \prime \prime}[h, h, h]\right\rangle / 3\right) / 2$. Подставляя это выражение в (3.10), получаем (3.5). Теорема доказана.

Классический принцип Лагранжа в анормальных задачах. Следующее утверждение дает усиление теоремы 3.1 .

ТеОрема 3.4. Пусть $x_{0}$ - точка локалъного относительно конечной топологии минимума в задаче (3.1). Тогда, если отображение F является 2-регулярным по направлению $h \in H\left(x_{0}\right)$, то для него существует такой множитель Лагранжа $\lambda_{A}$, что выполняются условия (3.3), (3.4), а также

$$
\left\langle y_{2}^{*}, F^{\prime \prime}\left(x_{0}\right)[x, x]\right\rangle=0 \quad \forall x: F^{\prime}\left(x_{0}\right) x=0, F^{\prime \prime}\left(x_{0}\right)[h, x] \in \operatorname{im} F^{\prime}\left(x_{0}\right) .
$$

ДокАЗАТЕЛЬство. В силу теоремы 3.1 вектору $h$ соответствует единственный множитель Лагранжа с единичной первой координатой $\lambda_{A}=\left(1, y_{1}^{*}, y_{2}^{*}\right)$. Как было показано при доказательстве теоремы 3.1 , для произвольного $x \in$ $\operatorname{ker} \widetilde{G}(h)$ существуют сходящаяся к нулю последовательность положительных чисел $\left\{\alpha_{i}\right\}$ и последовательность векторов $\left\{a_{i}\right\} \subset X$, лежащая в некотором конечномерном подпространстве и сходящаяся к нулю, для которых $h_{i}=h+$ $\alpha_{i} x+\alpha_{i} a_{i} \in H\left(x_{0}\right) \forall i$ и отображение $F$ является 2-регулярным по направлениям $h_{i}$. Поэтому по теореме 3.1 для любых $i$ существуют множители Лагранжа $\lambda_{A}^{i}=\left(1, y_{1, i}^{*}, y_{2, i}^{*}\right)$, для которых имеет место (3.4) и

$$
\frac{\partial \mathscr{L}_{\mathscr{A}}}{\partial x}\left(x_{0}, \lambda_{A}^{i}, h_{i}\right)=0 .
$$

Из сюръективности оператора $\widetilde{G}(h)$ следует, что последовательности $\left\{y_{1, i}^{*}\right\}$ и $\left\{y_{2, i}^{*}\right\}$ ограничены. Поэтому, переходя к подпоследовательности, будем считать, что $\left\{y_{s, i}^{*}\right\} \rightarrow \tilde{y}_{s}^{*}, i \rightarrow \infty, s=1,2$. Очевидно, что $\tilde{\lambda}_{A}=\left(1, \tilde{y}_{1}^{*}, \tilde{y}_{2}^{*}\right)$ соответствует вектору $h$ в силу теоремы 3.1. Следовательно, $\tilde{\lambda}_{A}=\lambda_{A} \Rightarrow \lambda_{A}^{i} \rightarrow \lambda_{A}$. Поэтому, применяя левую часть $(3.12)$ к вектору $x \in \operatorname{ker} \widetilde{G}(h)$, учитывая, что $f_{0}^{\prime} \in(\operatorname{ker} \widetilde{G}(h))^{\perp}, y_{2, i}^{*} \in\left(\operatorname{im} F^{\prime}\left(x_{0}\right)\right)^{\perp}$, и осуществляя предельный переход при $i \rightarrow \infty$, получаем (3.11). Теорема доказана.

Следующий простой пример показывает, что в теореме 3.4 предположение о 2 -регулярности отображения $F$ по направлению $h$ существенно.

ПримеР 3.1. При натуральных $1 \leqslant m<n$ и $a \in \mathbb{R}^{n}, a \neq 0$ рассмотрим задачу

$$
f_{0}=\langle a, x\rangle \rightarrow \min , \quad F(x)=\sum_{i=1}^{m} x_{i}^{2}+\sum_{i=m+1}^{n} x_{i}^{4}=0 .
$$


Точка $x_{0}=0$ является ее решением, $H(0)=\left\{h: h_{i}=0, i=\overline{1, m}\right\} \Rightarrow$ $F^{\prime \prime}(0) h=0 \forall h \in H(0)$. Возьмем произвольное $h \in H(0), h \neq 0$. Для любых множителей Лагранжа $\lambda_{A}$, отвечающих выбранному $h$ в силу (3.3), (3.4), выполняется $\lambda^{0}=0 \Rightarrow y_{2}^{*} \neq 0$. В то же время $F^{\prime \prime}(0) \neq 0$ и, значит, при $y_{2}^{*} \neq 0$ условие (3.11) нарушается. Здесь все дело в том, что отображение $F$ не является 2-регулярным ни по одному направлению $h \in H(0)$.

Пусть в задаче (3.1) минимум достигается в анормальной точке $x_{0}$. Тогда в силу принципа Лагранжа (1.3) ей отвечает множитель Лагранжа $\bar{\lambda}=\left(0, \bar{y}^{*}\right)$. В то же время теорема 3.4 дает достаточные условия существования в этой задаче наряду с указанным множителем Лагранжа $\bar{\lambda}$ и так называемого нормального множителя Лагранжа $\lambda=\left(1, y^{*}\right)$. Последнее означает, что справедлив классический принцип Лагранжа

$$
\exists y^{*}: \quad f_{0}^{\prime}\left(x_{0}\right)+F^{\prime}\left(x_{0}\right)^{*} y^{*}=0,
$$

т. е. именно в том виде, в котором его сформулировал сам Лагранж.

Лемма 3.1. Пусть в задаче (3.1) $x_{0}$ является точкой локалъного относительно конечной топологии минимума и множество направлений $h \in H\left(x_{0}\right)$, по которым отображение $F$ в точке $x_{0}$ 2-регулярно, непусто. Предположим, что выполняется хотя бы одно из следующих предположений:

а) для любого ненулевого $y_{2}^{*} \in\left(\operatorname{im} F^{\prime}\left(x_{0}\right)\right)^{\perp}$ конус нулей квадратичной формы $\left\langle y_{2}^{*}, F^{\prime \prime}\left(x_{0}\right)[x, x]\right\rangle$, рассматриваемой на подпространстве $\operatorname{ker} F^{\prime}\left(x_{0}\right)$, не содержит никакого подпространства, коразмерность которого равна $\operatorname{codim}\left(\operatorname{im} F^{\prime}\left(x_{0}\right)\right)$;

b) отображение $F$ является 2-нормальным в точке $x_{0}$.

Тогда в точке $x_{0}$ выполняется классический приниип Лагранжа (3.13).

ДоказАтельство. Пусть выполняется а). Возьмем вектор $h \in H\left(x_{0}\right)$, вдоль которого отображение $F$ является 2-регулярным, и пусть множитель Лагранжа $\lambda_{A}=\left(\lambda^{0}, y_{1}^{*}, y_{2}^{*}\right)$ отвечает ему в силу теоремы 3.4. Докажем, что $y_{2}^{*}=0$.

Действительно, в силу (3.11) квадратичная форма $\left\langle y_{2}^{*}, F^{\prime \prime}\left(x_{0}\right)[x, x]\right\rangle$ обращается в нуль на подпространстве $\operatorname{ker} \widetilde{G}(h)$, коразмерность которого относительно содержащего его подпространства $\operatorname{ker} F^{\prime}\left(x_{0}\right)$ равна $\operatorname{codim}\left(\operatorname{im} F^{\prime}\left(x_{0}\right)\right)$. Отсюда в силу а) следует, что $y_{2}^{*}=0$, откуда в силу $(3.4) \lambda^{0}>0$. Поэтому из $(3.3)$ вытекает (3.13).

Пусть имеет место b). Предположим, что (3.13) не выполняется. Тогда $\lambda^{0}=0 \forall \lambda \in \Lambda_{k-1}\left(x_{0}\right)$. Поэтому в силу теоремы $2.7 \forall x \in \operatorname{ker} F^{\prime}\left(x_{0}\right) \exists y^{*} \in$ $\mathscr{F}_{k-1}^{2}\left(x_{0}\right):\left\langle y^{*}, F^{\prime \prime}\left(x_{0}\right)[x, x]\right\rangle \geqslant 0$. В силу 2-нормальности отображения $F$ и теоремы отделимости для выпуклых множеств $\exists \bar{y} \in\left(\operatorname{im} F^{\prime}\left(x_{0}\right)\right)^{\perp}:\left\langle y^{*}, \bar{y}\right\rangle>0$ $\forall y^{*} \in \operatorname{conv} \mathscr{F}_{k-1}^{2}\left(x_{0}\right)$. В силу условия 2-регулярности отображения $F$ по направлению $h$ существует такое конечномерное подпространство $\widetilde{X}$, что $\widetilde{G}(h) \widetilde{X}=Y$. Для малых по модулю $\varepsilon$ рассмотрим систему уравнений

$$
F^{\prime}\left(x_{0}\right) x=0, \quad \pi F^{\prime \prime}\left(x_{0}\right)[x, x]+\varepsilon \bar{y}|x|^{2}=0
$$

относительно неизвестного $x \in \widetilde{X}$. При $\varepsilon=0$ точка $x=h$ является ее решением. Применяя к этой системе уравнений в нормальной точке $x=h$ классическую теорему о неявной функции, получаем, что при малых по модулю 
$\varepsilon$ уравнение $(3.14)$ имеет решение $h(\varepsilon)$, для которого $h(\varepsilon) \rightarrow h, \varepsilon \rightarrow 0$. Для каждого $\varepsilon$ в силу сказанного выше существует $y^{*}(\varepsilon) \in \mathscr{F}_{k-1}^{2}\left(x_{0}\right)$ такое, что $\left\langle y^{*}(\varepsilon), F^{\prime \prime}\left(x_{0}\right)[h(\varepsilon), h(\varepsilon)]\right\rangle \geqslant 0$. Умножая второе уравнение в (3.14) при $x=h(\varepsilon)$, $\varepsilon>0$, скалярно на $y^{*}(\varepsilon)$ и учитывая, что $\left\langle y^{*}(\varepsilon), \bar{y}\right\rangle>0$, получаем противоречие с предыдущим неравенством. Лемма доказана.

Лемма 3.2. Пусть точка $x_{0}$ анормальна для отображения $F$ и является точкой локального относительно конечной топологии минимума в задаче (3.1). Предположим, что выполняется хотя бы одно из следующих предположений:

с) для любого ненулевого $y^{*} \in\left(\operatorname{im} F^{\prime}\left(x_{0}\right)\right)^{\perp}$ индекс квадратичной формы $\left\langle y^{*}, F^{\prime \prime}\left(x_{0}\right)[x, x]\right\rangle$, рассматриваемой на подпространстве $\operatorname{ker} F^{\prime}\left(x_{0}\right)$, больше чем $\operatorname{codim}\left(\operatorname{im} F^{\prime}\left(x_{0}\right)\right)$ - 1, или, что то же самое, множество $\mathscr{F}_{k-1}^{2}\left(x_{0}\right)$ nyсто;

d) существует такой вектор $\bar{x} \in \operatorname{ker} F^{\prime}\left(x_{0}\right)$, что $\left\langle y^{*}, F^{\prime \prime}\left(x_{0}\right)[\bar{x}, \bar{x}]\right\rangle<0$ для всех $y^{*} \in \mathscr{F}_{k-1}^{2}\left(x_{0}\right)$.

Тогда в точке $x_{0}$ выполняется классический приниип Лагранжа (3.13).

ДокАЗАтельство. Предположим, что (3.13) не выполняется. Тогда $\lambda^{0}=0$ $\forall \lambda \in \Lambda_{k-1}\left(x_{0}\right)$. Поскольку точка $x_{0}$ анормальна, то в силу теоремы 2.7 множество $\Lambda_{k-1}\left(x_{0}\right)$ непусто. Следовательно, множество $\mathscr{F}_{k-1}^{2}\left(x_{0}\right)$ также непусто и, значит, предположение с) нарушается. Кроме того, в силу теоремы 2.7 существует $y^{*} \in \mathscr{F}_{k-1}^{2}\left(x_{0}\right)$ такое, что $\left\langle y^{*}, F^{\prime \prime}\left(x_{0}\right)[\bar{x}, \bar{x}]\right\rangle \geqslant 0$. Значит, предположение d) также нарушается. Полученное противоречие завершает рассуждения. Лемма доказана.

Задачи с незамкнутым образом. Изначально теоремы 3.1 и 3.2 были доказаны в [20], когда $X$ и $Y$ - банаховы пространства, в предположении, что подпространство im $F^{\prime}\left(x_{0}\right)$ замкнуто. Позднее в [22] эти теоремы были обобщены на случай, когда подпространство im $F^{\prime}\left(x_{0}\right)$ априори замкнутым не является (при этом, естественно, банахово пространство $Y$ бесконечномерно). Приведем некоторые из этих результатов.

Будем предполагать, что отображения $f_{0}$ и $F$ дважды непрерывно дифференцируемы по Фреше в окрестности $x_{0}$, причем вторая производная $F$ удовлетворяет условию Липшица. Как показывают простые примеры (см. [22; примеры 3,4$])$, если в точке локального минимума $x_{0}$ задачи $(3.1)$ подпространство $\operatorname{im} F^{\prime}\left(x_{0}\right)$ не замкнуто, то в ней принцип Лагранжа (1.3) может нарушаться и, более того, может нарушаться даже необходимое условие первого порядка $f_{0}^{\prime}\left(x_{0}\right) \in\left(\operatorname{ker} F^{\prime}\left(x_{0}\right)\right)^{\perp}$. Тем не менее справедлива следующая теорема.

ТеОРема 3.5. Пусть $x_{0}$ - локальный минимум задачи (3.1). Тогда для любого

$$
h \in X: \quad F^{\prime}\left(x_{0}\right) h=0, \quad F^{\prime \prime}\left(x_{0}\right)[h, h] \in \operatorname{cl}\left(\operatorname{im} F^{\prime}\left(x_{0}\right)\right),
$$

для которого подпространство $\mathrm{im} G\left(x_{0}, h\right)$ замкнуто, существуют не равные одновременно нулю (зависящие от $h) \lambda^{0} \geqslant 0, y^{*} \in Y^{*}$, удовлетворяющие условиям

$$
\frac{\partial \mathscr{L}_{h}}{\partial x}\left(x_{0}, \lambda^{0}, y^{*}\right) \in\left(\operatorname{ker} F^{\prime}\left(x_{0}\right)\right)^{\perp}, \quad F^{\prime}\left(x_{0}\right)^{*} y^{*}=0
$$


Если же, кроме того, $\operatorname{im} G\left(x_{0}, h\right)=Y$ (т.е. отображение $F$ является 2-регулярным в точке $x_{0}$ по направлению $\left.h\right)$, то $\lambda^{0}>0$.

Здесь $\mathscr{L}_{h}$ - семейство функиий, зависящих от параметра $h \in X$ и переменных $\left(x, \lambda^{0}, y^{*}\right) \in X \times \mathbb{R} \times Y^{*}$, определенных по формуле

$$
\mathscr{L}_{h}\left(x, \lambda^{0}, y^{*}\right)=\lambda^{0} f_{0}(x)+\left\langle y^{*}, F^{\prime}(x) h\right\rangle .
$$

ТеОРема 3.6. Пусть $x_{0}$ - локалъный минимум задачи (3.1), образ $\operatorname{im} F^{\prime}\left(x_{0}\right)$ всюоду плотен в $Y$ и для некоторого $h \in \operatorname{ker} F^{\prime}\left(x_{0}\right)$ имеет место im $G\left(x_{0}, h\right)=Y$.

Тогда существует нормальный множитель Лагранжа $\lambda=\left(1, y^{*}\right), y^{*} \in Y^{*}$, для которого выполняется классический приниип Лагранжа (3.13) и, кроме mого,

$$
\frac{\partial^{2} L}{\partial x^{2}}\left(x_{0}, \lambda\right)[h, h] \geqslant 0 .
$$

Доказательства обеих теорем приведены в [22]. Эти результаты обобщены в [23] на задачу (2.13), где $C$ - произвольное выпуклое замкнутое подмножество банахова пространства $Y$. При этом в необходимых условиях второго порядка для задачи (2.13), так же как и в (2.16), появляется "сигма-член".

В настоящее время взаимосвязь двух изложенных подходов к исследованию необходимых условий экстремума для анормальных задач: индексного и основанного на обобщенной функции Лагранжа $\mathscr{L}_{\mathscr{A}}$ и понятии 2-регулярности изучена слабо. По существу, лишь в силу недавних результатов, полученных в [24], если в точке $x_{0}$ отображение $F$ не имеет 2-регулярных направлений, то для задачи (3.1) выполняются условия

$$
\begin{gathered}
\max _{\lambda \in \Lambda} \frac{\partial^{2} L}{\partial x^{2}}\left(x_{0}, \lambda\right)[x, x] \geqslant 0 \quad \forall x \in \operatorname{ker} F^{\prime}\left(x_{0}\right), \\
\text { где } \Lambda=\left\{\lambda \in \Lambda_{k-1}\left(x_{0}\right): \lambda^{0}=0\right\},
\end{gathered}
$$

даже более сильные, чем необходимые условия (2.4).

\section{4. Достаточные условия экстремума второго порядка}

Достаточные условия в терминах функции Лагранжа. Будем рассматривать задачу (2.1). Два предыдущих раздела посвящены необходимым условиям второго порядка. Займемся достаточными условиями второго порядка, предполагая, что $X, Y$ - нормированные пространства. Многое зависит от того, являются пространства $X$ и $Y$ конечномерными или нет. Будем предполагать, что все функции $f_{j}$ и отображение $f$ дважды непрерывно дифференцируемы по Фреше в окрестности точки $x_{0}$. Если оба пространства $X$ и $Y$ конечномерны, то для задачи (2.1) достаточные условия второго порядка в точке $x_{0}$ известны и заключаются в том, что

$$
\Lambda=\Lambda\left(x_{0}\right) \neq \varnothing, \quad \max _{\lambda \in \Lambda} \frac{\partial^{2} L}{\partial x^{2}}\left(x_{0}, \lambda\right)[x, x]>0 \quad \forall x \in \mathscr{K}\left(x_{0}\right): x \neq 0 .
$$

Пусть пространство $X$ бесконечномерно, а $Y$ конечномерно. Тогда (4.1) дает лишь достаточные условия локального минимума относительно конечной топологии, однако не является достаточным условием локального минимума относительно истинной топологии пространства $X$, даже в отсутствие ограничений 
(пример: $\left.X=l_{2}, f_{0}(x)=\sum_{i=1}^{\infty}\left(i^{-1} x_{i}^{2}-x_{i}^{4}\right), x_{0}=0\right)$. Далее будем рассматривать лишь локальный минимум относительно истинной топологии пространства $X$.

ТеОРема 4.1. Пусть пространство $Y$ конечномерно, множество $\Lambda=\Lambda\left(x_{0}\right)$ непусто и существует $\varepsilon>0$ такое, что

$$
\sup _{\lambda \in \Lambda} \frac{\partial^{2} L}{\partial x^{2}}\left(x_{0}, \lambda\right)[x, x] \geqslant \varepsilon\|x\|^{2} \quad \forall x \in \mathscr{K}\left(x_{0}\right) .
$$

Тогда точка $x_{0}$ является строгим локальным минимумом в задаче (2.1).

Эта теорема доказана в [5] (см. §1.7, теорема 7.1).

Условие (4.2) приводит к следующей проблеме, а именно, пусть пространство $X$ полно и, для простоты, ограничения типа неравенств отсутствуют, а точка $x_{0}$ нормальна. Тогда (4.2) принимает вид $\frac{\partial^{2} L}{\partial x^{2}}\left(x_{0}, \lambda\right)[x, x] \geqslant \varepsilon\|x\|^{2}$ $\forall x \in \operatorname{ker} f^{\prime}\left(x_{0}\right)$, откуда вытекает (см. [1; п. 7.2.2, с. 307, 308]), что подпространство ker $f^{\prime}\left(x_{0}\right)$ гомеоморфно гильбертову пространству. А это существенный недостаток достаточного условия (4.2), которое по существу применимо лишь в гильбертовых пространствах, тогда как в приложениях к бесконечномерным задачам чаще всего ни $X$, ни ker $f^{\prime}\left(x_{0}\right)$ гильбертовым не является. Например, в нелинейной задаче оптимального управления минимизация осуществляется на банаховом пространстве измеримых существенно ограниченных функций $L_{\infty}\left[t_{1}, t_{2}\right]$.

Если пространство $Y$ бесконечномерно, то, как доказано ниже, теорема 4.1 справедлива в предположении, что пространства $X, Y$ полны, а подпространство $\mathrm{im} f^{\prime}\left(x_{0}\right)$ замкнуто. Следующий пример показывает, что при этом предположение замкнутости $\operatorname{im} f^{\prime}\left(x_{0}\right)$ опустить нельзя.

Пример 4.1. Пусть $X=Y=l_{2}$ - гильбертовы пространства. Определим линейный компактный оператор $A: l_{2} \rightarrow l_{2}$ и квадратичное отображение $Q$ : $l_{2} \rightarrow l_{2}$ по формулам: $A x=\left(x_{1}, x_{2} / 2, \ldots, x_{j} / j, \ldots\right)$ и $Q(x)=\left(x_{1}^{2}, x_{2}^{2}, \ldots, x_{j}^{2}, \ldots\right)$. Рассмотрим задачу

$$
f_{0}(x)=-|x|^{2} \rightarrow \min , \quad f(x)=A x-Q(x)=0 .
$$

В этой задаче при $x_{0}=0$ условие $(4.2)$ выполнено, так как $(1,0) \in \Lambda(0)$, и по построению $\operatorname{ker} f^{\prime}\left(x_{0}\right)=\operatorname{ker} A=\{0\}$, однако $x_{0}=0$ локальным минимумом не является.

Действительно, обозначив через $x_{i} \in l_{2}$ последовательность, у которой на $i$-м месте стоит $i^{-1}$, а на остальных нули, имеем $f\left(x_{i}\right)=0, f_{0}\left(x_{i}\right)<0 \forall i, x_{i} \rightarrow 0$, $i \rightarrow \infty$. Здесь дело в том, что в этом примере подпространство $\operatorname{im} f^{\prime}\left(x_{0}\right)$ не замкнуто.

Задачи с бесконечномерным образом. Для бесконечномерных $Y$ предположение замкнутости $\operatorname{im} f^{\prime}\left(x_{0}\right)$ весьма обременительно. Наша первая цель привести достаточные условия второго порядка, свободные от этого предположения и от предположения о том, что подпространство ker $f^{\prime}\left(x_{0}\right)$ гомеоморфно гильбертову пространству. 
Воспользуемся конструкцией, предложенной в [25]. Зафиксируем точку $x_{0} \in X$. Пусть дана последовательность подмножеств $\left\{D_{i}\right\}$ из $X$ такая, что $x_{0} \in D_{i} \forall i$ и $\left\{D_{i}\right\}$ сходится к $x_{0}$ в том смысле, что любая последовательность точек $\left\{x_{i}\right\}$, лежащих в $D_{i}$, сходится к $x_{0}$. В качестве $D_{i}$ можно, например, взять шары $\left\{x:\left\|x-x_{0}\right\|_{1} \leqslant \rho_{i}\right\}$, где $\rho_{i} \rightarrow 0+$, а $\|\cdot\|_{1}-$ какая-нибудь норма в $X$, которой подчинена исходная норма.

Будем предполагать, что отображение $f$ является тейлоровским второго порядка в точке $x_{0}$ относительно $\left\{D_{i}\right\}$, т. е. существуют непрерывный линейный оператор $A: X \rightarrow Y$, непрерывное билинейное симметричное отображение $B: X \times X \rightarrow Y$ и числовая последовательность $\left\{\alpha_{i}\right\} \rightarrow 0+$ такие, что для каждого $i$ справедливо представление

$$
\begin{gathered}
f(x)=f\left(x_{0}\right)+A\left(x-x_{0}\right)+\frac{1}{2} B\left[x-x_{0}, x-x_{0}\right]+\Delta\left(x-x_{0}\right), \\
\left\|\Delta\left(x-x_{0}\right)\right\| \leqslant \alpha_{i}\left\|x-x_{0}\right\|^{2} \quad \forall x \in D_{i} .
\end{gathered}
$$

При этом нормы в пространствах $X$ и $Y$ для удобства обозначаются одинаково: через $\|\cdot\|$. Линейный оператор $A$, называемый относительным первым дифференциалом, будем обозначать через $\frac{\partial f}{\partial x}\left(x_{0}\right)$, а билинейную форму $B$, называемую относительным вторым дифференциалом, - через $\frac{\partial^{2} f}{\partial x^{2}}\left(x_{0}\right)$. Отметим, что так введенные относительные дифференциалы $A, B$, вообще говоря, определены неоднозначно (все зависит от выбора последовательности множеств $\left.\left\{D_{i}\right\}\right)$.

Функции $f_{j}$ также будем предполагать тейлоровскими второго порядка в точке $x_{0}$ относительно $\left\{D_{i}\right\}$ и их относительные дифференциалы будем обозначать аналогично. Эти же обозначения используются для частных производных функции Лагранжа по переменной $x$. Отметим, что даже в конечномерном случае и при $D_{i}=\left\{x:\left\|x-x_{0}\right\| \leqslant i^{-1}\right\}$ функция, являющаяся тейлоровской второго порядка в заданной точке, не обязана быть даже непрерывной в ее окрестности.

Далее для удобства снова будем считать, что $f_{j}\left(x_{0}\right)=0 \forall j \geqslant 1$. Положим

$$
\mathscr{K}_{1}\left(x_{0}\right)=\left\{x \in X:\left\langle\frac{\partial f_{j}}{\partial x}\left(x_{0}\right), x\right\rangle \leqslant 0, j=\overline{0, l}\right\} .
$$

ТЕОРема 4.2. Пусть множество $\Lambda=\Lambda\left(x_{0}\right)$ непусто и существуют $\gamma$, $\varepsilon>0$ maкuе, что

$$
\sup _{\lambda \in \Lambda} \frac{\partial^{2} L}{\partial x^{2}}\left(x_{0}, \lambda\right)[x, x]+\gamma\left\|\frac{\partial f}{\partial x}\left(x_{0}\right) x\right\|^{2} \geqslant \varepsilon\|x\|^{2} \quad \forall x \in \mathscr{K}_{1}\left(x_{0}\right) .
$$

Тогда для некоторого $i_{0}$ точка $x_{0}$ является строгим минимумом в задаче $(2.1)$ на множестве $D_{i_{0}}$, m.e.

$$
f_{0}(x)>f_{0}\left(x_{0}\right) \forall x \neq x_{0}: x \in D_{i_{0}}, f_{j}(x) \leqslant 0, j=\overline{1, l}, f(x)=0 .
$$


ДокАЗАтЕльство. Пусть, для удобства, $x_{0}=0, f_{0}\left(x_{0}\right)=0$. По лемме Хоффмана $[3]^{5}$

$$
\exists c_{1}>0: \operatorname{dist}\left(x, \mathscr{K}_{1}\left(x_{0}\right)\right) \leqslant c_{1} \sum_{j=0}^{l} \max \left(\left\langle\frac{\partial f_{j}}{\partial x}\left(x_{0}\right), x\right\rangle, 0\right) \quad \forall x .
$$

Доказательство теоремы проведем от противного, т. е. предположим, что существует последовательность $\left\{x_{i}\right\}$, для которой

$$
f\left(x_{i}\right)=0, \quad f_{j}\left(x_{i}\right) \leqslant 0, \quad j=\overline{0, l}, \quad x_{i} \in D_{i}, \quad x_{i} \neq 0 \quad \forall i .
$$

Поскольку $\left\{x_{i}\right\}$ сходится к нулю, то из представления для $f$ и $f_{j}$ имеем:

$$
\left\|\frac{\partial f}{\partial x}\left(x_{0}\right) x_{i}\right\|=o\left(\left\|x_{i}\right\|\right), \quad\left\langle\frac{\partial f_{j}}{\partial x}\left(x_{0}\right), x_{i}\right\rangle \leqslant o\left(\left\|x_{i}\right\|\right), \quad j=\overline{0, l} .
$$

Следовательно, в силу (4.4) существует такая последовательность $\left\{\tilde{x}_{i}\right\}$, что $x_{i}=\tilde{x}_{i}+o\left(\left\|\tilde{x}_{i}\right\|\right), \tilde{x}_{i} \neq 0, \tilde{x}_{i} \in \mathscr{K}_{1}\left(x_{0}\right) \forall i$, где $o\left(\left\|\tilde{x}_{i}\right\|\right) /\left\|\tilde{x}_{i}\right\| \rightarrow 0, i \rightarrow \infty$. В силу (4.3) $\forall i \exists \lambda_{i}=\left(\lambda_{i}^{0}, \ldots, \lambda_{i}^{l}, y_{i}^{*}\right) \in \Lambda$ :

$$
\frac{\partial^{2} L}{\partial x^{2}}\left(x_{0}, \lambda_{i}\right)\left[\tilde{x}_{i}, \tilde{x}_{i}\right]+\gamma\left\|\frac{\partial f}{\partial x}\left(x_{0}\right) \tilde{x}_{i}\right\|^{2} \geqslant \frac{\varepsilon}{2}\left\|\tilde{x}_{i}\right\|^{2} .
$$

Имеем

$$
\begin{aligned}
0 \stackrel{(1)}{\geqslant} & \lambda_{i}^{0} f_{0}\left(x_{i}\right) \stackrel{(2)}{\geqslant} L\left(x_{i}, \lambda_{i}\right) \stackrel{(3)}{=} L\left(x_{0}, \lambda_{i}\right)+\frac{\partial L}{\partial x}\left(x_{0}, \lambda_{i}\right) x_{i} \\
& +\frac{1}{2} \frac{\partial^{2} L}{\partial x^{2}}\left(x_{0}, \lambda_{i}\right)\left[x_{i}, x_{i}\right]+o\left(\left\|x_{i}\right\|^{2}\right) \stackrel{(4)}{=} \frac{1}{2} \frac{\partial^{2} L}{\partial x^{2}}\left(x_{0}, \lambda_{i}\right)\left[\tilde{x}_{i}, \tilde{x}_{i}\right]+o\left(\left\|\tilde{x}_{i}\right\|^{2}\right) \\
\stackrel{(5)}{=} & \frac{1}{2}\left(\frac{\partial^{2} L}{\partial x^{2}}\left(x_{0}, \lambda_{i}\right)\left[\tilde{x}_{i}, \tilde{x}_{i}\right]+\gamma\left\|\frac{\partial f}{\partial x}\left(x_{0}\right) \tilde{x}_{i}\right\|^{2}\right)+o\left(\left\|\tilde{x}_{i}\right\|^{2}\right) \stackrel{(6)}{\geqslant} \frac{\varepsilon}{4}\left\|\tilde{x}_{i}\right\|^{2}+o\left(\left\|\tilde{x}_{i}\right\|^{2}\right) .
\end{aligned}
$$

(1) (2)

Здесь неравенства $\geqslant$ и $\geqslant$ вытекают из предположения (4.5) и неотрицательности всех $\lambda_{i}^{j}$, равенство $\stackrel{(3)}{=}$ вытекает из представления для $f, f_{j}$ и ограниченности множества $\Lambda, \stackrel{(4)}{=}$ следует из того, что $\lambda_{i} \in \Lambda$, и построения последовательности $\left\{\tilde{x}_{i}\right\}, \stackrel{(5)}{=}$ вытекает из того, что в силу (4.6) $\left\|\frac{\partial f}{\partial x}\left(x_{0}\right) x_{i}\right\|^{2}=o\left(\left\|x_{i}\right\|^{2}\right)$, а неравенство $\stackrel{(6)}{\geqslant}-$ из (4.7). Таким образом, имеем $0 \geqslant \varepsilon\left\|x_{i}\right\|^{2} / 4+o\left(\left\|x_{i}\right\|^{2}\right)$. Полученное противоречие доказывает теорему.

ЗАмечание. На самом деле, доказано большее. А именно, $f_{0}(x) \geqslant f_{0}\left(x_{0}\right)+$ $\varepsilon\left\|x-x_{0}\right\|^{2} / 4$ для всех $x$, лежащих в $D_{i_{0}}$ и удовлетворяющих ограничениям задачи (2.1).

\footnotetext{
${ }^{5}$ Хотя лемма Хоффмана обычно формулируется в предположении, что $X$ полно, тем не менее из доказательства, приведенного в [3], непосредственно вытекает, что для рассматриваемого конечногранного конуса $\mathscr{K}_{1}\left(x_{0}\right)$ она справедлива и в произвольном нормированном пространстве $X$.
} 
Обсудим доказанную теорему. Случай $D_{i}=\left\{x:\left\|x-x_{0}\right\| \leqslant i^{-1}\right\}$ соответствует локальному минимуму задачи (2.1). Сравним достаточные условия (4.2) и (4.3). Очевидно, из (4.3) всегда вытекает (4.2). Оказывается, что при дополнительном предположении, что пространства $X, Y$ банаховы, а подпространство $\mathrm{im} f^{\prime}\left(x_{0}\right)$ замкнуто, из (4.2) следует (4.3). Это вытекает из следующего обобщения теоремы Финслера [26].

Пусть задан линейный непрерывный оператор $A: X \rightarrow Y$ и выполняется хотя бы одно из двух предположений: либо $Y$ конечномерно, либо пространства $X, Y$ полны и $\operatorname{im} A$ замкнут. Пусть дано семейство билинейных симметричных отображений $B_{\sigma}(\cdot, \cdot): X \times X \rightarrow \mathbb{R}$, зависящее от параметра $\sigma$, принимающего значения в заданном множестве $\Sigma$. Будем предполагать, что семейство $B_{\sigma}$ ограничено равномерно по $\sigma$, т. е. $\exists c_{2}>0:\left|B_{\sigma}\left(x_{1}, x_{2}\right)\right| \leqslant c_{2}\left\|x_{1}\right\|\left\|x_{2}\right\|$ $\forall \sigma \in \Sigma$. Положим $B_{\sigma}(x)=B_{\sigma}(x, x)$. Рассмотрим также конечногранный конус $K=\left\{x \in X:\left\langle a_{j}, x\right\rangle \leqslant 0, j=\overline{1, k}\right\}$, где $a_{j} \in X^{*}$ заданы.

Лемма 4.1. Предположим, что для некоторого $\varepsilon>0$ имеет место

$$
\sup _{\sigma \in \Sigma} B_{\sigma}(x) \geqslant \varepsilon\|x\|^{2} \quad \forall x \in \operatorname{ker} A \cap K .
$$

Тогда

$$
\exists \gamma \geqslant 0: \quad \sup _{\sigma \in \Sigma} B_{\sigma}(x)+\gamma\|A x\|^{2} \geqslant \frac{\varepsilon}{4}\|x\|^{2} \quad \forall x \in K
$$

ДокаЗАтельство. Рассмотрим конус $\widetilde{K}=\{x \in K: A x=0\}$. Оценивая расстояние от точки $x \in K$ до конуса $\widetilde{K}$, в силу леммы Хоффмана получаем существование такого $c_{3}>0$, что произвольный вектор $x \in K$ можно представить в виде $x=a+b$, где $a \in K, A a=0, c_{3}\|b\| \leqslant\|A b\|$.

Возьмем произвольный $x \in K$. Используя это представление, при $\gamma>0$ имеем

$$
\begin{aligned}
\sup _{\sigma \in \Sigma} & B_{\sigma}(x)+\gamma\|A x\|^{2}=\sup _{\sigma \in \Sigma} B_{\sigma}(a+b, a+b)+\gamma\|A b\|^{2} \\
& \geqslant \sup _{\sigma \in \Sigma} B_{\sigma}(a)-2 c_{2}\|a\|\|b\|-c_{2}\|b\|^{2}+\gamma c_{3}^{2}\|b\|^{2} \\
& \geqslant \varepsilon\|a\|^{2}-2 c_{2}\|a\|\|b\|+\left(\gamma c_{3}^{2}-c_{2}\right)\|b\|^{2} .
\end{aligned}
$$

Выберем $\gamma>0$ так, чтобы выражение, стоящее в правой части полученного неравенства, оценивалось снизу величиной $\varepsilon\left(\|a\|^{2}+\|b\|^{2}\right) / 2$. Учитывая, что $\|a\|^{2}+\|b\|^{2} \geqslant\|x\|^{2} / 2$, завершаем доказательство леммы.

Из этой леммы вытекает, что если либо пространство $Y$ конечномерно, либо пространства $X, Y$ банаховы и подпространство $\operatorname{im} f^{\prime}\left(x_{0}\right)$ замкнуто, то достаточные условия (4.2) и (4.3) эквивалентны. В то же время, как показывает приведенный выше пример, без предположения замкнутости $\mathrm{im} f^{\prime}\left(x_{0}\right)$ соотношение (4.2) достаточным условием локального минимума не является. Приведем пример, в котором подпространство im $f^{\prime}\left(x_{0}\right)$ замкнутым не является, но предположения теоремы 4.2 выполняются. Рассмотрим задачу

$$
f_{0}(x)=|x|^{2}-|\alpha A x|^{2}+r(x) \rightarrow \min , \quad f(x)=A x-Q(x)+R(x)=0 .
$$


Здесь $X=Y=l_{2}$, линейный оператор $A$ и квадратичное отображение $Q$ определены в примере 4.1, $\alpha$ - произвольная константа, а $r, R$ - произвольные гладкие отображения, у которых первые две производные в нуле равны нулю. Очевидно, что в точке $x_{0}=0$ выполнены все предположения теоремы 4.2 и, значит, $x_{0}=0$ является локальным минимумом, хотя $\operatorname{im} f^{\prime}\left(x_{0}\right)=\operatorname{im} A$ не замкнуто.

Нелинейные задачи оптимального управления естественно ставить как задачи минимизации в пространстве $X=L_{\infty}=L_{\infty}\left[t_{1}, t_{2}\right]$. А в нем (4.2) не выполняется. Если же $L_{\infty}$ наделить нормой из $L_{2}$, то оно перестанет быть полным и теорему 4.1 снова применять нельзя. Теорема же 4.2 не предполагает полноты $X$ и поэтому применима.

Поясним сказанное, рассмотрев задачу Лагранжа

$$
\dot{x}=f(x, u, t), \quad t \in\left[t_{1}, t_{2}\right], \quad \int_{t_{1}}^{t_{2}} f_{0}(x, u, t) d t \rightarrow \min
$$

с фиксированным левым концом $x\left(t_{1}\right)$ и ограничениями типа равенств и неравенств для правого конца $x\left(t_{2}\right)$. В качестве $X$ возьмем евклидово пространство измеримых ограниченных функций $u(\cdot)$, определенных на заданном отрезке $\left[t_{1}, t_{2}\right]$, наделив $X$ скалярным произведением из $L_{2}\left[t_{1}, t_{2}\right]$, порождающим норму $\|\cdot\|$, а в качестве $Y$ - конечномерное пространство, размерность которого определяется количеством концевых ограничений. В качестве $D_{i}$ возьмем шары $D_{i}=\left\{u:\left\|u-u_{*}\right\|_{L_{\infty}} \leqslant i^{-1}\right\}$, где $u_{*}-$ исследуемое на минимум допустимое управление. Непосредственно проверяется, что если вектор-функция $f$ и функция $f_{0}$ дважды непрерывно дифференцируемы по конечномерным переменным $(x, u)$, то теорема 4.2 применима и дает достаточные условия слабого локального минимума в задаче (4.8).

Достаточные условия для анормальных задач. Достаточные условия из теорем 4.1, 4.2, сформулированные в терминах классической функции Лагранжа $L$, справедливы без априорных предположений регулярности. Однако, если точка $x_{0}$ анормальна, то эти достаточные условия не могут выполняться для целых классов задач. Поясним сказанное, при этом для простоты ограничившись рассмотрением исследованной в предыдущем разделе задачи (3.1) и предполагая, что пространство $X$ является банаховым, пространство $Y$ - конечномерным, функция $f_{0}$ дважды, а отображение $F$ трижды непрерывно дифференцируемы в окрестности точки $x_{0}$, причем третья производная $F$ удовлетворяет в этой окрестности условию Липшица.

Предположим, что в анормальной точке $x_{0}$, являющейся решением задачи (3.1), классическое правило множителей Лагранжа (3.13) не выполняется, т. е. $\nexists y^{*} \in Y^{*} f_{0}^{\prime}\left(x_{0}\right)+F^{\prime}\left(x_{0}\right)^{*} y^{*}=0$, а также конус $H\left(x_{0}\right)$ является ненулевым. (Если $X$ конечномерно и $x_{0}$ не является изолированной точкой множества $\{x: F(x)=0\}$, то этот конус ненулевой.) Тогда предположения теорем 4.1, 4.2 выполняться не могут. Это вытекает из того, что в описанном случае

$$
\frac{\partial^{2} L}{\partial x^{2}}\left(x_{0}, \lambda\right)[x, x]=0 \quad \forall \lambda \in \Lambda\left(x_{0}\right), \quad \forall x \in H\left(x_{0}\right) \subseteq \operatorname{Ker} F^{\prime}\left(x_{0}\right), \quad H\left(x_{0}\right) \neq\{0\} .
$$


Приведем в терминах обобщенной функции Лагранжа $\mathscr{L}_{\mathscr{A}}$ достаточные условия экстремума второго порядка, которые остаются содержательными и в описанной выше ситуации, но выполняются лишь при следующих условиях сильной 2-регулярности.

ОПРедЕлЕниЕ. Отображение $F$ называется сильно 2-регулярным в точке $x_{0}$, если существует такое $\varepsilon>0$, что

$$
\widetilde{G}(h)\left(B_{X}\right) \supseteq \varepsilon B_{Y} \quad \forall h \in X:\|h\|=1,\|\widetilde{G}(h) h\| \leqslant \varepsilon .
$$

Здесь $B_{X}$ и $B_{Y}$ - единичные шары в пространствах $X$ и $Y$ соответственно, $\widetilde{G}(h) x=F^{\prime}\left(x_{0}\right) x+\pi F^{\prime \prime}\left(x_{0}\right)[h, x]$, а $\pi$ - оператор ортогонального проектирования $Y$ на $\left(\operatorname{im} F^{\prime}\left(x_{0}\right)\right)^{\perp}$.

Отметим, что если $X$ конечномерно, то сильная 2-регулярность эквивалентна 2-регулярности. В бесконечномерных пространствах это уже не так, а критерии сильной 2-регулярности в гильбертовом пространстве приведены в [27], [28].

ТеОрема 4.3. Предположим, что отображение F является сильно 2-регулярным в точке $x_{0}$. Пусть существует такое $\delta>0$, что для любого $h \in$ $H\left(x_{0}\right) \backslash\{0\}$ найдутся множители Лагранжа $\lambda_{A}=\left(\lambda^{0}, y_{1}^{*}, y_{2}^{*}\right):\left|\lambda_{A}\right|=1$, удовлетворяющие $(3.3),(3.4)$, для которых

$$
\frac{\partial^{2} \mathscr{L}_{\mathscr{A}}}{\partial x^{2}}\left(x_{0}, \lambda^{0}, y_{1}^{*}, \frac{1}{3} y_{2}^{*}, h\right)[h, h]>\delta\|h\|^{2} .
$$

Тогда точка $x_{0}$ является строгим локальным минимумом в задаче (3.1).

Эта теорема доказана в [5; гл. $1, \S 1.14]$.

Из теоремы 4.3 вытекает, что если отображение $F$ сильно 2-регулярно в точке $x_{0}$ и $H\left(x_{0}\right)=\{0\}$, то $x_{0}$ является строгим локальным минимумом в задаче (3.1). Кроме того, если пространство $X$ конечномерно, то в (4.9) достаточно взять $\delta=0$. Приведем пример, в котором предположения теоремы 4.3 выполняются, а предположения теорем 4.1 и 4.2 - нет.

ПримеР 4.2. Пусть $k=2, x=\left(x_{1}, \ldots, x_{n}\right) \in \mathbb{R}^{n}=X$,

$$
\begin{gathered}
f_{0}(x)=x_{1}+\sum_{i=2}^{n} x_{i}^{2} \rightarrow \min \\
F_{1}(x)=x_{1}\left(x_{1}-2 x_{n}\right)=0, \quad F_{2}(x)=\frac{1}{2} x_{1}^{2}+\sum_{i=2}^{n-1} x_{i}^{2}-x_{n}^{2}=0 .
\end{gathered}
$$

Здесь $x_{0}=0$ удовлетворяет правилу множителей Лагранжа, однако любой множитель Лагранжа $\lambda \in \Lambda(0)$ имеет вид $\lambda=\left(0, y^{*}\right)$. Поэтому $\frac{\partial^{2} L}{\partial x^{2}}\left(x_{0}, \lambda\right)[h, h]=0$ $\forall h \in H\left(x_{0}\right)=\{x: F(x)=0\}, \forall \lambda \in \Lambda(0)$ и, значит, (4.2) нарушается.

С другой стороны, $h_{1}=0$ для любого $h \in H(0)$ и отображение $F$ является 2-регулярным в нуле. Поэтому для любого $\lambda_{A}$, удовлетворяющего $(3.3),(3.4)$, 
имеем:

$$
\begin{aligned}
\lambda^{0}>0, y_{1}^{*}=0 \Rightarrow \quad \forall h \in H(0) \backslash\{0\} \exists \lambda_{A}=\left(\lambda^{0}, 0, y_{2}^{*}\right): \\
\\
\quad \frac{\partial^{2} \mathscr{L}_{\mathscr{A}}}{\partial x^{2}}\left(0, \lambda^{0}, 0, \frac{1}{3} y_{2}^{*}, h\right)[h, h]=\lambda^{0} \sum_{i=2}^{n} h_{i}^{2}>0 .
\end{aligned}
$$

В отличие от теорем 4.1, 4.2, в теореме 4.3 предполагается сильная 2-регулярность отображения $F$. Следующий пример показывает существенность этого предположения.

ПримеР 4.3. Пусть $k=1, x=\left(x_{1}, x_{2}\right) \in \mathbb{R}^{2}=X$. В задаче

$$
f_{0}(x)=-|x|^{2} \rightarrow \min , \quad F(x)=x_{1}^{2}+x_{2}^{3}=0
$$

точка $x_{0}=0$, очевидно, минимумом не является. В то же время $H\left(x_{0}\right)=\{h=$ $\left.\left(h_{1}, h_{2}\right): h_{1}=0\right\}$ и, значит, (4.9) выполняется при $\lambda_{A}=(0,0,1), \delta=1$. Здесь все дело в том, что отображение $F$ не является 2-регулярным в нуле.

Достаточные условия высших порядков для задачи (2.1) получены в [5; гл. $1, \S 1.14]$, а достаточные условия второго порядка для задачи (2.13), где $C$ - выпуклое замкнутое множество, получены в [15].

\section{5. Квадратичные отображения и квадратичные задачи}

\section{Знакоопределенность квадратичной формы на пересечении квад-} рик. Типичный пример экстремальной задачи, в которой экстремум всегда достигается в анормальной точке, дает следующая классическая алгебраическая проблема. Даны $(k+1)$ квадратных симметричных $(n \times n)$-матриц $Q_{i}$, $i=\overline{0, k}$, определяющих на $X=\mathbb{R}^{n}$ квадратичные формы $q_{i}: q_{i}(x)=\left\langle Q_{i} x, x\right\rangle$. Требуется получить условия, при которых

$$
q_{0}(x) \geqslant 0 \quad \forall x: q_{1}(x)=q_{2}(x)=\cdots=q_{k}(x)=0 .
$$

Здесь $n, k$ - заданные натуральные числа. Эта задача представляет интерес как сама по себе, так и в применении к прикладным проблемам (см. [29]-[31] и т. д.) Очевидно, что (5.1) равносильно тому, что в задаче минимизации

$$
q_{0}(x) \rightarrow \min , \quad q_{i}(x)=0, \quad i=\overline{1, k},
$$

точка $x_{0}=0$ является минимумом. Однако эта точка автоматически анормальна.

Применяя к задаче (5.2) необходимые условия второго порядка (2.18) из теоремы 2.7, получаем, что если выполняется (5.1), то

$$
\Lambda_{k-1} \neq\{\varnothing\}, \quad \max _{\lambda \in \Lambda_{k-1}}\left(\lambda^{0} q_{0}(x)+\sum_{j=1}^{k} y_{j}^{*} q_{j}(x)\right) \geqslant 0 \quad \forall x \in X .
$$

Здесь

$$
\Lambda_{s}=\left\{\lambda=\left(\lambda^{0}, y^{*}\right): \lambda^{0} \geqslant 0, \text { ind }\left(\lambda^{0} q_{0}+\sum_{j=1}^{k} y_{j}^{*} q_{j}\right) \leqslant s,|\lambda|=1\right\} .
$$


Однако среди векторов $\lambda \in \Lambda_{k-1}$ могут оказаться и те, для которых $\lambda^{0}=0$. А это приводит к тому, что условия (5.3) перестают зависеть от формы $q_{0}$, что может сделать их неинформативными. Так обязательно произойдет, если $0 \in \operatorname{conv} \Lambda_{k-1}$, так как в этом случае, очевидно, (5.3) выполняется автоматически. Вот соответствующий пример: $k=2, q_{1}(x)=x_{1} x_{2}$, а формы $q_{0}$ и $q_{2}$ произвольны. Очевидно, conv $\Lambda_{1}$ содержит отрезок $\left(0, \lambda_{1}, 0\right),\left|\lambda_{1}\right| \leqslant 1$ и, значит, (5.3) выполняется для любых $q_{0}, q_{2}$ и, в частности, для тех, для которых (5.1) нарушается (например, для $q_{0}(x)=-|x|^{2}, q_{2}=0$ ).

Наша ближайшая цель - найти условия, гарантирующие, что если выполняется (5.1), то в (5.3) можно ограничиться лишь теми $\lambda$, для которых $\lambda^{0}>0$. Следующий очевидный пример показывает, что без дополнительных условий это не так и, более того, может оказаться, что $\lambda^{0}=0 \forall \lambda \in \Lambda_{k-1}$. Действительно, пусть $k=1, n=2, q_{0}(x)=x_{1} x_{2}, q_{1}(x)=x_{1}^{2}$. Очевидно, $q_{0}(x)=0$ $\forall x: q_{1}(x)=0$, однако $\lambda^{0}=0 \forall \lambda \in \Lambda_{0}$.

Введем нужные в дальнейшем обозначения. Определим билинейное отображение $\widetilde{Q}: X \times X \rightarrow Y=\mathbb{R}^{k}$, которое произвольным $x_{1}, x_{2} \in X$ ставит в соответствие вектор $y=\widetilde{Q}\left[x_{1}, x_{2}\right]$, $i$-я координата которого равна $y_{i}=\left\langle Q_{i} x_{1}, x_{2}\right\rangle$. В силу симметричности матриц $Q_{i}$ так определенное билинейное отображение $\widetilde{Q}$ также симметрично. Положим $Q(x)=\widetilde{Q}[x, x]$. Отображение $Q: X \rightarrow Y$ называется квадратичным. Для фиксированного $h \in X$ обозначим через $Q h$ линейный оператор, действующий из $X$ в $Y$ по формуле $Q h(x)=\widetilde{Q}[h, x]$. Для $y^{*} \in Y^{*}$ положим

$$
y^{*} Q=\sum_{i=1}^{k} y_{i}^{*} Q_{i}, \quad y^{*} Q(x)=\sum_{i=1}^{k} y_{i}^{*} q_{i}(x) .
$$

Пусть в $Y$ задан выпуклый замкнутый конус $C$. Рассмотрим конус

$$
K=\{x \in X: Q(x) \in C, x \neq 0\} .
$$

ОПРЕДЕЛЕНИЕ. Квадратичное отображение $Q$ называется регулярным относительно конуса $C$ в точке $h \in K$, если

$$
\operatorname{im} Q h-C=Y \text {. }
$$

Квадратичное отображение $Q$ называется регулярным относительно конуса $C$, если оно регулярно в каждой точке $h \in K$. Для $C=\{0\}$ точки конуса $K$ называются нетривиальными нулями квадратичного отображения $Q$. При этом регулярность $Q$ в точке $h: Q(h)=0$ означает линейную независимость векторов $Q_{1} h, \ldots, Q_{k} h$, и эту точку $h$ называют регулярным нулем, а регулярность $Q$ означает регулярность всех его нетривиальных нулей (если они существуют).

Квадратичное отображение $Q$ называется сюръективным, если $Q(X)=Y$. Если у $Q$ существует регулярный нуль $h$, то $Q$ сюръективно. Действительно, $Q(h)=0$ и векторы $Q_{1} h, \ldots, Q_{k} h$ линейно независимы. Поэтому из классической теоремы об обратной функции, примененной к уравнению $Q(x)=y$ в точке $x=h$, вытекает, что при отображении $Q$ образ единичной окрестности 
точки $h$ содержит некоторую окрестность нуля, откуда в силу положительной однородности отображения $Q$ следует его сюръективность.

Для $y \in(Q(X)-C)$ через $\omega(y)$ обозначим инфимум в задаче

$$
q_{0}(x) \rightarrow \inf , \quad Q(x)-y \in C,
$$

и положим $\omega(y)=+\infty \forall y \notin(Q(X)-C)$. Так определенная функция $\omega$ положительно однородна и если

$$
q_{0}(x) \geqslant 0 \quad \forall x: Q(x) \in C,
$$

то $\omega(0)=0$. Обозначим через $S=\{y \in Y:|y|=1\}$ единичную сферу.

Лемма 5.1. Пусть выполняется (5.5) u, кроме того, если $h \in K, q_{0}(h)=0$, то $Q$ регулярно относительно конуса $C$ в точке $h$. Тогда функиия $\omega$ ограничена снизу на $S$, m. е. существует $d>0$ такое, что $\omega(y) \geqslant-d \forall y \in S$.

ДокАзАтельство. Предположим противное. Тогда существуют такие последовательности $\left\{x_{i}\right\} \subset X,\left\{\tilde{\eta}_{i}\right\} \subset C$, что $Q\left(x_{i}\right)-\tilde{\eta}_{i}=y_{i} \in S \forall i, q_{0}\left(x_{i}\right) \rightarrow$ $-\infty \Rightarrow\left|x_{i}\right| \rightarrow \infty$. Положим $h_{i}=x_{i}\left|x_{i}\right|^{-1}, \eta_{i}=\tilde{\eta}_{i}\left|x_{i}\right|^{-2}$. Поскольку $\left|h_{i}\right|=1$, то, переходя к подпоследовательности, будем считать, что $h_{i} \rightarrow h$, откуда $\eta_{i} \rightarrow \eta \in C$ так как $\left|Q\left(h_{i}\right)-\eta_{i}\right|=\left|x_{i}\right|^{-2} \rightarrow 0$. Очевидно, $q_{0}(h) \leqslant 0$, а также $Q(h)=\eta \Rightarrow h \in K$. Следовательно, $q_{0}(h)=0$ и поэтому квадратичное отображение $Q$ регулярно относительно конуса $C$ в точке $h$ и, значит, оно удовлетворяет условию Робинсона. Поэтому по теореме Робинсона [16] в окрестности точки $h$ справедлива следующая оценка расстояния до множества $K$ : $\operatorname{dist}\left(h_{i}, K\right) \leqslant$ const $\times \operatorname{dist}\left(Q\left(h_{i}\right), C\right) \leqslant$ const $\left|y_{i}\right|\left|x_{i}\right|^{-2}=$ const $\left|x_{i}\right|^{-2}$, поскольку $Q\left(h_{i}\right)-y_{i}\left|x_{i}\right|^{-2}=\eta_{i} \in C$. Напомним, что dist - pacстояние от точки до множества, a const здесь и далее означает некоторые константы, конкретный вид которых нам не важен. Значит, для больших номеров $i$ существуют такие $\tilde{h}_{i} \in K, \xi_{i}$, что $h_{i}=\tilde{h}_{i}+\xi_{i},\left|\xi_{i}\right| \leqslant$ const $\left|x_{i}\right|^{-2}$. Тогда

$$
q_{0}\left(h_{i}\right)=q_{0}\left(\tilde{h}_{i}\right)+q_{0}\left(\xi_{i}\right)+2\left\langle Q_{0} \tilde{h}_{i}, \xi_{i}\right\rangle \geqslant- \text { const }\left|\xi_{i}\right| \geqslant- \text { const }\left|x_{i}\right|^{-2}
$$

(так как в силу $\left.(5.5) q_{0}\left(\tilde{h}_{i}\right) \geqslant 0\right)$, откуда имеем $q_{0}\left(x_{i}\right)=\left|x_{i}\right|^{2} q_{0}\left(h_{i}\right) \geqslant-$ const $\forall i$. Полученное противоречие завершает доказательство леммы.

СлЕДСтвиЕ. Пусть выполняются все предположения леммы 5.1. Тогда

$$
q_{0}(x)+d|Q(x)-\eta| \geqslant 0 \quad \forall x \in X, \quad \eta \in C,
$$

где константа $d>0$ взята из леммы.

Действительно, в силу положительной однородности функции $\omega$ имеем $\omega(y)+d|y| \geqslant 0 \forall y$. Пусть $\eta \in C, x \in X$. Подставляя в это неравенство $y=Q(x)-\eta$ и учитывая, что $q_{0}(x) \geqslant \omega(y)$, получаем (5.6).

Для $\varepsilon \geqslant 0$ и целых $s \geqslant 0$ положим

$$
\Lambda_{s}(C)=\left\{\lambda=\left(\lambda^{0}, y^{*}\right) \in \Lambda_{s}: y^{*} \in C^{0}\right\}, \quad \Lambda_{s}^{\varepsilon}(C)=\left\{\lambda \in \Lambda_{s}(C): \lambda^{0} \geqslant \varepsilon\right\},
$$

где $C^{0}=\left\{y^{*}:\left\langle y^{*}, y\right\rangle \leqslant 0 \forall y \in C\right\}$ - полярный конус к конусу $C$.

Далее будем предполагать, что конус $C$ является конечногранным, т. е. задается конечным числом линейных однородных неравенств. 
ТеОрема 5.1. Пусть выполняется (5.5) и, кроме того, если $h \in K, q_{0}(h)=0$, то $Q$ регулярно относительно конуса $C$ в точке $h$. Тогда существует $\varepsilon>0$ maкое, что

$$
\Lambda=\Lambda_{k-1}^{\varepsilon}(C) \neq \varnothing, \quad \max _{\lambda \in \Lambda}\left(\lambda^{0} \omega(y)+\left\langle y^{*}, y\right\rangle\right) \geqslant 0 \quad \forall y .
$$

Отметим, что случай $K=\varnothing$ не исключается. При этом для $y \notin(Q(X)-C)$ неравенство в (5.7) выполняется автоматически, так как для указанных $у$ имеем $\omega(y)=+\infty$.

Для доказательства теоремы нам потребуется следующая лемма.

Лемма 5.2. Пусть заданъ гладкие отображения $\varphi: X \rightarrow \mathbb{R}, \Phi: X \rightarrow Y u$ константа $d>0$. Предположим, что функиия $f(x, \eta)=\varphi(x)+d|\Phi(x)+\eta|$ достигает минимума по $x \in X, \eta \in C$ в точке $\left(x_{0}, \eta_{0}\right)$, для которой

$$
\Phi\left(x_{0}\right)+\eta_{0}=0, \quad \operatorname{im} \Phi^{\prime}\left(x_{0}\right)+\operatorname{span} \Gamma=Y, \quad \eta_{0} \in \operatorname{ri} \Gamma,
$$

где Г - некоторая грань конуса $C$, , ri - относительная внутренность выпуклого множества. Тогда существует $y^{*} \in C^{0}$ такое, что $\left|y^{*}\right| \leqslant d$,

$$
\varphi^{\prime}\left(x_{0}\right)+\Phi^{\prime}\left(x_{0}\right)^{*} y^{*}=0
$$

и для $q_{0}=\varphi^{\prime \prime}\left(x_{0}\right), Q=\Phi^{\prime \prime}\left(x_{0}\right)$ выполняется

$$
q_{0}(x)+y^{*} Q(x) \geqslant 0 \quad \forall x: \Phi^{\prime}\left(x_{0}\right) x \in \operatorname{span} \Gamma .
$$

Доказательство леммы аналогично доказательству предложения 2 из [32], в котором был рассмотрен случай $\Gamma=\{0\}$.

Докажем теорему 5.1. В силу положительной однородности функции $\omega$ достаточно доказать существование $\varepsilon>0$ такого, что множество $\Lambda=\Lambda_{k-1}^{\varepsilon}(C)$ непусто и неравенство в (5.7) выполняется для любых $y \in S$. Сделаем это.

По условию количество граней конуса $C$ конечно. Для каждой грани рассмотрим оператор ортогонального проектирования $Y$ на ортогональное дополнение к линейной оболочке этой грани. Отбросив нулевой оператор (если он есть) и перенумеровав оставшиеся операторы, будем считать, что это $P_{1}, \ldots, P_{r_{0}}$.

Зафиксируем произвольную точку $y \in S$. По теореме Сарда для каждого из гладких отображений $P_{r} Q: X \rightarrow \operatorname{im} P_{r}$ мера Лебега множества его критических значений равна нулю. Поэтому множество точек, которые являются регулярными значениями для каждого из отображений $P_{r} Q, r=\overline{1, r_{0}}$, всюду плотно в $Y$. Кроме того, каждое из этих отображений положительно однородно. Поэтому существует такая сходящаяся к $y$ последовательность $\left\{y_{s}\right\}$, что $y_{s} \in S \forall s$ и каждое $P_{r} y_{s}$ является регулярным значением всех отображений $P_{r} Q, r=\overline{1, r_{0}}$.

По лемме 5.1 существует $d>0$, для которого выполняется (5.6). Для натуральных $i$ положим $q_{0, i}(x)=q_{0}(x)+i^{-1}|x|^{2}, Q_{0, i}=Q_{0}+i^{-1} I$, где $I$ - единичная матрица. Зафиксируем произвольный номер $s$ и рассмотрим семейство задач

$$
f_{i}(x, \eta)=q_{0, i}(x)+d\left|Q(x)-\eta-y_{s}\right| \rightarrow \inf , \quad x \in X, \quad \eta \in C,
$$


зависящих от натурального параметра $i$. Они называются $i$-задачами. В силу леммы 5.1 инфимум в каждой из $i$-задач конечен. Докажем, что он достигается. Действительно, пусть $\left\{x_{j}, \eta_{j}\right\}$ - минимизирующая последовательность в $i$-задаче. Тогда в силу (5.6) выполнено неравенство $f_{i}\left(x_{j}, \eta_{j}\right) \geqslant i^{-1}\left|x_{j}\right|^{2}-d\left|y_{s}\right|$ и, значит, если $\left|x_{j}\right| \rightarrow \infty$, то $f_{i}\left(x_{j}, \eta_{j}\right) \rightarrow \infty, j \rightarrow \infty$, что невозможно. Поэтому последовательность $\left\{x_{j}\right\}$ ограничена, и, следовательно, $\left\{\eta_{j}\right\}$ также ограничена. Переходя к подпоследовательностям, добьемся того, что обе последовательности сходятся и, следовательно, их пределы дают решение $i$-задачи.

Через $x_{i, s}, \eta_{i, s}$ обозначим какое-нибудь решение $i$-задачи. Зафиксируем $i$ и рассмотрим два случая.

Первый случай: $Q\left(x_{i, s}\right)-\eta_{i, s} \neq y_{s}$. Тогда в $i$-задаче в окрестности решения $\left(x_{i, s}, \eta_{i, s}\right)$ минимизируемая функция $f_{i}$ является гладкой. Поэтому в силу классических необходимых условий первого и второго порядка имеем

$$
\begin{array}{cc}
\frac{\partial f_{i}}{\partial x}\left(x_{i, s}, \eta_{i, s}\right)=2\left(Q_{0, i}+y_{i, s}^{*} Q\right) x_{i, s} & =0, \quad \frac{\partial f_{i}}{\partial \eta}\left(x_{i, s}, \eta_{i, s}\right)=-y_{i, s}^{*} \in-C^{0}, \\
q_{0, i}(x)+y_{i, s}^{*} Q(x) \geqslant 0 & \forall x \in \operatorname{ker} Q x_{i, s},
\end{array}
$$

где $y_{i, s}^{*}=d\left(Q\left(x_{i, s}\right)-\eta_{i, s}-y_{s}\right) /\left|Q\left(x_{i, s}\right)-\eta_{i, s}-y_{s}\right|$. Проанализируем эти соотношения.

Пусть вначале $Q\left(x_{i, s}\right) \neq 0$. Тогда положим $\Pi_{i}=\operatorname{ker} Q x_{i, s}+\operatorname{span}\left\{x_{i, s}\right\}$. В силу $(5.10),(5.11)$ выполнено неравенство $q_{0, i}(x)+y_{i, s}^{*} Q(x) \geqslant 0 \forall x \in \Pi_{i}$. Кроме того, $\operatorname{codim} \Pi_{i} \leqslant k-1$, так как $x_{i, s} \notin \operatorname{ker} Q x_{i, s}$, поскольку $\left(Q x_{i, s}\right) x_{i, s}=Q\left(x_{i, s}\right) \neq 0$. Следовательно, учитывая (5.10), имеем

$$
\begin{array}{cl}
y_{i, s}^{*} \in C^{0}, \quad & \left|y_{i, s}^{*}\right| \leqslant d, \\
\lambda_{i, s}^{0}\left(1, y_{i, s}^{*}\right) \in \Lambda_{k-1} \quad \text { при } \quad \lambda_{i, s}^{0}=\left(1+\left|y_{i, s}^{*}\right|^{2}\right)^{-1 / 2} .
\end{array}
$$

Умножая первую формулу в (5.10) скалярно на $x_{i, s}$ и используя определение $y_{i, s}^{*}$, получаем

$$
q_{0, i}\left(x_{i, s}\right)+d\left|Q\left(x_{i, s}\right)-\eta_{i, s}-y_{s}\right|+\left\langle y_{i, s}^{*}, y_{s}\right\rangle=-\left\langle y_{i, s}^{*}, \eta_{i, s}\right\rangle \geqslant 0 .
$$

С другой стороны,

$q_{0, i}(x)+d\left|Q(x)-\eta-y_{s}\right| \geqslant q_{0, i}\left(x_{i, s}\right)+d\left|Q\left(x_{i, s}\right)-\eta_{i, s}-y_{s}\right| \quad \forall x \in X, \quad \forall \eta \in C$,

так как $\left(x_{i, s}, \eta_{i, s}\right)$ является решением $i$-задачи. Отсюда в силу предыдущего неравенства

$$
\min \left\{q_{0, i}(x)+d\left|Q(x)-\eta-y_{s}\right|, x \in X, \eta \in C\right\}+\left\langle y_{i, s}^{*}, y_{s}\right\rangle \geqslant 0 .
$$

Пусть $Q\left(x_{i, s}\right)=0$. Умножая (5.10) скалярно на $x_{i, s}$, получаем $q_{0, i}\left(x_{i, s}\right)=0$. Поэтому $\left(0, \eta_{i, s}\right)$ - также решение $i$-задачи. Подставляя в (5.11) вместо $x_{i, s}$ точку $x=0$, имеем $q_{0, i}(x)+y_{i, s}^{*} Q(x) \geqslant 0 \forall x$, откуда вытекает (5.12). Доказательство (5.14) аналогично.

Второй случай: $Q\left(x_{i, s}\right)-\eta_{i, s}=y_{s}$. Любая точка, принадлежащая конечногранному конусу, принадлежит относительной внутренности какой-нибудь из 
его граней. Поэтому у $C$ существует грань $\Gamma$, для которой $\eta_{i, s} \in \operatorname{ri} \Gamma$. Пусть вначале $\operatorname{span} \Gamma \neq Y$. Тогда, взяв соответствующий оператор проектирования $P_{r} \neq 0$ на $(\operatorname{span} \Gamma)^{\perp}$, имеем $P_{r} \eta_{i, s}=0 \Rightarrow P_{r} Q\left(x_{i, s}\right)=P_{r} y_{s}$. Следовательно, по построению $\operatorname{im} Q x_{i, s}+\operatorname{span} \Gamma=Y$ и, значит, для $i$-задачи выполнены предположения леммы 5.2. Из нее получаем существование $y_{i, s}^{*}$, удовлетворяющих $(5.10),(5.12)$. При этом мы использовали (5.9) и то, что коразмерность подпространства $\Pi_{i}=\left\{x: Q\left[x_{i, s}, x\right] \in \operatorname{span} \Gamma\right\}$ не превышает числа $(k-1)$. Если же $\operatorname{span} \Gamma=Y$, то $\eta_{i, s} \in \operatorname{int} C$, откуда легко вытекает, что условия (5.10), (5.12) выполняются при $y_{i, s}^{*}=0$.

Умножая первое равенство в (5.10) скалярно на $x_{i, s}$, имеем $q_{0, i}\left(x_{i, s}\right)+$ $\left\langle y_{i, s}^{*}, Q\left(x_{i, s}\right)\right\rangle=0$. Отсюда, используя (5.13) и учитывая, что имеют место соотношения $Q\left(x_{i, s}\right)-\eta_{i, s}-y_{s}=0,\left\langle y_{i, s}^{*}, \eta_{i, s}\right\rangle \leqslant 0$, получаем (5.14).

Таким образом, в результате рассмотрения двух указанных случаев доказано существование $y_{i, s}^{*}$, для которых имеют место (5.12), (5.14). Выделяя при каждом фиксированном $s$ из ограниченной последовательности $\left\{y_{i, s}^{*}\right\}$ сходящуюся подпоследовательность, добьемся того, что $y_{i, s}^{*} \rightarrow y_{s}^{*}$ при $i \rightarrow \infty$ для некоторого $y_{s}^{*}:\left|y_{s}^{*}\right| \leqslant d$. Аналогично, переходя от $\left\{y_{s}^{*}\right\}$ к подпоследовательности, добьемся того, что $y_{s}^{*} \rightarrow \hat{y}^{*}, s \rightarrow \infty$, для некоторого $\hat{y}^{*}$. Зафиксировав $x \in X, \eta \in C$ и переходя в (5.12) и (5.14) к пределу, вначале при фиксированном $s$ и $i \rightarrow \infty$, а затем при $s \rightarrow \infty$, в силу замкнутости множества $\Lambda_{k-1}$ имеем: $\left|\hat{y}^{*}\right| \leqslant d, \hat{y}^{*} \in C^{0}$,

$$
\begin{gathered}
\lambda=\left(\lambda^{0}, y^{*}\right) \in \Lambda_{k-1}(C) \quad \text { при } \quad \lambda^{0}=\left(1+\left|\hat{y}^{*}\right|^{2}\right)^{-1 / 2}, \quad y^{*}=\lambda^{0} \hat{y}^{*}, \\
\lambda^{0} \inf \left\{q_{0}(x)+d|Q(x)-\eta-y|, x \in X, \eta \in C\right\}+\left\langle y^{*}, y\right\rangle \geqslant 0 .
\end{gathered}
$$

Из последнего неравенства имеем $\lambda^{0} q_{0}(x)+\left\langle y^{*}, y\right\rangle \geqslant 0 \forall x: Q(x)-y \in C \Rightarrow$ $\lambda^{0} \omega(y)+\left\langle y^{*}, y\right\rangle \geqslant 0$. Положим $\varepsilon=\left(1+d^{2}\right)^{-1 / 2}$. Тогда $\lambda^{0} \geqslant \varepsilon$, так как $\left|\hat{y}^{*}\right| \leqslant d$. Теорема доказана.

Приведенное доказательство теоремы 5.1 основывается на исследовании задачи

$$
f(x, \eta)=q_{0}(x)+d|Q(x)-\eta-y| \rightarrow \inf , \quad x \in X, \quad \eta \in C,
$$

в которой инфимум в силу леммы 5.1 конечен для любого $y$. Если бы при каждом $y$ инфимум в (5.15) достигался, то в доказательстве теоремы не возникало бы необходимости рассмотрения семейства $i$-задач и было бы достаточно проанализировать точки минимума функции $f$. Если форма $q_{0}$ положительна на $K$, то, увеличив, если надо, $d$, из леммы 5.1 несложно получить, что любая минимизирующая последовательность задачи (5.15) ограничена и, значит, инфимум в ней достигается. Однако даже при $k=1$ без дополнительного предположения положительности $q_{0}$ на $K$ это уже не так. Действительно, пусть $C=\{0\}, q_{0}(x)=x_{1}^{2}, Q(x)=q_{1}(x)=x_{1} x_{2}$. Тогда для любых $d>0$ и $y \in Y \backslash\{0\}$ инфимум в (5.15) равен нулю, однако он не достигается.

Примеры, приведенные в [32], показывают, что даже при $C=\{0\}$ возможны следующие ситуации: выполнены все предположения теоремы 5.1 , однако инфимум в задаче (5.4) не достигается ни при каком $y \neq 0$; для любого $\alpha \in \mathbb{R}$ существует такое $y=y(\alpha)$, что в (5.4) инфимум равен $\alpha$, но не достигается; 
квадратичное отображение $Q$ сюръективно, и каждое $y \neq 0$ является для него регулярным значением, но все нетривиальные нули $Q$ нерегулярны и $q_{0}$ обращается на $K$ в нуль, в то же время $\omega(y)=-\infty \forall y \neq 0$.

Из теоремы 5.1 вытекает следующая теорема.

Tеорема 5.2. Пусть $q_{0}(x)>0 \forall x \in K$. Тогда существует $\varepsilon>0$ maкое, чmo

$$
\Lambda=\Lambda_{k-1}^{\varepsilon \varepsilon}(C) \neq \varnothing, \quad \max _{\lambda \in \Lambda}\left(\lambda^{0} q_{0}(x)+y^{*} Q(x)\right) \geqslant \varepsilon|x|^{2} \quad \forall x \in X .
$$

Здесъ множество $\Lambda_{s}^{\varepsilon \varepsilon}(C)$ состоит из $\lambda=\left(\lambda^{0}, y^{*}\right)$ maких, что $|\lambda|=1, \lambda^{0} \geqslant \varepsilon$, $y^{*} \in C^{0}$ и существует линейное подпространство

$$
\Pi \subset X: \quad \operatorname{codim} \Pi \leqslant s, \quad \lambda^{0} q_{0}(x)+y^{*} Q(x) \geqslant \varepsilon|x|^{2} \quad \forall x \in \Pi .
$$

Теоремы 5.1 и 5.2 дают необходимые условия неотрицательной и положительной определенности соответственно формы $q_{0}$ на конусе $K$. Достаточность их очевидна.

К сожалению, теоремы 5.1 и 5.2 существенно конечномерны и при $\operatorname{dim} X=\infty$ их утверждения могут не выполняться. Вот соответствующий пример.

ПримеР 5.1. Пусть $X$ - бесконечномерное гильбертово пространство, $Y=\mathbb{R}$, $C=\{0\}, A: X \rightarrow X-$ линейный, симметричный, компактный, положительно определенный оператор, $q_{0}(x)=-|x|^{2}, q_{1}(x)=\langle A x, x\rangle$. Тогда $K=\varnothing$, но в то же время $\Lambda_{0}^{\varepsilon}=\varnothing \forall \varepsilon>0$, так как индекс лежандровой квадратичной формы $\left(-q_{0} / 2+\right.$ $\left.\lambda_{1} q_{1}\right)$ конечен для любого $\lambda_{1}$ (см. $\left.[1 ; \S 6.2]\right)$ и, значит, ind $\left(q_{0}+\lambda_{1} q_{1}\right)=\infty \forall \lambda_{1}$.

ПримеР 5.2 (см. [32; пример 3]). Пусть $n=4, k=2, C=\{0\}, q_{0}(x)=$ $x_{2} x_{4}, q_{1}(x)=x_{1} x_{2}, q_{2}(x)=-x_{1}^{2}+x_{2}^{2}+x_{3}^{2}$. Непосредственно проверяется, что нерегулярные нули имеют вид $x=\left(0,0,0, x_{4}\right)$, а все остальные нетривиальные нули регулярны. Кроме того, если $Q(x)=0$, то $x_{2}=0 \Rightarrow q_{0}(x)=0$. В то же время $\omega((0,1))=-\infty$, и, значит, не выполняется ни утверждение леммы 5.1 , ни неравенство (5.7) теоремы 5.1 .

Рассмотренный пример показывает, что даже при $C=\{0\}$, если выполняется (5.1), но квадратичное отображение $Q$ имеет хотя бы один нерегулярный нетривиальный нуль $h$, для которого $q_{0}(h)=0$, то утверждение теоремы 5.1 может нарушаться. Тем не менее из теоремы 5.2 вытекает следующее следствие.

Для $\varepsilon>0$ через $\tilde{\Lambda}(\varepsilon)$ обозначим множество тех $\lambda=\left(\lambda^{0}, y^{*}\right)$, для которых $|\lambda|=1, \lambda^{0}>0, y^{*} \in C^{0}$ и существует линейное подпространство

$$
\Pi \subset X: \quad \operatorname{codim} \Pi \leqslant k-1, \quad \lambda^{0} q_{0}(x)+y^{*} Q(x)+\varepsilon \lambda^{0}|x|^{2}>0 \quad \forall x \in \Pi \backslash\{0\} .
$$

СлЕДСТвиЕ. Пустъ выполняется (5.5). Тогда для любого $x \in X \backslash\{0\}$ въполняется

$$
\forall \varepsilon>0 \quad \exists \lambda=\lambda(\varepsilon)=\left(\lambda^{0}, y^{*}\right) \in \tilde{\Lambda}(\varepsilon): \lambda^{0} q_{0}(x)+y^{*} Q(x)+\varepsilon \lambda^{0}|x|^{2}>0 .
$$

Справедливость этого утверждения вытекает из теоремы 5.2, примененной к квадратичной форме $q_{0, \varepsilon}=q_{0}(x)+\varepsilon|x|^{2}$. 
Обозначим через $\tilde{\Lambda}_{k-1}(C)=\operatorname{Ls}\{\tilde{\Lambda}(1 / i)\}$ верхний топологический предел последовательности множеств $\{\tilde{\Lambda}(1 / i)\}$. Тогда, если выполняется $(5.5)$, то

$$
\tilde{\Lambda}=\tilde{\Lambda}_{k-1}(C) \neq \varnothing, \quad \max _{\lambda \in \tilde{\Lambda}}\left(\lambda^{0} \omega(y)+\left\langle y^{*}, y\right\rangle\right) \geqslant 0 \quad \forall y
$$

Справедливость этого утверждения вытекает из семейства условий (5.16), так как любая предельная точка последовательности $\left\{\lambda_{i}\right\}: \lambda_{i} \in \tilde{\Lambda}(1 / i) \forall i$ принадлежит $\tilde{\Lambda}_{k-1}(C)$.

В свою очередь из (5.17) вытекает условие (5.3) (в котором при ненулевом конусе $C$ множество $\Lambda_{k-1}$ следует заменить на $\left.\Lambda_{k-1}(C)\right)$, поскольку, очевидно, $\tilde{\Lambda}_{k-1}(C) \subseteq \Lambda_{k-1}(C)$. Обратное, вообще говоря, неверно. Это показывает пример: $n=2, k=1, C=\{0\}, q_{0}(x)=-|x|^{2}, q_{1}(x)=x_{1}^{2}$. Для него (5.3) выполняется, а (5.17) - нет, так как, очевидно, $\tilde{\Lambda}(\varepsilon)=\varnothing \forall \varepsilon>0 \Rightarrow \tilde{\Lambda}_{k-1}(C)=\varnothing$. Таким образом, условие (5.3), необходимое для неотрицательности квадратичной формы на конусе $K$, вообще говоря, слабее условия (5.17) и, тем более, семейства условий (5.16).

Приложение к теории экстремальных задач. Вернемся к задаче (3.1), предполагая, что $X=\mathbb{R}^{n}$. Приведем условия, при которых в (2.18) можно обойтись только множителями Лагранжа, для которых $\lambda^{0}>0$.

Лемма 5.3. Пусть точка $x_{0}$ анормальна и отображение $F$ является 2-регулярным в ней, выполняется классический приниип Лагранжа (3.13) и условие квадратичного роста:

$$
\exists \delta_{1}, \delta_{2}>0: f_{0}(x)-f_{0}\left(x_{0}\right) \geqslant \delta_{1}\left|x-x_{0}\right|^{2} \quad \forall x: F(x)=0, \quad\left|x-x_{0}\right| \leqslant \delta_{2} .
$$

Тогда существует $\varepsilon>0$ такое, что

$$
\begin{gathered}
\Lambda=\left\{\lambda \in \Lambda_{k-1}\left(x_{0}\right): \lambda^{0} \geqslant \varepsilon\right\} \neq \varnothing, \\
\max _{\lambda \in \Lambda} \frac{\partial^{2} L}{\partial x^{2}}\left(x_{0}, \lambda\right)[x, x] \geqslant 0 \quad \forall x \in \operatorname{ker} F^{\prime}\left(x_{0}\right) .
\end{gathered}
$$

ДокАзАтЕльство. В силу (3.13) будем, не теряя общности, считать, что $f_{0}^{\prime}\left(x_{0}\right)=0$. По теореме 1 из [28] в силу 2-регулярности отображения $F$ в точке $x_{0}$ в ее окрестности множество $\{x: F(x)=0\}$ локально диффеоморфно конусу $H\left(x_{0}\right)$ в окрестности нуля. Отсюда, используя последнее предположение леммы, имеем $f_{0}^{\prime \prime}\left(x_{0}\right)[x, x]>0 \forall x \in H\left(x_{0}\right): x \neq 0$. Применяя теорему 5.2 , завершаем доказательство леммы.

Вернемся к достаточным условиям экстремума (4.1). Сравнивая их с необходимыми условиями (2.18), видим, что в них присутствуют различные множества множителей Лагранжа: $\Lambda\left(x_{0}\right)$ и $\Lambda_{k-1}\left(x_{0}\right)$. Это несоответствие представляется неестественным. Однако заменять в необходимых условиях множество $\Lambda_{k-1}\left(x_{0}\right)$ на большее $-\Lambda\left(x_{0}\right)$ нецелесообразно, так как это ослабление необходимых условий приводит к тому, что для анормальных задач они просто автоматически выполняются. 
В связи с этим зададимся вопросом: если заменить в (4.1) множество $\Lambda\left(x_{0}\right)$ на меньшее множество $\Lambda_{k-1}\left(x_{0}\right)$, то станут ли достаточные условия (4.1) слабее? Формально это так, но, как показывает следующая лемма, при указанной замене множеств достаточные условия (4.1) слабее не становятся.

Лемма 5.4. Пусть точка $x_{0}$ анормальна и имеет место (4.1). Тогда

$$
\max _{\lambda \in \Lambda} \frac{\partial^{2} L}{\partial x^{2}}\left(x_{0}, \lambda\right)[x, x]>0 \quad \forall x \in \operatorname{ker} F^{\prime}\left(x_{0}\right), \quad x \neq 0, \quad \Lambda=\Lambda_{k-1}\left(x_{0}\right) .
$$

Справедливость леммы вытекает из теоремы 5.2 (подробности см. в [33; c. 35,36$])$.

Сюръективность и нетривиальные нули квадратичных отображений. Зададимся вопросом: как связано наличие нетривиальных нулей у квадратичного отображения с его сюръективностью? Как отмечалось, если у квадратичного отображения $Q$ имеется регулярный нуль, то оно сюръективно. В то же время (см. [30]), все находящиеся в общем положении квадратичные отображения регулярны. Поэтому для квадратичных отображений, находящихся в общем положении, из существования нетривиального нуля вытекает сюръективность. Обратное неверно. Простейшим примером сюръективного квадратичного отображения, у которого нет нетривиальных нулей, является отображение

$$
Q: \mathbb{R}^{2} \rightarrow \mathbb{R}^{2}, \quad Q(x)=\left(x_{1} x_{2}, x_{1}^{2}-x_{2}^{2}\right) .
$$

Однако при $n \gg k$ подобных примеров уже не существует, а именно, справедливо следующее утверждение.

Лемма 5.5. Пусть квадратичное отображение $Q$ сюргективно и

$$
n>k^{2}-1 \text {. }
$$

Тогда множество его нетривиальных нулей непусто (т.е. $K \neq \varnothing)$.

Доказательство леммы, основанное на теореме 5.1, приведено в [32]. Она усиливает предложение 1 из [30], в котором доказано, что если квадратичное отображение $Q$ существенно сюръективно (существенная сюръективность означает, что любое близкое к $Q$ квадратичное отображение также сюръективно) и имеет место (5.19), то множество нетривиальных нулей у $Q$ непусто. В то же время до сих пор не ясно: существует ли сюръективное квадратичное отображение, не являющееся существенно сюръективным?

В лемме 5.5 оценку (5.19) можно улучшить. Например, для $k=2$ утверждение леммы остается в силе уже при $n \geqslant 3$. Обсудим еще вопрос: когда образ квадратичного отображения $Q(X)$ является выпуклым или почти выпуклым множеством? При этом множество $M$ называется почти выпуклым [31], если существует такое выпуклое множество $C$, что $C \subset M \subset \operatorname{cl} C$. Этот вопрос интересен как сам по себе, так и важен для приложений (подробнее см. [31] и библиографию там). Отметим только, что при $k=2$ множество $Q(X)$ выпукло [29], а уже при $k \geqslant 3$ оно может не быть даже почти выпуклым множеством. Примером служит отображение

$$
Q: \mathbb{R}^{3} \rightarrow \mathbb{R}^{3}, \quad Q(x)=\left(x_{1} x_{2}, x_{1} x_{3}, x_{2} x_{3}\right) .
$$


Для него $y_{1} y_{2} y_{3} \geqslant 0 \forall y=\left(y_{1}, y_{2}, y_{3}\right) \in Q\left(\mathbb{R}^{3}\right)$, и в то же время $Q\left(\mathbb{R}^{3}\right)$ содержит три прямые: $y_{1}=y_{2}=0, y_{2}=y_{3}=0$ и $y_{1}=y_{3}=0$.

В наиболее распространенной ситуации выпуклость $Q(X)$ гарантируется наличием регулярного нуля, так как в этом случае $Q(X)=Y$. Приведем более слабые условия, гарантирующие почти выпуклость $Q(X)$. Для целых $s \geqslant 0$ положим

$$
\mathscr{Y}_{s}=\left\{y^{*}: y^{*} \neq 0, \operatorname{ind}\left(y^{*} Q\right) \leqslant s\right\} \text {. }
$$

ЛЕмма 5.6. Пусть

$$
\exists h \in X: \quad\left\langle y^{*}, Q(h)\right\rangle<0 \quad \forall y^{*} \in \mathscr{Y}_{k-1} \backslash \mathscr{Y}_{0} .
$$

Тогда

$$
\operatorname{int}\left(\mathscr{Y}_{0}^{*}\right) \subseteq Q(X) \subseteq \mathscr{Y}_{0}^{*} .
$$

Здесь, как и выше, $\mathscr{Y}_{0}^{*}-$ сопряженный конус к конусу $\mathscr{Y}_{0}$.

СлЕДСТВИЕ. Если выполняется (5.21), то множество $Q(X)$ почти выпукло, m. е. существует такое выпуклое множество $D$, ито $D \subset Q(X) \subset \operatorname{cl} D$.

Доказательства этих утверждений приведены в [32].

Квадратичные формы, аннулирующие пересечение квадрик. Пусть заданы линейные функционалы $a_{i}, i=\overline{1, k}$. Если линейный функционал $a_{0}$ аннулирует подпространство $\left\{x:\left\langle a_{i}, x\right\rangle=0, i=\overline{1, k}\right\}$, то по лемме об аннуляторе (см. [1]) $\exists \alpha_{i}: a_{0}=\sum_{i=1}^{k} \alpha_{i} a_{i}$. Исследуем аналогичный вопрос для квадратичных отображений.

Будем говорить, что форма $q_{0}$ аннулирует конус $\{x: Q(x)=0\}$, если $q_{0}(x)=0$ $\forall x: Q(x)=0$. Спрашивается, верно ли, что тогда

$$
\exists y^{*} \in Y^{*}: Q_{0}=y^{*} Q ?
$$

Уже при $k=1$ пример, когда $Q_{1}$ - единичная матрица, показывает, что без дополнительных предположений на $Q$ ответ на этот вопрос является отрицательным (так как в этом случае конус $\{x: Q(x)=0\}=\{0\}$ аннулирует любая форма $\left.q_{0}\right)$.

Приведем условия на $Q$, при которых для любой квадратичной формы $q_{0}$, аннулирующей $\{x: Q(x)=0\}$, выполняется (5.22). (В [34] рассмотрен конус более общего вида $\{x: Q(x) \in C\}$.)

Tеорема 5.3. Пусть конус conv $\mathscr{Y}_{2(k-1)}$ является острым (т.е. не содержит ненулевых подпространств) $и$

$$
\exists h \in X: \quad\left\langle Q(h), y^{*}\right\rangle<0 \quad \forall y^{*} \in \mathscr{Y}_{k-1} .
$$

Тогда для любой квадратичной формы $q_{0}$, аннулирующей $\{x: Q(x)=0\}$, выполняется (5.22). 
ДокАЗАТЕльство. В силу доказанной ниже леммы 6.1 (см. также лемму 2 из [35]) и (5.23) отображение $Q$ сюръективно. Из остроты конуса conv $\mathscr{Y}_{2(k-1)}$ вытекает, что внутренность его поляры непуста. Поэтому

$$
\exists \hat{h} \in X: \quad\left\langle y^{*}, Q(\hat{h})\right\rangle<0 \quad \forall y^{*} \in \mathscr{Y}_{2(k-1)} .
$$

В задаче минимизации (5.2) минимум достигается в анормальной точке $x_{0}=0$. Поэтому в силу (2.18) (см. теорему 2.7) существует $\lambda=\left(\lambda^{0}, y^{*}\right) \in \Lambda_{k-1}$ такое, что $\lambda^{0} q_{0}(\hat{h})+\left\langle y^{*}, Q(\hat{h})\right\rangle \geqslant 0$. Тогда $\lambda^{0}>0$ (так как если $\lambda^{0}=0$, то $y^{*} \neq 0$, $\operatorname{ind}\left(y^{*} Q\right) \leqslant(k-1),\left\langle y^{*}, Q(\hat{h})\right\rangle \geqslant 0$, что противоречит (5.24)). Поэтому

$$
\exists y_{1}^{*}: \quad \operatorname{ind}\left(Q_{0}+y_{1}^{*} Q\right) \leqslant k-1, \quad q_{0}(\hat{h})+\left\langle y_{1}^{*}, Q(\hat{h})\right\rangle \geqslant 0 .
$$

Аналогично, заменяя в рассмотренной выше задаче (5.2) форму $q_{0}$ на $\left(-q_{0}\right)$, имеем:

$$
\exists y_{2}^{*}: \quad \operatorname{ind}\left(-Q_{0}+y_{2}^{*} Q\right) \leqslant k-1, \quad-q_{0}(\hat{h})+\left\langle y_{2}^{*}, Q(\hat{h})\right\rangle \geqslant 0 .
$$

Покажем, что $y_{2}^{*}=-y_{1}^{*}$. Предположим противное: $y_{1}^{*}+y_{2}^{*} \neq 0$. Тогда, учитывая, что индекс суммы квадратичных форм не превосходит суммы их индексов, имеем $\left(y_{1}^{*}+y_{2}^{*}\right) \in \mathscr{Y}_{2(k-1)},\left\langle\left(y_{1}^{*}+y_{2}^{*}\right), Q(\hat{h})\right\rangle \geqslant 0$. Получили противоречие с (5.24). Таким образом, $y_{2}^{*}=-y_{1}^{*}$. Поэтому, положив $y^{*}=y_{1}^{*}, \widetilde{Q}_{0}=Q_{0}+y^{*} Q$, $\tilde{q}_{0}(x)=\left\langle\widetilde{Q}_{0} x, x\right\rangle$, имеем:

$$
\text { ind } \widetilde{Q}_{0} \leqslant k-1, \quad \operatorname{ind}\left(-\widetilde{Q}_{0}\right) \leqslant k-1, \quad \tilde{q}_{0}(\hat{h})=0 .
$$

Докажем, что $\widetilde{Q}_{0}=0$. Предположим противное. Тогда $\tilde{q}_{0} \neq 0$. Поэтому в силу (5.24) существует $\tilde{h}$ достаточно близкое к $\hat{h}$, для которого

$$
\left\langle\mu^{*}, Q(\tilde{h})\right\rangle<0 \quad \forall \mu^{*} \in \mathscr{Y}_{2(k-1)}, \quad \tilde{q}_{0}(\tilde{h}) \neq 0 .
$$

Построенная квадратичная форма $\tilde{q}_{0}$ также аннулирует $\{x: Q(x)=0\}$. Поэтому, проводя для нее и для вектора $\tilde{h}$ рассуждения, аналогичные приведенным выше, имеем

$$
\begin{gathered}
\exists \tilde{y}^{*}: \quad \operatorname{ind}\left(\widetilde{Q}_{0}+\widetilde{y}^{*} Q\right) \leqslant k-1, \quad \operatorname{ind}\left(-\left(\widetilde{Q}_{0}+\tilde{y}^{*} Q\right)\right) \leqslant k-1, \\
\tilde{q}_{0}(\tilde{h})+\left\langle\tilde{y}^{*}, Q(\tilde{h})\right\rangle=0 .
\end{gathered}
$$

Из первого неравенства (5.25) и второго неравенства (5.27) имеем

$$
\begin{gathered}
2(k-1) \geqslant \operatorname{ind}\left(\widetilde{Q}_{0}-\left(\widetilde{Q}_{0}+\tilde{y}^{*} Q\right)\right)=\operatorname{ind}\left(-\tilde{y}^{*} Q\right) \\
\Rightarrow \operatorname{ind}\left(-\tilde{y}^{*} Q\right) \leqslant 2(k-1) .
\end{gathered}
$$

Аналогично из второго неравенства (5.25) и первого неравенства (5.27) получаем $\operatorname{ind}\left(\tilde{y}^{*} Q\right) \leqslant 2(k-1)$. Таким образом, $\pm \tilde{y}^{*} \in \mathscr{Y}_{2(k-1)} \Rightarrow \tilde{y}^{*}=0$, так как конус $\operatorname{conv} \mathscr{Y}_{2(k-1)}$ острый. Но из равенства в $(5.27)$, в силу $\tilde{y}^{*}=0$, имеем $\tilde{q}_{0}(\tilde{h})=0$. Полученное противоречие с (5.26) доказывает, что $\widetilde{Q}_{0}=0 \Rightarrow Q_{0}+y^{*} Q=0$. Теорема доказана. 
Теория квадратичных отображений находится только в самом начале своего развития. Поэтому мы приведем несколько пока нерешенных задач этой теории.

ЗАДАча 1. Пусть заданное квадратичное отображение $Q: \mathbb{R}^{n} \rightarrow \mathbb{R}^{k}$ сюръективно. Спрашивается, будет ли любое достаточно близкое к нему квадратичное отображение также сюръективно? (Близость квадратичных отображений понимается в том очевидном смысле, что определяющие их матрицы попарно близки.) При дополнительном предположении, что конус conv $\mathscr{Y}_{k}$ является острым, положительный ответ на этот вопрос дан в [35]. В общем случае ответ пока не известен.

ЗАДАчА 2. Пусть квадратичное отображение $Q: \mathbb{R}^{n} \rightarrow \mathbb{R}^{k}$ сюръективно. Спрашивается, верно ли, что $0 \in \operatorname{int} Q(B)$ ? Здесь $B$ - единичный шар в $\mathbb{R}^{n}$.

ЗАДАчА 3. Пусть у заданного квадратичного отображения $Q: \mathbb{R}^{n} \rightarrow \mathbb{R}^{k}$ нет нетривиальных нулей. Тогда для него множество $\mathscr{Y}_{k-1}$ непусто. Это вытекает из теоремы 2.7, примененной к задаче (5.2) при $q_{0}(x) \equiv-|x|^{2}$.

При $k=2,3,5,9$ в [36] приведены примеры квадратичных отображений, у которых нет нетривиальных нулей, для которых, однако, $\mathscr{Y}_{k-2}=\varnothing$. Спрашивается, существуют ли примеры таких квадратичных отображений при $k \neq$ $2,3,5,9$ ?

\section{6. Теоремы об обратной функции}

Индексный подход. Пусть задано отображение $F: X \rightarrow Y$, где $X$ - линейное пространство и $Y=\mathbb{R}^{k}$. Дана точка $x_{0} \in X$ и $y_{0}=F\left(x_{0}\right)$. Приведем условия, при которых существует такая окрестность $V$ точки $y_{0}$, что для любого $y \in V$ уравнение (1.6) имеет такое решение $x(y)$, что $x\left(y_{0}\right)=x_{0}$, отображение $x(\cdot)$ непрерывно в точке $y_{0}$ относительно конечной топологии и удовлетворяет априорным оценкам.

Теорема 6.1. Предположим, что отображение $F$ дважды непрерывно дифферениируемо в окрестности точки $x_{0}$ относителъно конечной топологии $\tau$. Пусть

$$
\exists h \in X: \quad F^{\prime}\left(x_{0}\right) h=0, \quad\left\langle y^{*}, F^{\prime \prime}\left(x_{0}\right)[h, h]\right\rangle<0 \quad \forall y^{*} \in \mathscr{F}_{k}^{2}\left(x_{0}\right) .
$$

Тогда существуют такие константа const, окрестность $V$ точки у и и линейное конечномерное подпространство $\widetilde{\Pi} \subseteq X$, что

$$
\begin{gathered}
\forall y \in V \quad \exists x(y) \in \widetilde{\Pi}: F(x(y))=y, \\
\left\|x(y)-x_{0}\right\|_{\widetilde{\Pi}} \leqslant \operatorname{const}\left(\left|y-y_{0}\right|+\left|\pi\left(y-y_{0}\right)\right|^{1 / 2}\right) .
\end{gathered}
$$

Здесь, как и выше, $\pi$ - оператор ортогонального проектирования $Y$ на $\left(\operatorname{im} F^{\prime}\left(x_{0}\right)\right)^{\perp}$, a $\mathscr{F}_{s}^{2}(x)$ - множество таких $y^{*} \in Y^{*}$, что $\left|y^{*}\right|=1, F^{\prime}(x)^{*} y^{*}=0$ и в $X$ существует подпространство П, для которого выполняется (2.19).

Кроме того, если $0 \notin$ conv $\mathscr{F}_{k}^{2}\left(x_{0}\right)$ и выполняется (6.2), то имеет место (6.1). Но, как отмечалось в разделе 2 (см. также [5; гл. $1, \S 1.9])$, при $n \gg k$ для 
отображений общего положения $0 \notin \operatorname{conv} \mathscr{F}_{k}^{2}(x) \forall x$ и, значит, для них теорема 6.1 дает не только достаточные, но и необходимые условия существования обратной функции $x(\cdot)$.

Если точка $x_{0}$ нормальна, то $\mathscr{F}_{k}^{2}\left(x_{0}\right)=\varnothing, \pi=0$ и теорема 6.1 по существу превращается в классическую теорему об обратной функции (при этом возможность выбора гладкого отображения $x(\cdot)$ доказывается отдельно и несложно). Если же точка $x_{0}$ анормальна, то $\pi \neq 0$ и оценка на решение (6.2) является всего лишь “линейно-корневой”, в отличие от линейной оценки, как в классическом случае.

Доказательство приведенных утверждений в общем случае, основанное на необходимых условиях второго порядка из раздела 2, приведено в [35]. Чтобы продемонстрировать основную идею доказательства теоремы, докажем следующую лемму, из которой теорема 6.1 вытекает в частном случае, когда $x_{0}=0$, а $F$ - квадратичное отображение.

Лемма 6.1. Пусть задано квадратичное отображение $Q: \mathbb{R}^{n} \rightarrow Y=\mathbb{R}^{k}$, $k \geqslant 2, u$

$$
\exists h \in \mathbb{R}^{n}: \quad\left\langle Q(h), y^{*}\right\rangle<0 \quad \forall y^{*} \in \mathscr{Y}_{k-2}
$$

(см. (5.20)). Тогда существует константа const такая, что

$$
\forall y \in Y \quad \exists x(y) \in \mathbb{R}^{n}: \quad Q(x(y))=y, \quad|x(y)| \leqslant \text { const }|y|^{1 / 2} .
$$

ДокАзАТЕльство. Предположим противное. Тогда, как несложно видеть, для любого $i$ существует $y_{i} \in Y$ такое, что $\left|y_{i}\right|=1$ и в задаче минимизации

$$
|x|^{2}-i \chi \rightarrow \min , \quad Q(x)-\chi y_{i}=0, \quad(x, \chi) \in \mathbb{R}^{n} \times \mathbb{R}
$$

точка $x=0, \chi=0$ является решением. В силу необходимых условий второго порядка (2.18) для этой задачи существуют $\lambda_{i}^{0} \geqslant 0, y_{i}^{*} \in Y: \lambda_{i}^{0}+\left|y_{i}^{*}\right|=1$ и линейное подпространство $\widetilde{\Pi}_{i} \subseteq \mathbb{R}^{n} \times \mathbb{R}$ такие, что

$$
\begin{gathered}
\operatorname{codim} \widetilde{\Pi}_{i} \leqslant k-1, \quad \chi=0 \quad \forall(x, \chi) \in \widetilde{\Pi}_{i}, \quad i \lambda_{i}^{0}+\left\langle y_{i}^{*}, y_{i}\right\rangle=0, \\
\left\langle y_{i}^{*}, Q(x)\right\rangle+\lambda_{i}^{0}|x|^{2} \geqslant 0 \quad \forall x \in \Pi_{i}=\left\{x \in \mathbb{R}^{n}:(x, \chi) \in \widetilde{\Pi}_{i}\right\}, \\
\left\langle y_{i}^{*}, Q(h)\right\rangle+\lambda_{i}^{0}|h|^{2} \geqslant 0 .
\end{gathered}
$$

Тогда $\operatorname{codim} \Pi_{i} \leqslant k-2 \Rightarrow \operatorname{ind}\left(y_{i}^{*} Q+\lambda_{i}^{0} I\right) \leqslant k-2$ ( $I$ - единичная матрица). Кроме того, $\lambda_{i}^{0} \rightarrow 0 \Rightarrow\left|y_{i}^{*}\right| \rightarrow 1$. Переходя к подпоследовательности, будем считать, что $y_{i}^{*} \rightarrow y^{*},\left|y^{*}\right|=1$. Переходя к пределу при $i \rightarrow \infty$, имеем $\left\langle y^{*}, Q(h)\right\rangle \geqslant 0$, и в силу теоремы $2.3 y^{*} \in \mathscr{Y}_{k-2}$. Получили противоречие с (6.3). Лемма доказана.

При $k=1$ (6.3) надо заменить на (5.23) или на $\mathscr{Y}_{0}=\varnothing$, что при $k=1$ эквивалентно.

Лемма 6.1 дает достаточные условия сюръективности квадратичных отображений. Важно отметить, что с учетом примера 4 из [37] пример отображения (5.18) показывает, что в утверждении леммы 6.1 непрерывного отображения $x(\cdot)$ может не быть. 
2-регулярность. В приложениях нередко бывает недостаточно просто решить уравнение (1.6), а нужно найти такие его решения $x$, что $x \in K$, где $K-$ заданное подмножество $Y$. Естественно, наряду с анормальностью, ограничение $x \in K$ привносит в эту задачу дополнительные сложности.

Будем рассматривать следующую, более общую, чем (1.6), задачу нахождения решения уравнения

$$
F(x, \sigma)=0, \quad x \in K
$$

Здесь $X, Y$ - банаховы пространства, $\Sigma$ - топологическое пространство, $K \subseteq$ $X$ - выпуклый замкнутый конус, а $F: X \times \Sigma \rightarrow Y$ - заданное отображение. Пусть имеются $x_{0} \in K, \sigma_{0} \in \Sigma$, для которых $F\left(x_{0}, \sigma_{0}\right)=0$.

В предположении гладкости $F$ из теоремы об устойчивости Робинсона [16], [14] вытекает, что если выполнено условие регулярности Робинсона

$$
\frac{\partial F}{\partial x}\left(x_{0}, \sigma_{0}\right)\left(K+\operatorname{span}\left\{x_{0}\right\}\right)=Y
$$

(при $K=X$ условие Робинсона - это условие нормальности), то существует такая окрестность $O$ точки $\sigma_{0}$, что $\forall \sigma \in O \exists x(\sigma) \in K$ :

$$
F(x(\sigma), \sigma) \equiv 0, \quad\left\|x(\sigma)-x_{0}\right\| \leqslant \mathrm{const}\left\|F\left(x_{0}, \sigma\right)\right\| \quad \forall \sigma \in O .
$$

Правда, вопрос о возможности выбора отображения $x(\cdot)$ непрерывным в цитированных работах не обсуждался. Тем не менее справедлива следующая теорема.

ТЕоремА 6.2 (классическая теорема о неявной функции). Предположим, что отображение $F: X \times \Sigma \rightarrow Y$ непрерывно в окрестности точки $\left(x_{0}, \sigma_{0}\right)$, строго дифферениируемо по $x$ в этой точке равномерно по $\sigma$, а также что въполнено условие Робинсона (6.5). Тогда существуют окрестность О точки $\sigma_{0}$ u непрерывное отображсение $x(\cdot): O \rightarrow K$ такие, что имеет место (6.6).

Перейдем к теоремам о неявной функции без априорных предположений условия Робинсона. Относительно $F$ будем предполагать, что в окрестности точки $\left(x_{0}, \sigma_{0}\right)$ оно дважды непрерывно дифференцируемо по $x$ равномерно по $\sigma$ и при каждом фиксированном $\sigma$ его вторая частная производная $\frac{\partial^{2} F}{\partial x^{2}}(\cdot, \sigma)$, рассматриваемая как симметричное билинейное отображение, удовлетворяет условию Липшица по переменной $x$ с одной и той же константой Липшица, не зависящей от $\sigma$. (Подробнее см. [38].) Отображения $F\left(x_{0}, \cdot\right), \frac{\partial F}{\partial x}\left(x_{0}, \cdot\right), \frac{\partial^{2} F}{\partial x^{2}}\left(x_{0}, \cdot\right)$ предполагаются непрерывными в окрестности точки $\sigma_{0}$.

Положим

$$
\mathscr{K}=K+\operatorname{span}\left\{x_{0}\right\}, \quad C=\frac{\partial F}{\partial x}\left(x_{0}, \sigma_{0}\right)(\mathscr{K}) .
$$

Будем предполагать, что линейная оболочка $\operatorname{span} C$ конуса $C$ замкнута и это подпространство топологически дополняемо. Через $\pi$ обозначим линейный непрерывный оператор, проектирующий $Y$ на какое-нибудь подпространство, дополняющее $\operatorname{span} C$. 
ОПРЕДЕЛЕНИЕ. Пусть

$$
h \in \mathscr{K}, \quad \frac{\partial F}{\partial x}\left(x_{0}, \sigma_{0}\right) h=0, \quad-\frac{\partial^{2} F}{\partial x^{2}}\left(x_{0}, \sigma_{0}\right)[h, h] \in C .
$$

Тогда отображение $F$ называется 2-регулярным в точке $\left(x_{0}, \sigma_{0}\right)$ относительно $K$ по направлению $h$, если имеет место равенство

$$
\frac{\partial F}{\partial x}\left(x_{0}, \sigma_{0}\right)(\mathscr{K})+\frac{\partial^{2} F}{\partial x^{2}}\left(x_{0}, \sigma_{0}\right)\left[h, \mathscr{K} \cap \operatorname{ker} \frac{\partial F}{\partial x}\left(x_{0}, \sigma_{0}\right)\right]=Y .
$$

В предположении ri $C \neq \varnothing$ другое, но эквивалентное введенному определение 2-регулярности приведено в [39], и оба они при $K=X$ превращаются в условие 2-регулярности, рассматривавшееся в предыдущих разделах.

Теорема 6.3. Пусть ri $C \neq \varnothing$ и существует такое $h \in X$, что отображение $F$ является 2-регулярным в точке $\left(x_{0}, \sigma_{0}\right)$ относительно $K$ по направлению $h$ (т.е. выполняется (6.7), (6.8)). Тогда для любого $l \in \operatorname{ri} C$ существуют окрестность $O$ точки $\sigma_{0}, \delta>0$ и непрерывное отображение $x(\cdot): O \rightarrow K$ такие, что

$$
\begin{gathered}
F(x(\sigma), \sigma)=0 \quad \forall \sigma \in O \\
\left\|x(\sigma)-x_{0}\right\| \leqslant \operatorname{const}\left(\Delta_{1}(\sigma)+\Delta_{2}(\sigma)+\left\|F\left(x_{0}, \sigma\right)\right\|\right. \\
\left.+\rho\left(-F\left(x_{0}, \sigma\right), C_{\delta}\right)^{1 / 2}\right) \quad \forall \sigma \in O .
\end{gathered}
$$

Здесь

$$
\begin{aligned}
& \Delta_{1}(\sigma)=\sup \left\{\left\|\pi \frac{\partial F}{\partial x}\left(x_{0}, \sigma\right) x\right\|, x \in \operatorname{span} K,\|x\| \leqslant 1\right\}, \\
& \Delta_{2}(\sigma)=\sup \left\{\left\|\frac{\partial F}{\partial x}\left(x_{0}, \sigma\right) x\right\|, x \in \operatorname{ker} \frac{\partial F}{\partial x}\left(x_{0}, \sigma_{0}\right) \cap \mathscr{K},\|x\| \leqslant 1\right\},
\end{aligned}
$$

$C_{\delta}=\operatorname{cone}\left(B_{\delta}(l)\right) \cap \operatorname{span} C$, а сопе обозначает коническую оболочку множества.

При дополнительном предположении:

$$
\text { конус } \frac{\partial F}{\partial x}\left(x_{0}, \sigma_{0}\right)(K) \text { является подпространством }
$$

оценку (6.10) в теореме 6.3 можно усилить, опустив в ней слагаемое $\Delta_{2}(\sigma)$. А именно, справедлива следующая теорема.

ТЕОРема 6.4. Пусть выполнены все предположения теоремы 6.3, а также условие (6.11). Тогда существуют окрестность $О$ точки $\sigma_{0}$ и непрерывное отображение $x(\cdot): O \rightarrow K$ такие, что выполняются (6.9) и оценка

$$
\left\|x(\sigma)-x_{0}\right\| \leqslant \operatorname{const}\left(\Delta_{1}(\sigma)+\left\|F\left(x_{0}, \sigma\right)\right\|+\left\|\pi F\left(x_{0}, \sigma\right)\right\|^{1 / 2}\right) \quad \forall \sigma \in O .
$$

Классическая теорема о неявной функции является следствием теоремы 6.4 (так как если выполняется условие Робинсона, то $\pi=0$ ). Из теоремы 6.4 
получается также обобщенная теорема о неявной функции, обусловленная решением уравнения $F(x, \sigma)=y$ относительно неизвестного $x \in K$ (подробнее см. теорему 5 из [38]).

Из теоремы 6.4 и результатов работы [24] можно вывести теорему 6.1, даже усилив ее в анормальном случае. А именно, пусть точка $x_{0}$ анормальна и

$$
\exists h \in X: \quad F^{\prime}\left(x_{0}\right) h=0, \quad\left\langle y^{*}, F^{\prime \prime}\left(x_{0}\right)[h, h]\right\rangle<0 \quad \forall y^{*} \in \mathscr{F}_{k-1}^{2}\left(x_{0}\right) .
$$

Тогда существуют окрестность $V$ точки $y_{0}$, линейное конечномерное подпространство $\widetilde{\Pi} \subseteq X$ и непрерывное отображение $x(\cdot): V \rightarrow \widetilde{\Pi}$, для которых имеет место (6.2).

Действительно, в силу (6.13) и [23; теорема 2] существует направление $h \in X$, вдоль которого отображение $F$ является 2-регулярным в точке $x_{0}$. Поэтому искомое утверждение вытекает из теоремы 6.4 при $K=X$, так как в предположениях теоремы 6.1 отображение $F$ линейно по переменной $\sigma$ и, значит, при $K=X$ в (6.12) слагаемое $\Delta_{1}$ равно нулю.

Теоремы 6.3 и 6.4 справедливы лишь в предположении непустоты конуса ri $C$. Однако, если пространство $Y$ бесконечномерно, то относительная внутренность лежащего в нем выпуклого конуса $C$ может быть пуста. Поэтому приведем теорему о неявной функции, справедливую без априорного предположения непустоты ri $C$.

Теорема 6.5. Пусть отображение $F$ является 2-регулярным в точке $\left(x_{0}, \sigma_{0}\right)$ относительно $K$ по некоторому направлению $h \in X$ (т.е. имеет место (6.7), (6.8)). Тогда существует окрестность $O$ точки $\sigma_{0}$ и непрерывное отображение $x(\cdot): O \rightarrow K$ такие, что выполняются (6.9) и оценка

$$
\left\|x(\sigma)-x_{0}\right\| \leqslant \operatorname{const}\left(\Delta_{1}(\sigma)+\Delta_{2}(\sigma)+\left\|F\left(x_{0}, \sigma\right)\right\|^{1 / 2}\right) \quad \forall \sigma \in O .
$$

Эти теоремы получены в [38], [40] и там же приведены иллюстрирующие их примеры. Отметим, что в этих теоремах оценки на $\left\|x(\sigma)-x_{0}\right\|$ лишь линейно-корневые, а не линейные, как в классической теореме о неявной функции.

Теорема о неявной функции в анормальной точке при $K=X$ получена в [41], где в предположении 2-регулярности $F$ по некоторому направлению доказано существование отображения $x(\cdot)$, удовлетворяющего (6.9) и оценке $(6.12)$ при $\Delta_{1}=0$. Вопрос непрерывности отображения $x(\cdot)$ в [41] не рассматривался.

Уравнения с незамкнутым образом. Вернемся к уравнению (1.6), предполагая, что $X$ и $Y$ - банаховы пространства. Теоремы 6.3-6.5 применимы и к уравнению (1.6), но в предположении, что образ $\operatorname{im} F^{\prime}\left(x_{0}\right)$ замкнут. Однако, если точка $x_{0}$ анормальна, а пространство $Y$ бесконечномерно, то это предположение может оказаться обременительным. Приведем теорему об обратной функции, в которой предположение замкнутости im $F^{\prime}\left(x_{0}\right)$ отсутствует. Будем считать, что отображение $F$ дважды непрерывно дифференцируемо и его вторая производная липшицева в окрестности $x_{0}$. 
Теорема 6.6. Пусть отображение F является 2-регулярным по некоторому направлению $h$, удовлетворяющему условиям (3.15). Тогда найдутся такие $r, c, c_{1}>0$, что для любого $l \in \operatorname{im} F^{\prime}\left(x_{0}\right)$ и любого $y \in B_{Y}\left(y_{0}, r(l)\right)$ существует $x(y) \in B_{X}\left(x_{0}, r\right)$ maкое, что

$F(x(y))=y, \quad\left\|x(y)-x_{0}\right\| \leqslant c_{1}\left(b(l)\left\|y-y_{0}\right\|+\left\|y-y_{0}\right\|^{1 / 2}\left\|\theta\left(y-y_{0}\right)-\theta(l)\right\|^{1 / 2}\right)$.

Здесь $y_{0}=F\left(x_{0}\right), B_{X}(x, \rho)$ - шар в $X$ радиуса $\rho$ с центром в точке $x$ и использованы обозначения: $\theta(y)=y /\|y\|$ при $y \neq 0$ и $\theta(0)=0, b(y)=$ $\inf \left\{\|x\|: F^{\prime}\left(x_{0}\right) x=\theta(y)\right\}$ для $y \in \operatorname{im} F^{\prime}\left(x_{0}\right), r(l)=\min \left\{c r / b(l), c r^{2}\right\}$. Из этой теоремы при $l=0$ вытекает важное следствие.

СледствиЕ. B предположениях теоремы 6.6 существуют такие $r, c>0$, чmo

$$
\forall y \in B_{Y}\left(y_{0}, r\right) \quad \exists x(y): \quad F(x(y))=y, \quad\left\|x(y)-x_{0}\right\| \leqslant c\left\|y-y_{0}\right\|^{1 / 2} .
$$

Теорема 6.7. Пусть отображение F является 2-регулярным по некоторому направлению $h \in H\left(x_{0}\right)(c м .(3.2))$. Тогда существуют такие константы $r, c, c_{1}, c_{2}>0$, что для любого $l \in \operatorname{im} F^{\prime}\left(x_{0}\right)$ и любого $y \in B_{Y}\left(y_{0}, r(l)\right)$ выполняется

$$
\exists x(y) \in B_{X}\left(x_{0}, r\right): F(x(y))=y, \quad x(y)=x_{0}+t(y)\left(h+\xi+\chi_{1}\right)+O\left(t^{2}(y)\right) .
$$

Здесь $r(l)$ определено выше и

$$
t(y)=\max \left\{c_{1}\left\|y-y_{0}\right\| b(l) r^{-1}, c_{2}\left\|y-y_{0}\right\|^{1 / 2}\left\|\theta\left(y-y_{0}\right)-\theta(l)\right\|^{1 / 2} r^{-1 / 2}\right\},
$$

a $\xi, \chi_{1}$ - произвольные решения некоторой линейно-квадратичной системь уравнений (ее явный вид приведен в теореме 2 из [22]).

Теорема включает в себя утверждение о существовании указанных $\xi$ и $\chi_{1}$. Доказательства обеих теорем и иллюстрирующие их примеры приведены в [22].

\section{7. Необходимые условия второго порядка в задачах оптимального управления}

Задача Лагранжа. В качестве одного из приложений результатов раздела 2 получим для задач оптимального управления необходимые условия слабого минимума. Вначале рассмотрим задачу Лагранжа, т. е. задачу без ограничений на управления:

$$
\begin{gathered}
\dot{x}=f(x, u, t), \quad t \in\left[t_{1}, t_{2}\right] \\
K_{1}(p) \leqslant 0, \quad K_{2}(p)=0, \quad \text { где } \quad p=\left(x_{1}, x_{2}\right), \quad x_{1}=x\left(t_{1}\right), \quad x_{2}=x\left(t_{2}\right), \\
J=J(p, u)=K_{0}(p)+\int_{t_{1}}^{t_{2}} f^{0}(x, u, t) d t \rightarrow \min .
\end{gathered}
$$

Здесь $t \in\left[t_{1}, t_{2}\right]$ - время, причем моменты $t_{1}<t_{2}$ фиксированы, $x \in \mathbb{R}^{n}$ - фазовая переменная, а $u \in \mathbb{R}^{m}$ - управляющий параметр; $f$ - заданная $n$-мерная 
вектор-функция, а $K_{0}$ и $f^{0}$ - скалярные функции. Вектор-функции $K_{1}$ и $K_{2}$ принимают значения в арифметических пространствах размерностей $d\left(K_{1}\right)$ и $d\left(K_{2}\right)$ соответственно. Здесь и ниже $d(z)$ - размерность вектора $z$.

Относительно функций $K_{0}, K_{1}$ и $K_{2}$ предполагается, что они дважды непрерывно дифференцируемы. Функции $f^{0}, f$ предполагаются дважды непрерывно дифференцируемыми по $(x, u)$ для почти всех $t$. Кроме того, эти функции вместе со всеми своими частными производными по $(x, u)$ до второго порядка включительно измеримы по $t$ при любых фиксированных $(x, u)$, и на любом ограниченном множестве они ограничены и непрерывны по $(x, u)$ равномерно по $x, u, t$. В качестве класса допустимых управлений рассматривается пространство функций $u=u(\cdot) \in L_{\infty}^{m}\left[t_{1}, t_{2}\right]$.

Относительно ограничений (7.2), называемых концевыми, предполагается выполненным следующее условие регулярности: для любого $p$, удовлетворяющего (7.2), имеет место

$$
\begin{gathered}
\operatorname{rank} \frac{\partial K_{2}}{\partial p}(p)=d\left(K_{2}\right) ; \\
\exists \tilde{p}=\left(\tilde{x}_{1}, \tilde{x}_{2}\right) \in \mathbb{R}^{2 n}: \tilde{p} \frac{\partial K_{2}}{\partial p}(p)=0, \quad\left\langle\frac{\partial K_{1, j}}{\partial p}(p), \tilde{p}\right\rangle<0 \\
\forall j: K_{1, j}(p)=0, \quad j=\frac{1, d\left(K_{1}\right) .}{}
\end{gathered}
$$

Пара вектор-функций $(x(t), u(t)), t \in\left[t_{1}, t_{2}\right]$, называется допустимым процессом, если $u(\cdot)$ - допустимое управление, а $x(\cdot)$ - соответствующее ему решение уравнения (7.1), которое удовлетворяет концевым ограничениям (7.2). Исходная задача заключается в нахождении минимума функционала $J$ на множестве допустимых процессов.

Положим $K=\left(K_{1}, K_{2}\right)$. Определим функцию Понтрягина $H: \mathbb{R}^{n} \times \mathbb{R}^{m} \times$ $\mathbb{R} \times\left(\mathbb{R}^{n}\right)^{*} \times \mathbb{R} \rightarrow \mathbb{R}$ и малый лагранжиан $l: \mathbb{R}^{2 n} \times \mathbb{R}^{1+d(K)} \rightarrow \mathbb{R}$ по формулам

$$
\begin{gathered}
H\left(x, u, t, \psi, \lambda^{0}\right)=\langle f(x, u, t), \psi\rangle-\lambda^{0} f^{0}(x, u, t), \\
l(p, \lambda)=\lambda^{0} K_{0}(p)+\left\langle\lambda^{1}, K_{1}(p)\right\rangle+\left\langle\lambda^{2}, K_{2}(p)\right\rangle ; \quad \lambda=\left(\lambda^{0}, \lambda^{1}, \lambda^{2}\right) \in \mathbb{R}^{1+d(K)} .
\end{gathered}
$$

Здесь $\lambda^{0} \in \mathbb{R}, \lambda^{s} \in \mathbb{R}^{d\left(K_{s}\right)}, s=1,2$, а $\psi$ - это $n$-мерный вектор-столбец.

Будем говорить, что допустимый процесс $\left(x_{0}(\cdot), u_{0}(\cdot)\right)$ удовлетворяет уравнению Эйлера-Лагранжа, если существует такой вектор $\lambda$, удовлетворяющий условиям

$$
|\lambda|=1, \quad \lambda^{0} \geqslant 0, \quad \lambda^{1} \geqslant 0, \quad\left\langle\lambda^{1}, K_{1}\left(p_{0}\right)\right\rangle=0,
$$

что для вектор-функции $\psi$, являющейся решением задачи Коши

$$
\dot{\psi}=-\frac{\partial H}{\partial x}\left(x_{0}(t), u_{0}(t), t, \psi, \lambda^{0}\right), \quad \psi\left(t_{1}\right)=\frac{\partial l}{\partial x_{1}}\left(p_{0}, \lambda\right),
$$

имеет место

$$
\begin{gathered}
\psi\left(t_{2}\right)=-\frac{\partial l}{\partial x_{2}}\left(p_{0}, \lambda\right), \quad \text { где } \quad p_{0}=\left(x_{0}\left(t_{1}\right), x_{0}\left(t_{2}\right)\right), \\
\frac{\partial H}{\partial u}\left(x_{0}(t), u_{0}(t), t, \psi(t), \lambda^{0}\right)=0 \quad \dot{\forall} t .
\end{gathered}
$$


Здесь $\dot{\forall} t$ означает “для почти всех $t$ ”. Отметим, что в силу регулярности концевых ограничений $\lambda^{0}+|\psi(t)|>0 \forall t$.

Обозначим через $\Lambda\left(x_{0}(\cdot), u_{0}(\cdot)\right)$ множество векторов $\lambda$, отвечающих допустимому процессу $\left(x_{0}(\cdot), u_{0}(\cdot)\right)$ в силу уравнения Эйлера-Лагранжа, дающее необходимые условия экстремума первого порядка, вообще говоря, более слабые, чем принцип максимума Понтрягина и эквивалентные ему для задач, линейных по управлению. Процесс, удовлетворяющий уравнению Эйлера-Лагранжа, называется экстремалью.

Будем рассматривать экстремаль $\left(x_{0}(\cdot), u_{0}(\cdot)\right)$. В дальнейшем, снова для удобства удаляя из концевых ограничений (7.2) все ограничения типа неравенств, отвечающие неактивным индексам, будем считать, что $K_{1}\left(p_{0}\right)=0$. Система уравнений в вариациях, отвечающая этой экстремали, имеет вид

$$
\begin{gathered}
\frac{d}{d t} \delta x=\delta x \frac{\partial f}{\partial x}\left(x_{0}(t), u_{0}(t), t\right)+\delta u(t) \frac{\partial f}{\partial u}\left(x_{0}(t), u_{0}(t), t\right), \\
\delta u \in L_{\infty}^{m}\left[t_{1}, t_{2}\right] .
\end{gathered}
$$

Рассмотрим пространство $X=\mathbb{R}^{n} \times L_{\infty}^{m}\left[t_{1}, t_{2}\right]$, а также лежащий в нем конус $\mathscr{K}\left(x_{0}(\cdot), u_{0}(\cdot)\right)$ - конус критических направлений, состоящий из таких пар $(\xi, \delta u)$, что

$$
\left(\delta x\left(t_{1}\right), \delta x\left(t_{2}\right)\right) \frac{\partial K_{1}}{\partial p}\left(p_{0}\right) \leqslant 0, \quad\left(\delta x\left(t_{1}\right), \delta x\left(t_{2}\right)\right) \frac{\partial K_{2}}{\partial p}\left(p_{0}\right)=0 .
$$

Здесь и ниже $\delta x$ - соответствующее $\delta u$ решение системы уравнений в вариациях $(7.8)$ с начальным условием $\delta x\left(t_{1}\right)=\xi$. Положим

$$
\mathscr{N}_{K}=\left\{(\xi, \delta u) \in X:\left(\delta x\left(t_{1}\right), \delta x\left(t_{2}\right)\right) \frac{\partial K}{\partial p}\left(p_{0}\right)=0\right\} .
$$

Очевидно, что $\mathscr{N}_{K}$ - это максимальное линейное подпространство, содержащееся в $\mathscr{K}\left(x_{0}(\cdot), u_{0}(\cdot)\right)$.

Для $\lambda \in \Lambda\left(x_{0}(\cdot), u_{0}(\cdot)\right)$ на пространстве $X$ определим квадратичную формy $\Gamma(\lambda)$ :

$$
\begin{aligned}
\Gamma(\xi, \delta u ; \lambda) & =\frac{\partial^{2} l}{\partial p^{2}}\left(p_{0}, \lambda\right)\left[\left(\delta x\left(t_{1}\right), \delta x\left(t_{2}\right)\right),\left(\delta x\left(t_{1}\right), \delta x\left(t_{2}\right)\right)\right] \\
& -\int_{t_{1}}^{t_{2}} \frac{\partial^{2} H}{\partial(x, u)^{2}}\left(x_{0}(t), u_{0}(t), t, \psi(t), \lambda^{0}\right)[(\delta x(t), \delta u(t)),(\delta x(t), \delta u(t))] d t .
\end{aligned}
$$

Пусть $\Phi$ - фундаментальная матрица системы уравнений в вариациях (7.8), т. е. $\Phi-$ решение линейной однородной системы $\frac{d}{d t} \Phi=\Phi \frac{\partial f}{\partial x}\left(x_{0}(t), u_{0}(t), t\right)$, $\Phi\left(t_{1}\right)=I$, где $I-$ единичная матрица. Положим

$$
A=\frac{\partial K}{\partial x_{1}}\left(p_{0}\right)+\Phi\left(t_{2}\right) \frac{\partial K}{\partial x_{2}}\left(p_{0}\right), \quad B(t)=\frac{\partial f}{\partial u}\left(x_{0}(t), u_{0}(t), t\right) \Phi^{-1}(t) \Phi\left(t_{2}\right) \frac{\partial K}{\partial x_{2}}\left(p_{0}\right)
$$

и введем в рассмотрение расширенную матрицу управляемости

$$
D=A^{*} A+\int_{t_{1}}^{t_{2}} B^{*}(t) B(t) d t
$$


(размера $d(K) \times d(K))$. Через $d$ обозначим размерность ядра матрицы $D$. Наконец через $\Lambda_{\theta}=\Lambda_{\theta}\left(x_{0}(\cdot), u_{0}(\cdot)\right)$ обозначим множество тех $\lambda \in \Lambda\left(x_{0}(\cdot), u_{0}(\cdot)\right)$, для которых индекс сужения формы $\Gamma(\lambda)$ на подпространство $\mathscr{N}_{K}$ не превышает числа $\theta=\min (d, 2 n)$.

Теорема 7.1. Пусть допустимый процесс $\left(x_{0}(\cdot), u_{0}(\cdot)\right)$ является слабым минимумом в задаче (7.1)-(7.3). Тогда $\Lambda_{\theta} \neq \varnothing$ и для произволъных $(\xi, \delta u) \in \mathscr{K}$, удовлетворяющих

$$
\left\langle\left(\delta x\left(t_{1}\right), \delta x\left(t_{2}\right)\right), \frac{\partial K_{0}}{\partial p}\left(p_{0}\right)\right\rangle+\int_{t_{1}}^{t_{2}}\left\langle(\delta x(t), \delta u(t)), \frac{\partial f^{0}}{\partial(x, u)}\left(x_{0}(t), u_{0}(t), t\right)\right\rangle d t \leqslant 0,
$$

имеет место неравенство

$$
\max _{\lambda \in \Lambda_{\theta}} \Gamma(\xi, \delta u ; \lambda) \geqslant 0
$$

Доказательство теоремы заключается в том, чтобы переформулировать задачу оптимального управления (7.1)-(7.3) в абстрактном виде (2.1) и применить к ней теорему 2.1 с последующей расшифровкой. А именно, в силу стандартных теорем существования и единственности для произвольных $\left(x_{1}, u(\cdot)\right) \in X$, достаточно близких к $\left(x_{0}\left(t_{1}\right), u_{0}(\cdot)\right)$, существует и единственно решение задачи Коши

$$
\dot{x}=f(x, u(t), t), \quad x\left(t_{1}\right)=x_{1}, \quad t \in\left[t_{1}, t_{2}\right],
$$

которое обозначим через $x(\cdot)$. Для указанных $\left(x_{1}, u(\cdot)\right)$ положим

$$
\begin{gathered}
F_{s}\left(x_{1}, u(\cdot)\right)=K_{s}\left(x_{1}, x\left(t_{2}\right)\right), \quad s=1,2 ; \\
f_{0}\left(x_{1}, u(\cdot)\right)=K_{0}\left(x_{1}, x\left(t_{2}\right)\right)+\int_{t_{1}}^{t_{2}} f^{0}(x(t), u(t), t) d t .
\end{gathered}
$$

Рассмотрим задачу

$$
f_{0}\left(x_{1}, u(\cdot)\right) \rightarrow \min , \quad F_{1}\left(x_{1}, u(\cdot)\right) \leqslant 0, \quad F_{2}\left(x_{1}, u(\cdot)\right)=0 .
$$

Точка $\left(x_{0}\left(t_{1}\right), u_{0}(\cdot)\right)$ является в ней минимумом, локальным относительно конечной топологии. Поэтому к ней применимы необходимые условия первого и второго порядка теоремы 2.1. При этом принцип Лагранжа (2.2) после стандартных преобразований (подробности см. в [3; §4.1]) дает условия Эйлера-Лагранжа, а (2.4), после таких же преобразований, дает (7.12). При этом используется, что коразмерность подпространства $\mathscr{N}_{K} \subseteq X$ равна $d(K)-d$ (см. предложение 1 из [42]) и $\mathscr{N}_{K}$ совпадает с ядром производной отображения $F=\left(F_{1}, F_{2}\right): X \rightarrow \mathbb{R}^{d(K)}$ в точке $\left(x_{0}\left(t_{1}\right), u_{0}(\cdot)\right)$.

Обсудим теорему. Как известно, для экстремали $\left(x_{0}(\cdot), u_{0}(\cdot)\right)$ условие $d=0$ равносильно управляемости системы уравнений в вариациях (7.8) (условие нормальности), из которой вытекает локальная управляемость системы (7.1) в окрестности $\left(x_{0}(\cdot), u_{0}(\cdot)\right)$. Если хотя бы один из концов траектории $x_{1}$ или $x_{2}$ свободен (т. е. отображение $K$ от соответствующей переменной $x_{1}$ или $x_{2}$ не зависит), то $d=0$. Если же хотя бы один из концов траектории $x_{1}$ или $x_{2}$ заранее фиксирован, то можно взять $\theta=\min (d, n)$. 
Пусть концевые ограничения типа неравенств отсутствуют (т. е. $\left.d\left(K_{1}\right)=0\right)$. Тогда предположение (7.11) в формулировке теоремы можно опустить. Если при этом $d=0$, то $\Lambda\left(x_{0}(\cdot), u_{0}(\cdot)\right)$ состоит из единственной точки $\lambda$ и теорема гарантирует неотрицательность формы $\Gamma(\lambda)$ на $\mathscr{K}$, а это классические необходимые условия второго порядка. Если же экстремаль, являющаяся слабым минимумом, анормальна, (т. е. $d \neq 0)$, то может не существовать такого $\lambda \in \Lambda\left(x_{0}(\cdot), u_{0}(\cdot)\right)$, что форма $\Gamma(\lambda)$ неотрицательна на $\mathscr{K}$. Соответствующий пример был предложен Э. Дж. Макшейном еще в 1941 г. (см. [11]).

Задачи со смешанными ограничениями. Рассмотрим задачу оптимального управления со смешанными ограничениями

$$
R_{1}(x, u, t) \leqslant 0, \quad R_{2}(x, u, t)=0 .
$$

Здесь $R_{s}: \mathbb{R}^{n} \times \mathbb{R}^{m} \times \mathbb{R} \rightarrow \mathbb{R}^{d\left(R_{s}\right)}, s=1,2$, - заданные отображения, удовлетворяющие тем же предположениям гладкости, что и $f$. В этой задаче допустимым процессом называется пара вектор-функций $(x(t), u(t)), t \in\left[t_{1}, t_{2}\right]$, в которой $u(\cdot)$ - допустимое управление, а соответствующее ему решение $x(\cdot)$ уравнения (7.1) удовлетворяет концевым ограничениям (7.2) и смешанным ограничениям $R_{1}(x(t), u(t), t) \leqslant 0, R_{2}(x(t), u(t), t)=0 \dot{\forall} t$.

Рассмотрим допустимый процесс $\left(x_{0}(\cdot), u_{0}(\cdot)\right)$. Будем предполагать, что смешанные ограничения регулярны вдоль этого процесса, т. е. существует $\varepsilon_{0}>0$ такое, что для почти всех $t \in\left[t_{1}, t_{2}\right]$ у матрицы $\frac{\partial R}{\partial u}(t)$ найдется минор порядка $\left|I_{\varepsilon_{0}}(t)\right|$, стоящий в последних $d\left(R_{2}\right)$ столбцах, а также в столбцах с номерами $i \in I_{\varepsilon_{0}}(t)$, модуль которого не меньше $\varepsilon_{0}$. Здесь $R=\left(R_{1}, R_{2}\right),|I|$ - количество элементов множества $I$ и для $\varepsilon \geqslant 0$

$$
I_{\varepsilon}(t):=\left\{i: 1 \leqslant i \leqslant d\left(R_{1}\right), R_{1, i}(t) \geqslant-\varepsilon\right\},
$$

а также используются обозначения

$$
R_{1}(t):=R_{1}\left(x_{0}(t), u_{0}(t), t\right), \quad \frac{\partial R}{\partial u}(t):=\frac{\partial R}{\partial u}\left(x_{0}(t), u_{0}(t), t\right) \quad \text { и т. д. }
$$

Будем говорить, что допустимый процесс $\left(x_{0}(\cdot), u_{0}(\cdot)\right)$ удовлетворяет уравнению Эйлера-Лагранжа в задаче со смешанными ограничениями, если существуют вектор $\lambda$, удовлетворяющий (7.4), и вектор-функция

$$
\begin{gathered}
\eta=\left(\eta_{1}, \eta_{2}\right): \quad \eta_{s} \in L_{\infty}^{d\left(R_{s}\right)}\left[t_{1}, t_{2}\right], \quad s=1,2 ; \\
\eta_{1}(t) \geqslant 0, \quad\left\langle\eta_{1}(t), R_{1}(t)\right\rangle=0 \quad \dot{\forall} t
\end{gathered}
$$

такие, что вектор-функция $\psi$, являющаяся решением задачи Коши

$$
\dot{\psi}=-\frac{\partial H}{\partial x}\left(x_{0}(t), u_{0}(t), t, \psi, \lambda^{0}\right)+\eta(t) \frac{\partial R}{\partial x}(t)^{*}, \quad \psi\left(t_{1}\right)=\frac{\partial l}{\partial x_{1}}\left(p_{0}, \lambda\right),
$$

удовлетворяет условиям (7.6) и

$$
\frac{\partial H}{\partial u}\left(x_{0}(t), u_{0}(t), t, \psi(t), \lambda^{0}\right)-\eta(t) \frac{\partial R}{\partial u}(t)^{*}=0 \quad \dot{\forall} t .
$$


Используя регулярность смешанных ограничений и неравенство Гронуолла, получаем, что каждому вектору $\lambda$ в силу условий Эйлера-Лагранжа отвечает единственная пара $(\psi(\cdot), \eta(\cdot))$. Через $\Lambda_{R}\left(x_{0}(\cdot), u_{0}(\cdot)\right)$ обозначим множество таких $\lambda$, что пара $(\lambda, \eta)$ для некоторой вектор-функции $\eta$ отвечает процессу $\left(x_{0}(\cdot), u_{0}(\cdot)\right)$ в силу уравнения Эйлера-Лагранжа в задаче со смешанными ограничениями.

Через $\mathscr{K}_{R}$ обозначим множество пар $(\xi, \delta u) \in X$, удовлетворяющих условиям (7.9) и

$$
\begin{array}{cl}
\left\langle\delta x(t), \frac{\partial R_{1, i}}{\partial x}(t)\right\rangle+\left\langle\delta u(t), \frac{\partial R_{1, i}}{\partial u}(t)\right\rangle \leqslant 0 & \forall i \in I_{0}(t), \\
\delta x(t) \frac{\partial R_{2}}{\partial x}(t)+\delta u(t) \frac{\partial R_{2}}{\partial u}(t)=0 & \dot{\forall} t .
\end{array}
$$

В $X$ рассмотрим подпространство $\mathscr{N}_{R}$, состоящее из всех $(\xi, \delta u)$, для которых

$$
\begin{array}{cl}
\left\langle\delta x(t), \frac{\partial R_{1, i}}{\partial x}(t)\right\rangle+\left\langle\delta u(t), \frac{\partial R_{1, i}}{\partial u}(t)\right\rangle=0 & \forall i \in I_{0}(t), \\
\delta x(t) \frac{\partial R_{2}}{\partial x}(t)+\delta u(t) \frac{\partial R_{2}}{\partial u}(t)=0 & \dot{\forall} t .
\end{array}
$$

Для $\lambda \in \Lambda_{R}\left(x_{0}(\cdot), u_{0}(\cdot)\right)$ на $X$ определим квадратичную форму $\Gamma_{R}(\lambda)$ :

$$
\begin{aligned}
\Gamma_{R}(\xi, \delta u ; \lambda) & =\Gamma(\xi, \delta u ; \lambda) \\
& +\int_{t_{1}}^{t_{2}}\left\langle\eta(t), \frac{\partial^{2} R}{\partial(x, u)^{2}}\left(x_{0}(t), u_{0}(t), t\right)[(\delta x(t), \delta u(t)),(\delta x(t), \delta u(t))]\right\rangle d t .
\end{aligned}
$$

Через $\Lambda_{\theta_{R}}=\Lambda_{\theta_{R}}\left(x_{0}(\cdot), u_{0}(\cdot)\right)$ обозначим множество тех $\lambda \in \Lambda_{R}\left(x_{0}(\cdot), u_{0}(\cdot)\right)$, для которых индекс сужения формы $\Gamma_{R}(\lambda)$ на подпространство $\mathscr{N}_{K} \cap \mathscr{N}_{R}$ не превышает числа $\theta_{R}=\min \left(d_{R}, 2 n\right)$. Здесь $d_{R}$ размерность ядра расширенной матрицы управляемости $D_{R}$, явный вид которой приведен в [42]. В частном случае, когда отображение $R$ от переменной $x$ не зависит, $D_{R}$ вычисляется по формуле (7.10), в которой матрица $B(t)$ заменена на $B_{R}(t)=P(t) B(t)$. Здесь $P(t)$ - оператор ортогонального проектирования $\mathbb{R}^{m}$ на подпространство

$$
\left\{u \in \mathbb{R}^{m}:\left\langle\frac{\partial R_{1, i}}{\partial u}(t), u\right\rangle=0 \forall i \in I_{0}(t), u \frac{\partial R_{2}}{\partial u}(t)=0\right\} .
$$

ТеОРема 7.2. Пусть допустимый процесс $\left(x_{0}(\cdot), u_{0}(\cdot)\right)$ является слабым минимумом в задаче со смешанными ограничениями (7.1)-(7.3), (7.14). Тогда $\Lambda_{\theta_{R}} \neq \varnothing$ и для произвольных $(\xi, \delta u) \in \mathscr{K}_{R}$, удовлетворяющих (7.11), имеет место неравенство

$$
\max _{\lambda \in \Lambda_{\theta_{R}}} \Gamma_{R}(\xi, \delta u ; \lambda) \geqslant 0
$$

Вот суть доказательства теоремы 7.2. Вначале, вводя дополнительное управление $v \in \mathbb{R}^{d\left(R_{1}\right)}$, рассмотрим задачу (7.1)-(7.3) со смешанными ограничениями

$$
R_{1}(x, u, t)-v=0, \quad R_{2}(x, u, t)=0, \quad v \leqslant 0,
$$


которая, очевидно, эквивалентна исходной задаче (7.1)-(7.3), (7.14). Используя регулярность смешанных ограничений (7.14), разрешая локально систему уравнений $R_{1}(x, u, t)-v=0, R_{2}(x, u, t)=0$ относительно части переменных, сведем исходную задачу к задаче, в которой вместо смешанных ограничений имеются лишь геометрические ограничения на управления $v \leqslant 0$ (подробности см. в [42]).

Переформулировав рассматриваемую задачу как задачу минимизации в банаховом пространстве $\mathbb{R}^{n} \times L_{\infty}^{m}\left[t_{1}, t_{2}\right]$, получаем задачу $(7.13)$, но с дополнительным ограничением на управление: $v(\cdot) \in C$, где $C=\left\{v(\cdot) \in L_{\infty}^{d\left(R_{1}\right)}: v(t) \leqslant 0\right.$ $\dot{\forall} t\}$. Применяя к этой задаче теорему 2.4 (подробности см. в [43]), получаем, что $\Lambda_{\theta_{R}} \neq \varnothing$, и для произвольных $(\xi, \delta u) \in \widetilde{\mathscr{K}}$ имеет место (7.15). Здесь $\widetilde{\mathscr{K}}$ состоит из тех $(\xi, \delta u) \in X$, для которых выполняются условия (7.9), (7.11) и $\delta v \in C+\operatorname{span}\left\{v_{0}\right\}$, где $\delta v$ и $v_{0}-$ первые $d\left(R_{1}\right)$ координат вектор-функций $\delta u$ и $u_{0}$ соответственно. Но по теореме 2 из [12] замыкание множества $\widetilde{\mathscr{K}}$ совпадает с множеством тех $(\xi, \delta u) \in \mathscr{K}_{R}$, для которых выполняется (7.11). Кроме того, как отмечалось в разделе 2 (см. также лемму 1 из [12]), множество $\Lambda_{\theta_{R}}$ компактно и, значит, функция максимума $\gamma(\xi, \delta u)=\max _{\lambda \in \Lambda_{\theta_{R}}} \Gamma(\xi, \delta u ; \lambda)$ непрерывна. Искомое утверждение вытекает из неотрицательности функции $\gamma$ на $\widetilde{\mathscr{K}}$.

В задачах оптимального управления с фазовыми ограничениями и с нефиксированным временем необходимые условия второго порядка, заключающиеся в непустоте множества множителей Лагранжа $\Lambda_{s}$, при соответствующем выборе числа $s$ были получены в [9]-[11]. В указанных работах необходимые условия первого порядка брались в форме принципа максимума Понтрягина, а не уравнения Эйлера-Лагранжа.

\section{8. Некоторые приложения}

Теория бифуркаций. Пусть $X, \Sigma, Y$ - банаховы пространства и задано отображение $F: X \times \Sigma \rightarrow Y$, которое дважды непрерывно дифференцируемо в окрестности точки $\left(x_{0}, \sigma_{0}\right)$. Будем предполагать, что подпространство ker $\frac{\partial F}{\partial x}\left(x_{0}, \sigma_{0}\right)$ топологически дополняемо, а коразмерность подпространства $\operatorname{im} \frac{\partial F}{\partial x}\left(x_{0}, \sigma_{0}\right)$ конечна (тогда оно замкнуто). Пусть $F\left(x_{0}, \sigma\right)=0$ для всех $\sigma$ из некоторой окрестности точки $\sigma_{0}$.

Напомним, что точка $\left(x_{0}, \sigma_{0}\right)$ называется точкой бифуркации, если существует последовательность $\left\{\left(x_{i}, \sigma_{i}\right)\right\}, x_{i} \neq 0 \forall i$, которая сходится к $\left(x_{0}, \sigma_{0}\right)$, и $F\left(x_{i}, \sigma_{i}\right)=0 \forall i$. Если точка $x_{0}$ нормальна, т. е. $\operatorname{im} \frac{\partial F}{\partial x}\left(x_{0}, \sigma_{0}\right)=Y$, то бифуркации описываются с помощью ядра $\operatorname{ker} \frac{\partial F}{\partial x}\left(x_{0}, \sigma_{0}\right)$. Особый интерес представляют точки бифуркации $\left(x_{0}, \sigma_{0}\right)$, когда $x_{0}$ анормальна.

С помощью результатов раздела 2 можно получить достаточные условия бифуркации и в анормальном случае. Рассмотрим описанную выше точку $\left(x_{0}, \sigma_{0}\right)$. Вначале с помощью процедуры Ляпунова-Шмидта сведем задачу 
к случаю, когда $Y=\mathbb{R}^{k}, \frac{\partial F}{\partial x}\left(x_{0}, \sigma_{0}\right)=0$. Затем рассмотрим задачу минимизации

$$
f_{0}(x, \sigma):=\left\langle x-x_{0}, x^{*}\right\rangle \rightarrow \min , \quad F(x, \sigma)=0,
$$

где $x^{*}$ - произвольный ненулевой линейный непрерывный функционал на $X$. Ясно, что если точка $\left(x_{0}, \sigma_{0}\right)$ не является локальным минимумом рассматриваемой задачи, то она является точкой бифуркации. Поэтому, применяя теорему 2.7 (точнее, выписывая отрицание условия (2.18)), получаем достаточные условия бифуркации (см. [44]).

Теория чувствительности. В приложениях нередко приходится исследовать не одну экстремальную задачу, а целое их семейство, например,

$$
f_{0}(x, \sigma) \rightarrow \min , \quad F(x, \sigma)=0,
$$

где $\sigma \in \Sigma$ - параметр. Спрашивается, как зависит от $\sigma$ точка минимума рассматриваемой задачи, или хотя бы значение этого минимума $\omega(\sigma)$, и даже будет ли непусто множество допустимых точек $\{x: F(x, \sigma)=0\}$ при всех $\sigma$, близких к номинальному значению параметра $\sigma_{0}$, при котором решение $x_{0}$ известно. Например, непрерывность функции минимума $\omega$ означает корректность задачи. Если точка $x_{0}$ нормальна, то эти вопросы хорошо изучены с помощью классической теоремы о неявной функции (см. [14] и библиографию там). Однако, если точка $x_{0}$ анормальна, то эта техника уже неприменима. Но и в этом случае с помощью теорем о неявной функции из раздела 6 и близких к ним утверждений в [45] исследована полунепрерывность сверху и снизу функции $\omega$, для нее получены оценки сверху, снизу и асимптотические разложения. В [46] эти результаты обобщены на задачу с ограничениями $F(x, \sigma) \in C$, где $C$ выпуклый замкнутый конус.

Теория управляемости. Рассмотрим управляемую систему (7.1) с начальным условием $x\left(t_{1}\right)=x_{1}$, где $x_{1}$ задано. Пусть задан допустимый процесс $\left(x_{0}(\cdot), u_{0}(\cdot)\right)$. Спрашивается, является ли он локально управляемым, т. е. можно ли с помощью допустимых управлений, достаточно близких к $u_{0}(\cdot)$, попасть по траектории управляемой системы (7.1) в момент времени $t_{2}$ в произвольную точку, лежащую в некоторой окрестности точки $x_{0}\left(t_{2}\right)$. Если для рассматриваемого процесса линейная система уравнений в вариациях (7.8) управляема, т. е. ядро расширенной матрицы управляемости (7.10) является нулевым, то утвердительный ответ на этот вопрос дает классическая теорема об обратной функции. Если же это ядро ненулевое, т. е. процесс $\left(x_{0}(\cdot), u_{0}(\cdot)\right)$ является анормальным, то для исследования локальной управляемости классическая теорема об обратной функции уже неприменима. Но и в этом случае теоремы о неявной и обратной функции из раздела 6 дают достаточные условия локальной управляемости (см. [47]).

Теория квадратичных отображений. В недавней работе [48] среди других сформулированы две открытые проблемы теории квадратичных отображений. Первая из них: для заданного квадратичного отображения $Q=$ $\left(q_{1}, \ldots, q_{k}\right): \quad X=\mathbb{R}^{n} \rightarrow \mathbb{R}^{k}$ найти достаточные условия, сформулированные 
в терминах матриц $Q_{i}$, при которых множество его нетривиальных нулей $K=$ $\{x: Q(x)=0, x \neq 0\}$ пусто. Вторая проблема заключается в нахождении достаточных условий, при которых

$$
\begin{gathered}
\max \left\{q_{1}(x), \ldots, q_{k}(x)\right\}>0 \quad \forall x \neq 0 \\
\left(\text { соответственно } \max \left\{q_{1}(x), \ldots, q_{k}(x)\right\} \geqslant 0 \forall x\right) .
\end{gathered}
$$

Решение этих задач основано на результатах раздела 5 . Для целых $s \geqslant 0$ положим

$$
\begin{gathered}
\mathscr{Y}_{s}^{+}=\left\{y^{*} \neq 0: \text { ind }^{+} y^{*} Q \leqslant s\right\}, \quad \widetilde{\mathscr{Y}}_{s}=\left\{y^{*} \in \mathscr{Y}_{s}: y^{*} \geqslant 0\right\}, \\
\widetilde{\mathscr{Y}}_{s}^{+}=\left\{y^{*} \in \mathscr{Y}_{s}^{+}: y^{*} \geqslant 0\right\} .
\end{gathered}
$$

Здесь ind $^{+} q$ - неотрицательный индекс квадратичной формы $q$, т. е. количество неположительных собственных значений матрицы этой формы. Отметим, что, в отличие от конуса $\mathscr{Y}_{s}$, пересечение конуса $\mathscr{Y}_{s}^{+}$с единичной сферой может оказаться незамкнуто.

Tеорема 8.1. Условие $K=\varnothing$ эквивалентно тому, что

$$
\forall x \in X \backslash\{0\} \quad \exists y^{*} \in \mathscr{Y}_{k-1}^{+}:\left\langle y^{*}, Q(x)\right\rangle>0 .
$$

Если выполняется (5.19), то условие $K=\varnothing$ эквивалентно тому, что

$$
\forall x \in X \backslash\{0\} \quad \exists y^{*} \in \mathscr{Y}_{k-2}^{+}:\left\langle y^{*}, Q(x)\right\rangle>0 .
$$

Теорема 8.2. Первое условие из (8.1) эквивалентно тому, что

$$
\forall x \in X \backslash\{0\} \quad \exists y^{*} \in \widetilde{\mathscr{Y}}_{k-2}^{+}:\left\langle y^{*}, Q(x)\right\rangle>0 .
$$

Второе условие из (8.1) эквивалентно тому, что

$$
\forall x \in X \quad \exists y^{*} \in \widetilde{\mathscr{Y}}_{k-2}:\left\langle y^{*}, Q(x)\right\rangle \geqslant 0 .
$$

Итак, теорема 8.1 дает решение первой задачи, а теорема 8.2 - второй. Доказательство теоремы 8.1 основано на теореме 5.2, а теорема 8.2 выводится из теоремы 8.1.

\section{Список литературы}

[1] А. Д. Иоффе, В. М. Тихомиров, Теория экстремальных задач, Наука, М., 1974, 479 с.; англ. пер.: A. D. Ioffe, V. M. Tihomirov, Theory of extremal problems, Stud. Math. Appl., 6, North-Holland, Amsterdam-New York, 1979, xii+460 pp.

[2] A. L. Dontchev, R. T. Rockafellar, Implicit functions and solution mappings. A view from variational analysis, Springer Monogr. Math., Springer, Dordrecht, 2009, xii+375 pp.

[3] В. М. Алексеев, В. М. Тихомиров, С. В. Фомин, Оптимальное управление, Наука, M., 1979, 430 с.; англ. пер.: V. M. Alekseev, V. M. Tikhomirov, S. V. Fomin, Optimal control, Contemp. Soviet Math., Consultants Bureau, New York, 1987, xiv+309 pp. 
[4] А.В. Арутюнов, "Условия второго порядка в экстремальных задачах с конечномерным образом. 2-нормальные отображения", Изв. РАН. Сер. матем., 60:1 (1996), 37-62; англ. пер.: A. V. Arutyunov, "Second-order conditions in extremal problems with finite-dimensional range. 2-normal maps", Izv. Math., 60:1 (1996), 39-65.

[5] А.В. Арутюнов, Условия экстремума. Анормалъные и вырожденные задачи, Изд-во "Факториал", М., 1997, 255 с.; см. также: A. V. Arutyunov, Optimality conditions: Abnormal and degenerate problems, Math. Appl., 526, Kluwer, Dordrecht, 2000, x+299 pp.

[6] А. В. Арутюнов, "Необходимые условия экстремума и теорема об обратной функции без априорных предположений нормальности", Дифференииальные уравнения и динамические системы, Сборник статей. K 80-летию со дня рождения академика Евгения Фроловича Мищенко, Тр. МИАН, 236, Наука, М., 2002, 33-44; англ. пер.: А. V. Arutyunov, "Necessary conditions for an extremum and an inverse function theorem without a priori normality assumptions", Proc. Steklov Inst. Math., 236 (2002), 25-36.

[7] Е. С. Левитин, А.А. Милютин, Н.П. Осмоловский, "Условия высших порядков локального минимума в задачах с ограничениями", УМН, 33:6(204) (1978), 85-148; англ. пер.: E. S. Levitin, А. A. Milyutin, N. P. Osmolovskii, "Conditions of high order for a local minimum in problems with constraints", Russian Math. Surveys, 33:6 (1978), 97-168.

[8] А. А. Милютин, "О квадратичных условиях экстремума в гладких задачах с конечномерным образом", Методы теории экстремальных задач в экономике, Наука, М., 1981, 137-177.

[9] А.В. Арутюнов, Н. Т. Тынянский, "К необходимым условиям локального минимума в теории оптимального управления", Докл. АН CCCP, 275:2 (1984), 268-272; англ. пер.: А. V. Arutyunov, N. T. Tynyanskŭ, "On necessary conditions for a local minimum in optimal control theory", Soviet Math. Dokl., 29:2 (1984), 176-179.

[10] А.В. Арутюнов, "K необходимым условиям оптимальности в задаче с фазовыми ограничениями", Докл. АН СССР, 280:5 (1985), 1033-1037; англ. пер.: A.V. Arutyunov, "On necessary optimality conditions in a problem with phase constraints", Soviet Math. Dokl., 31:1-6 (1985), 174-177.

[11] А.В. Арутюнов, "Возмущения экстремальных задач с ограничениями и необходимые условия оптимальности", Итоги науки и техники. Матем. анализ., 27, ВИНИТИ, М., 1989, 147-235; англ. пер.: А. V. Arutyunov, "Perturbations of extremal problems with constraints, and necessary conditions for optimality", J. Soviet Math., 54:6 (1991), 1342-1400.

[12] А. В. Арутюнов, Н. Ю. Черникова, "Свойства конуса критических направлений в задачах оптимального управления", Вестн. Моск. ун-та. Сер. XV. Вычисл. матем. Кибернет., 2006, № 2, 15-19; англ. пер.: А. V. Arutyunov, N. Yu. Chernikova, "Critical cone properties in optimal control problems", Moscow Univ. Comput. Math. Cybernet., 2006, № 2, 13-18.

[13] A. Arutyunov, F. L. Pereira, "Second-order necessary optimality conditions for problems without a priori normality assumptions", Math. Oper. Res., 31:1 (2006), 1-12.

[14] J.F. Bonnans, A. Shapiro, Perturbation analysis of optimization problems, Springer Ser. Oper. Res., Springer-Verlag, New York, 2000, xviii+601 pp.

[15] J. F. Bonnans, R. Cominetti, A. Shapiro, "Second order optimality conditions based on parabolic second order tangent sets", SIAM J. Optim., 9:2 (1999), 466-492.

[16] S. M. Robinson, "Stability theory for systems of inequalities. Part II: Differentiable nonlinear systems", SIAM J. Numer. Anal., 13:4 (1976), 497-513. 
[17] А. В. Арутюнов, Д. Ю. Карамзин, "Необходимые условия минимума в анормальных задачах с геометрическими ограничениями", Журн. вычисл. матем. и матем. физ., 47:3 (2007), 364-375; англ. пер.: A. V. Arutyunov, D. Yu. Karamzin, "Necessary optimality conditions for abnormal problems with geometric constraints", Comput. Math. Math. Phys., 47:3 (2007), 349-360.

[18] А.В. Арутюнов, Д. Ю. Карамзин, "Необходимые условия экстремума в анормальной задаче с ограничениями типа равенств", Журн. вычисл. матем. и матем. физ., 46:8 (2006), 1363-1368; англ. пер.: A. V. Arutyunov, D. Yu. Karamzin, "Necessary optimality conditions in an abnormal optimization problem with equality constraints", Comput. Math. Math. Phys., 46:8 (2006), 1293-1298.

[19] М. Хирш, Дифференциальная топология, Мир, М., 1979, 279 с.; пер. с англ.: M. W. Hirsch, Differential topology, Grad. Texts Math., 33, Springer-Verlag, New York-Heidelberg, 1976, x+221 pp.

[20] Е.Р. Аваков, "Условия экстремума для гладких задач с ограничениями типа равенств", Журн. вычисл. матем. и матем. физ., 25:5 (1985), 680-693; англ. пер.: E. R. Avakov, "Extremum conditions for smooth problems with equality-type constraints", U.S.S.R. Comput. Math. Math. Phys., 25:3 (1985), 24-32.

[21] A. Arutyunov, E. Avakov, B. Gel'man, A. Dmitruk, V. Obukhovskii, "Locally covering maps in metric spaces and coincidence points", J. Fixed Point Theory Appl., 5:1 (2009), 106-127.

[22] Е. Р. Аваков, А. В. Арутюнов, “Теорема об обратной функции и условия экстремума для анормальных задач с незамкнутым образом", Матем. сб., 196:9 (2005), 3-22; англ. пер.: E. R. Avakov, A. V. Arutyunov, "Inverse function theorem and conditions of extremum for abnormal problems with non-closed range", Sb. Math., 196:9 (2005), 1251-1269.

[23] A. V. Arutyunov, E. R. Avakov, A. F. Izmailov, "Necessary optimality conditions for constrained optimization problems under relaxed constraint qualifications", Math. Program., 114:1, Ser. A (2008), 37-68.

[24] А. В. Арутюнов, Д. Ю. Карамзин, "Регулярные нули квадратичных отображений и их приложение", Матем. сб., 202:6 (2011), 3-28; англ. пер.: А. V. Arutyunov, D. Yu. Karamzin, "Regular zeros of quadratic maps and their application", Sb. Math., 202:6 (2011), 783-806.

[25] М.Ф. Сухинин, "Полутейлоровские снизу отображения и достаточные условия экстремума", Матем. сб., 182:6 (1991), 877-891; англ. пер.: M. F. Sukhinin, "Lower semi-Taylor mappings and sufficient conditions for an extremum", Math. USSR-Sb., 73:1 (1992), 257-271.

[26] Р. Беллман, Введение в теорию матрии, Наука, М., 1969, 367 с.; пер. с англ.: R. Bellman, Introduction to matrix analysis, McGraw-Hill, New York-Toronto-London, 1960, xx+328 pp.

[27] А.В. Арутюнов, "О свойствах квадратичных отображений в банаховом пространстве", Матем. заметки, 50:4 (1991), 10-20; англ. пер.: А. V. Arutyunov, "Properties of quadratic maps in a Banach space", Math. Notes, 50:4 (1991), 993-999.

[28] Е. Р. Аваков, А. А. Аграчев, А. В. Арутюнов, "Множество уровня гладкого отображения в окрестности особой точки и нули квадратичного отображения", $M a$ тем. сб., 182:8 (1991), 1091-1104; англ. пер.: E. R. Avakov, A. A. Agrachev, A. V. Arutyunov, "The level set of a smooth mapping in a neighborhood of a singular point, and zeros of a quadratic mapping", Math. USSR-Sb., 73:2 (1992), 455-466.

[29] L. L. Dines, "On the mapping of quadratic forms", Bull. Amer. Math. Soc., 47 (1941), 494-498.

[30] А.А. Аграчев, “Топология квадратичных отображений и гессианы гладких отображений”, Итоги науки и техники. Алгебра. Топол. Геом., 26, ВИНИТИ, М., 1988, 
85-124; англ. пер.: А. А. Agrachev, "Topology of quadratic maps and Hessians of smooth maps", J. Soviet Math., 49:3 (1990), 990-1013.

[31] А.С. Матвеев, "О выпуклости образов квадратичных отображений”, Алгебра и анализ, 10:2 (1998), 159-196; англ. пер.: А. S. Matveev, "On the convexity of the images of quadratic mappings", St. Petersburg Math. J., 10:2 (1999), 343-372.

[32] А. В. Арутюнов, "Неотрицательность квадратичных форм на пересечении квадрик и квадратичные отображения", Матем. заметки, 84:2 (2008), 163-174; англ. пер.: A. V. Arutyunov, "Nonnegativity of quadratic forms on intersections of quadrics and quadratic maps", Math. Notes, 84:1-2 (2008), 155-165.

[33] А.В. Арутюнов, "Положительность квадратичных форм на пересечении квадрик", Матем. заметки, 71:1 (2002), 27-36; англ. пер.: А. V. Arutyunov, "Positive quadratic forms on intersections of quadrics", Math. Notes, 71:1 (2002), 25-33.

[34] А.В. Арутюнов, "О вещественных квадратичных формах, аннулирующих пересечение квадрик", УМН, 60:1(361) (2005), 161-162; англ. пер.: А. V. Arutyunov, "On real quadratic forms annihilating an intersection of quadrics", Russian Math. Surveys, 60:1 (2005), 157-158.

[35] А.В. Арутюнов, "Теорема о неявной функции как реализация принципа Лагранжа. Анормальные точки", Матем. сб., 191:1 (2000), 3-26; англ. пер.: A. V. Arutyunov, "Implicit function theorem as a realization of the Lagrange principle. Abnormal points", Sb. Math., 191:1 (2000), 1-24.

[36] А.В. Арутюнов, В. Ячимович, "К теории анормальных экстремальных задач", Вестн. Моск. ун-та. Сер. XV. Вычисл. матем. кибернет., 2000, №1, 34-40; англ. пер.: A. V. Arutyunov, V. Yachimovich, "On extremum theory for abnormal problems", Moscow Univ. Comput. Math. Cybernet., 2000, № 1, 30-37.

[37] А.В. Арутюнов, С.Е. Жуковский, "Существование обратных отображений и их свойства", Дифференииальные уравнения и топология. II, Сборник статей. K 100-летию со дня рождения академика Льва Семеновича Понтрягина, Тр. МИАН, 271, МАИК, М., 2010, 18-28; англ. пер.: А. V. Arutyunov, S. E. Zhukovskiy, "Existence and properties of inverse mappings", Proc. Steklov Inst. Math., 271 (2010), $12-22$.

[38] А. В. Арутюнов, “Теорема о неявной функции без априорных предположений нормальности", Журн. вычисл. матем. и матем. физ., 46:2 (2006), 205-215; англ. пер.: A. V. Arutyunov, "Implicit function theorem without a priori assumptions about normality", Comput. Math. Math. Phys., 46:2 (2006), 195-205.

[39] А.В. Арутюнов, "Накрывание нелинейных отображений на конусе в окрестности анормальной точки", Матем. заметки, 77:4 (2005), 483-497; англ. пер.: A. V. Arutyunov, "Covering of nonlinear maps on a cone in neighborhoods of irregular points", Math. Notes, 77:4 (2005), 447-460.

[40] А.В. Арутюнов, "К теоремам о неявной функции в анормальных точках", Тр. ИММ УрО РАН, 16, 2010, 30-39; англ. пер.: А. V. Arutyunov, "On implicit function theorems at abnormal points", Proc. Inst. Math. Mech., 271:1, suppl. 1 (2010), 18-27.

[41] Е. Р. Аваков, "Теоремы об оценках в окрестности особой точки отображения", Maтем. заметки, 47:5 (1990), 3-13; англ. пер.: E. R. Avakov, "Theorems on estimates in the neighborhood of a singular point of a mapping", Math. Notes, 47:5 (1990), 425-432.

[42] А.В. Арутюнов, Д. Ю. Карамзин, "Необходимые условия слабого минимума в задаче оптимального управления со смешанными ограничениями", Дифферени. уравнения, 41:11 (2005), 1458-1468; англ. пер.: A. V. Arutyunov, D. Yu. Karamzin, "Necessary conditions for a weak minimum in an optimal control problem with mixed constraints", Differ. Equ., 41:11 (2005), 1532-1543.

[43] А.В. Арутюнов, Ю.С. Верещагина, "О необходимых условиях второго порядка в задачах оптимального управления", Дифферени. уравнения, 38:11 (2002), 
1443-1450; англ. пер.: A. V. Arutyunov, Yu.S. Vereshchagina, "On necessary second-order conditions in optimal control problems", Differ. Equ., 38:11 (2002), 1531-1540.

[44] A. V. Arutyunov, A. F. Izmailov, V. Jaćimović, "New bifurcation theorems via the second-order optimality conditions", J. Math. Anal. Appl, 359:2 (2009), 752-764.

[45] А. В. Арутюнов, А. Ф. Измаилов, "Теория чувствительности для анормальных задач оптимизации с ограничениями типа равенств", Журн. вычисл. матем. и матем. физ., 43:2 (2003), 186-202; англ. пер.: A. V. Arutyunov, A. F. Izmailov, "The sensitivity theory for abnormal optimization problems with equality constraints", Comput. Math. Math. Phys., 43:2 (2003), 178-193.

[46] A. V. Arutyunov, A. F. Izmailov, "Sensitivity analysis for cone-constrained optimization problems under the relaxed constraint qualifications", Math. Oper. Res., 30:2 (2005), 333-353.

[47] А.В. Арутюнов, В. Ячимович, “2-нормальные процессы управляемых динамических систем”, Дифферени. уравнения, 38:8 (2002), 1017-1029; англ. пер.: A. V. Arutyunov, V. Jaćimović, "2-normal processes in controlled dynamical systems", Differ. Equ., 38:8 (2002), 1081-1094.

[48] J.-B. Hiriart-Urruty, "Potpourri of conjectures and open questions in nonlinear analysis and optimization", SIAM Rev., 49:2 (2007), 255-273.

А. В. Арутюнов (А. V. Arutyunov)

Российский университет дружбы народов

E-mail: arutun@orc.ru
Поступила в редакцию 14.06.2011 